\title{
Impact of Detector Simulation in Particle Physics Collider Experiments
}

\author{
V. Daniel Elvira ${ }^{a, *}$ \\ ${ }^{a}$ Fermi National Accelerator Laboratory, P. O. Box 500, Batavia, IL 60510-5011, USA
}

\begin{abstract}
Through the last three decades, accurate simulation of the interactions of particles with matter and modeling of detector geometries has proven to be of critical importance to the success of the international high-energy physics (HEP) experimental programs. For example, the detailed detector modeling and accurate physics of the Geant4-based simulation software of the CMS and ATLAS particle physics experiments at the European Center of Nuclear Research (CERN) Large Hadron Collider (LHC) was a determinant factor for these collaborations to deliver physics results of outstanding quality faster than any hadron collider experiment ever before.

This review article highlights the impact of detector simulation on particle physics collider experiments. It presents numerous examples of the use of simulation, from detector design and optimization, through software and computing development and testing, to cases where the use of simulation samples made a difference in the precision of the physics results and publication turnaround, from data-taking to submission. It also presents estimates of the cost and economic impact of simulation in the CMS experiment.

Future experiments will collect orders of magnitude more data with increasingly complex detectors, taxing heavily the performance of simulation and reconstruction software. Consequently, exploring solutions to speed up simulation and reconstruction software to satisfy the growing demand of computing resources in a time of flat budgets is a matter that deserves immediate attention. The article ends with a short discussion on the potential solutions that are being considered, based on leveraging core count growth in multicore machines, using new generation coprocessors, and re-engineering HEP code for concurrency and parallel computing.
\end{abstract}

Keywords: simulation, high energy physics, particle physics, Geant4, software, computing

\footnotetext{
* Corresponding author

Email address: daniel@fnal.gov (V. Daniel Elvira)
} 


\section{Introduction}

Accurate software modeling is essential to design, build and commission the highly sophisticated detectors utilized in experimental particle physics and cosmology. It is also a fundamental tool to analyze and interpret the resulting experimental data.

In particle physics, an "event" is composed of all the data collected in a single occurrence of an experiment. For example, in astroparticle physics an event may be defined as all the data produced by a very energetic cosmic ray particle as it interacts with the atmosphere. In particle colliders, an event includes all the data produced in a beam crossing, when particle interactions occur, with the caveat that some of the collected information may belong to previous crossings due to the finite speed of detector electronics. In neutrino experiments, an event occurs when a neutrino interacts with a nucleus of an atom in the detector. Simulation software in high-energy physics (HEP) experiments is designed to produce, in an ideal scenario, events which are identical to those resulting from the actual experiment. The output data format is typically the same for simulated and real events, so that event processing and physics analysis is performed in the same way and with the same tools.

The typical simulation software package in HEP experiments consists of a chain of modules that starts with a generator of the physics processes of interest. Generators provide the final state particles in a hard collision, a cosmic ray particle shower, a neutrino beam with the desired energy and angular distribution, or any other set of particles to be observed in the detector. A second module simulates the passage of the generated particles through the detector material and its magnetic field. In most contemporary experiments, this detector simulation module is based on the Geant4 simulation toolkit [1, 2], a software package that provides the tools to describe the detector geometry and materials, and incorporates a large number of models to simulate electromagnetic and hadronic interactions of particles with matter. The next module simulates the detector electronics and the calibration of individual channels. At the end of the chain, the same algorithms used to identify and reconstruct individual particles and physics observables in real data are applied to simulated events. Fig. 1 describes a generic simulation software chain and the functionality of each module for a typical HEP experiment.

There was a time, however, when experiments modeled their detectors using simple analytic or back-of-the-envelope calculations, toy simulations, or parametrizations based on theoretical predictions, or experimental data of the passage of particles through matter and electromagnetic fields. The era of detailed detector simulation started in the late 1970's and early 1980's when the Electron Gamma Shower software (EGS) 3] was developed, and the GEANT team released GEANT3 [4, a software toolkit that allowed the experiments to describe complex geometry, propagate particles through these geometries, and trace the incident and secondary particles as they interact with the different materials according to physics models implemented as part of the toolkit. It did not take too long before GEANT3 was widely used at the European Center 


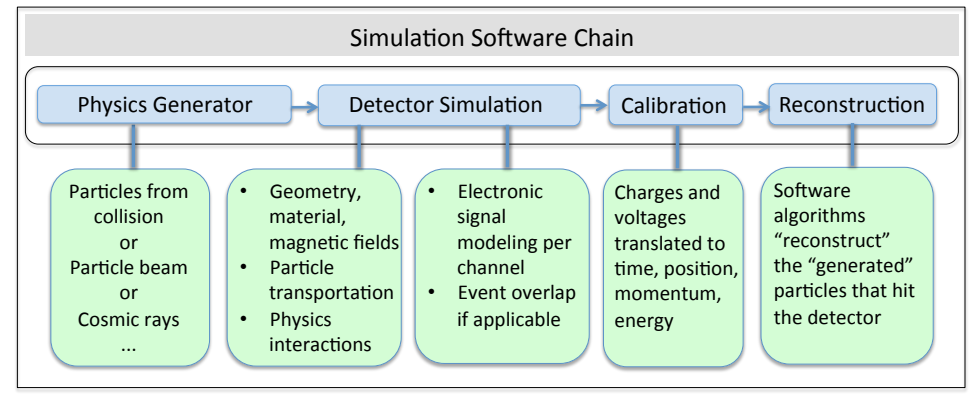

Figure 1: Generic simulation software chain in a typical HEP experiment.

for Nuclear Research (CERN), the German Deutsches Elektronen-Synchrotron (DESY) and the US Fermi National Accelerator Laboratory (FNAL) experiments. Although initially of limited use because of the insufficient speed of computers in those days, these GEANT3-based full detector simulation software applications became the norm through the nineteen nineties and revolutionized the way the particle physics community plans experiments, design detectors, and perform physics measurements.

It should not be necessary to clarify that particles cannot be discovered through simulation. For example, the Higgs boson can only be observed in simulated data if its production mechanisms and decay modes are coded in the event generator package that feeds the detector simulation module. What a simulation does is to teach physicists what mark the Higgs boson would leave in the real detector if it were present in the data sample. For instance, the Standard Model of Elementary Particles and their Interactions (SM) predicts that the Higgs boson decays into two photons with certain kinematic properties. Using simulation, physicists designed detectors and data analysis procedures that targeted Higgs searches with the goal to identify events with the characteristics predicted by theories. In 2012, the observation of events of this kind in real data signaled a discovery by the ATLAS [5] and CMS [6] experiments at the CERN Large Hadron Collider (LHC). Real-life physics measurement procedures are more complex than the example presented here but the general idea is always the same in what pertains to the role of simulation.

During the last three decades, simulation has proven to be of critical importance to the success of HEP experimental programs. For example, the detailed detector modeling and accurate physics of the CMS [7, 8, 9] and ATLAS 10, 11, 12 Geant4-based simulation software was a determinant factor for these experiments to deliver physics results of outstanding quality faster than any hadron collider experiment ever before. Simulation software at the LHC experiments is more accurate and yet runs much faster than their predecessors at the Tevatron, resulting in a faster analysis turnaround and smaller systematic uncertainties in the measurements. As an example, the CMS experiment simulated, reconstructed and stored more than ten billion events during the Run 1, 
2010-2012, data-taking period. This effort required more than half of the total computing resources allocated to the experiment. Simulation samples of better quality and in larger quantities, evolving detector and computing technology, and a wealth of experience from pre-LHC experiments on calibration and data analysis techniques, improved significantly the precision of the measurements in the current generation of experiments and shortened the time between datataking and public results or journal submission.

This review article focuses on collider experiments and places the emphasis on the detector simulation part of the simulation chain, particularly on the Geant4-based module. It explains the concepts of Full and Fast Simulation and the tuning of the many physics models involved. It presents numerous examples where the use of detector simulation made a difference in the precision of the physics measurements and publication turnaround. For this, it borrows heavily but not exclusively from the CMS (LHC) and D0 (Tevatron) experiments, drawing from the author's personal experience. The last two sections include estimates of the cost and economic impact of simulation in HEP and introduce concepts that will shape the detector simulation efforts of the future.

\section{Toy, Parametrized, and Full Simulation}

The common classification of simulation code in "full" and "fast" is misleading since it refers to the speed of the software application, a relative concept, rather than to its nature. Instead, it is more useful to introduce the concepts of Toy Simulation (ToySim), Parametrized Simulation (ParSim), and Full Simulation (FullSim). Often, physicists refer to simulation software and simulated data samples as Monte Carlo (MC) Simulation and MC samples. The expression makes an analogy between randomness in casino games and randomness built in the methods to integrate the equations of motion for particles traversing matter and magnetic fields within the detector. Physics interactions occurring with different probabilities in the detector material, due to quantum mechanics, introduces a second source of randomness.

A Toy Simulation is a basic tool that may consist of a few simple analytical equations. It may be used to demonstrate large physics effects or biases in a measurement as a proof of principle, but it is often not accurate enough to make predictions of the size of these effects or biases. ToySim does not involve a detector geometry description or the detail of particle shower development. Typically, ToySim events take a small fraction of a second to generate.

Most modern Full Simulation applications in HEP are based on Geant4. They include detailed geometry and magnetic fields descriptions and accurate modeling of electromagnetic and hadronic particle showers provided by the many collections of physics models either adopted or developed, as well as optimized and validated by the Geant 4 team. FullSim is the slowest but the option of choice for most studies, as fast and large scale computing became available and made it possible for big experiments to generate tens of billions of complex detector events per year. FullSim of complex HEP detectors typically takes between a few seconds and a few minutes per event to generate. 
A Parametrized Simulation involves a geometry description, parametrizations of the energy response of single particles measured in data or extracted from Full Simulation or theoretical calculations, a mechanism to randomize the results of the parametrizations, and magnetic field maps. The goal is to make the ParSim much faster than the FullSim, typically a couple of orders of magnitude, and almost as accurate. The accuracy limitations of ParSim tools are typically more severe in describing particle shower shapes and related detector effects such as energy leakage beyond the detector boundaries, and regions of phase space where data are not available. ParSim tools may also be based on GEANT3 or Geant4 up-to-the-point of the first interaction of primary incident particles with matter, after which tools such as GFLASH[13] may be used to describe shower shapes and response with better or worse accuracy, depending on the number of parameters used to describe the showers and the time performance cost the experiment is willing to pay for accuracy. GFLASH-based simulations, or a simulation that uses particle shower libraries constructed from FullSim events, are sometimes used in combination with FullSim to model subdetectors with high particle occupancy, for example those located near the beam pipe in collider experiments. ParSim is commonly used for detector design studies that require to test many geometry scenarios, and to generate signal samples for new physics that involve scans over a large sector of theory parameter space. In most cases, the output of ParSim applications has the same format as the output from FullSim and the data coming from the real detector. Instead, an event in a ToySim sample is often a collection of particles with their four-vector position and momentum. ParSim events typically take on the order of a second to a few seconds to generate.

An example of a ParSim is the CDF Fast Simulation software package, also known as QFL, developed in 1989. (In the absence of public documentation, the information about the CDF QFL and GFLASH parametrized simulations was obtained from private communication with Soon Young Jun, Kenichi Hatakeyama, and Marjorie Shapiro.) QFL was based on a detailed geometry description and accurate parametrization of single particle showers. Pion information was extracted from minimum bias and track triggers in the $0.75-20 \mathrm{GeV}$ range and test beam pion data were utilized in the higher $57-145 \mathrm{GeV}$ energy range. This approach provided a suitable solution in the 1989-1990 (Run 0) and 1991-1996 (Run 1) data taking periods to deal with the fact that GEANT3based simulations were still too slow to be practical. In 2002, QFL was replaced with a hybrid application that utilized GEANT3 to model the detector geometry and track particles, but only up to the point when the incident particles interact with the detector material for the first time, with subsequent showers modeled with GFLASH parametrizations tuned to test beam and collider data. In contrast, the D0 experiment followed a FullSim approach from the start of Run 1 in 1991, since the absence of a solenoidal magnetic field within the tracker made it challenging to tune a ParSim tool with high precision. However, in the interest of speed, D0 introduced approximations such as a mixed plate geometry option, based on average material, rather than a full plate option, based on the actual material detail. In addition, showers were truncated once $95 \%$ of the 
energy of the shower was released in the detector material, approximation that had a significant impact on the description of hadron energy response linearity, and on shower shapes. Twenty years later, the availability of more advanced computing and software systems has allowed the ATLAS and CMS experiments to develop FullSim applications and generate tens of billions of Geant4-based events per year with unprecedented geometry, material and magnetic field detail, as well as significantly improved physics models. In CMS, the amount of CPU (Central Processing Unit in a computer) time spent per event during the 2009-2013 (Run 1) period ranged between 15 seconds for the simplest events to three minutes for more complex events such as those with top quarks or many high momentum jets. In contrast, it took up to one hour per event to generate the Monte Carlo sample utilized in the $W$ boson mass measurement at the Tevatron D0 experiment a decade earlier. In the early 1990's, most of the FullSim MC samples used by the Tevatron experiments consisted of a few hundreds of thousands events and included approximations which introduced severe limitations in their utilization.

\section{Simulation Software Tools}

At the core of the impressive agreement between simulation and data at the LHC is Geant4, the detector simulation toolkit developed, maintained, and supported by the Geant4 Collaboration, and currently used by most HEP experiments. The first production version of Geant4, the Object Oriented $\mathrm{C}++$ incarnation of the GEANT family, was released in 1998. Since then, its areas of application have extended to include high-energy, nuclear and accelerator physics, as well as medical science and treatment, and space exploration. The GEANT saga started in 1975 with the release of GEANT1, a very basic framework to drive a simulation program providing a user-defined output with histograms. GEANT2 was released in 1976 as an extension of GEANT1. It had a more complete set of physics models, including electromagnetic (EM) showers based on a subset of the Electron Gamma Shower (EGS) [3] package, multiple scattering, particle decay, and energy loss. GEANT2 was used by several Super Proton Synchrotron (SPS) experiments at CERN. The breakthrough came in 1980 with GEANT3, an evolution of the GEANT software that contained a data structure to describe complex geometries at the level required by the experiments planned for the 1980's. GEANT3 was first used in the OPAL experiment at the CERN Large Electron-Positron Collider (LEP) and then adopted by other LEP experiments, such as L3 and ALEPH. Experiments at DESY and FNAL soon followed suit.

Other simulation tools worth mentioning are FLUKA [14] and MARS [15]. FLUKA is a fully integrated particle physics simulation package with many applications in HEP and engineering, shielding, detector and telescope design, cosmic ray studies, dosimetry, medical physics and radio-biology. MARS is a software package for the simulation of particle transport and interactions with matter in accelerator, detector, spacecraft and shielding components. It is widely used to model radiation shielding enclosures. 
The success of Geant4-based simulation at the LHC was not due to magic, but the result of many years of hard work and partnership between the experiments and the Geant4 Collaboration. It involved a lengthy process to develop, optimize, and validate the many physics models available for use in Geant4 to describe the interaction of particles with the detector material. Different fora, such us meetings of the Geant4 physics groups and dedicated workshops centered on the topic of Geant4 physics validation, served as vehicles of communication, discussion, and information exchange.

\subsection{Geant4 in a Nutshell}

Geant4 is a toolkit because experimenters assemble their simulation package by selecting, implementing and integrating different elements such as geometry (from available Geant4 shapes), materials, magnetic fields, a method of integration of the equation of motion, and a physics list composed of a subset of the many available physics models. These models describe interactions with matter for different types of incident particles with energies as low as $250 \mathrm{eV}$ and as high as $100 \mathrm{TeV}$. Geant4 provides interfaces to communicate with the experiment's software framework, which connects to various services and the other modules in the simulation chain. A detailed description of the Geant4 toolkit may be found elsewhere [1]. In this section, the focus is on the Geant4 physics and its validation.

\subsection{The Physics of Geant4}

The Geant4 simulation tool kit is not only a software engine to propagate particles through a geometric representation of a detector. It comes with a remarkably complete library of physics models to simulate the interactions of particles with matter. Electrons, muons, and charged hadrons interact electromagnetically with matter through processes such as ionization, bremsstrahlung, pair production, and multiple scattering. Examples of photon interactions are the photoelectric, Compton, conversion, and Rayleigh scattering processes. Hadrons, such as pions, kaons, protons, and neutrons are abundantly produced in HEP collisions and interact strongly with nuclei in the detector material. Although QCD is the theory that describes all hadronic interactions, perturbative calculations may be applied only to a small region of phase-space, while hadronization and nucleus interactions are non-perturbative and may only be described by approximate models. A hadronic shower is the result of the interaction of a single hadron with the detector material. It consists of a cascade of strong interactions producing large numbers of secondary particles of diminishing energies. The development of a hadronic shower covers a large range of energy scales, from the hundreds of $\mathrm{GeV}$ down to, in the case of neutrons, thermal energies. Hadronic showers are difficult to model and are of critical importance to simulate events with quark-initiated or gluon-initiated jets in the experiments.

As illustrated in Fig. 2, Geant4 provides a rich inventory of hadronic physics models, typically assembled in physics lists where energy ranges and model-to- 
model transition regions are defined and optimized for different incident particles.

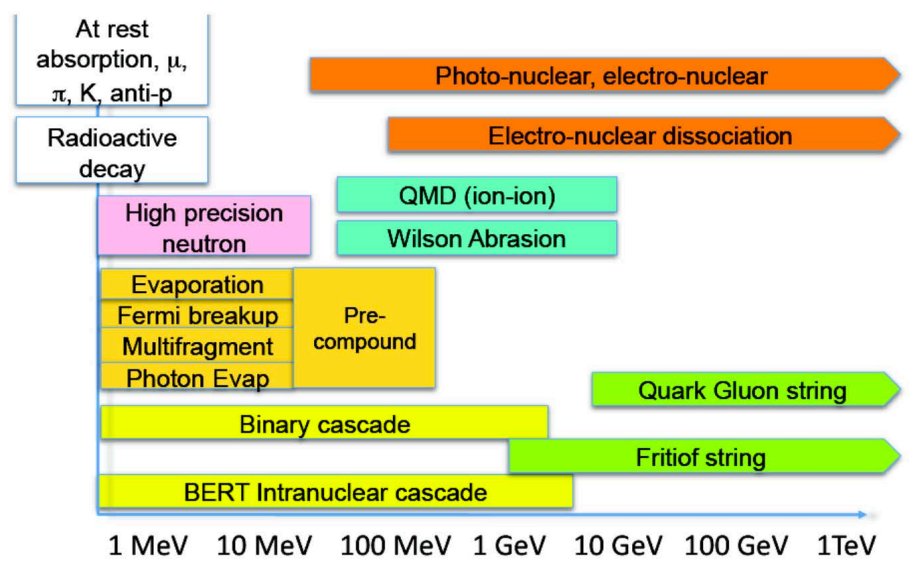

Figure 2: Partial inventory of Geant4 hadronic physics models. "Physics lists" are assembled from a selection of models which are valid in different energy ranges for different particle types.

\subsection{Physics Validation of Geant4}

The task of improving the Geant4 physics models from comparisons between MC predictions and dedicated or thin-target experiments is part of the Geant4 development process. Thin-target experiments consist of directing beams of particles of different types onto thin targets made of the materials typically used in HEP experiments. The measured cross-sections of different nuclear interactions, angular distributions, and particle multiplicities are then used to validate individual models at the microscopic, single-interaction level; examples are the CALICE 16, HARP 17, 18, 19, NA49 20, 21, 22], and NA61 23, 24] experiments. Selecting the physics models to be used in a Geant4 application is not a one-size-fits-all operation, in the sense that some models may represent the data better than others for a given particle type, detector material, and energy range. The reason is that these models typically depend on parameters which are adjusted to the available experimental data, and not all particles, energy ranges, and target materials are present in the currently available thin-target experimental data-sets. Therefore, it is essential for particle physics experiments to validate their Geant4-based simulation software by comparing MC predictions with test beam or collider data. Test beam experiments are designed to study the performance of realistic detector prototypes or solid angle slices of the actual detectors. The data collected are not only essential to understand, optimize and calibrate the detectors, but are also of critical importance to validate the experiment's simulation software. Experiments also contribute to the MC validation process by comparing Geant 4 predictions with in situ measurements 
performed using data collected during their physics runs. Examples of the latter are studies to understand the modeling of single charged tracks, jet response and resolution, and shower shapes.

\subsubsection{Thin-target Experiments}

Figs. 3, 4, 5 illustrate the validation procedure to evaluate the accuracy of the FRITIOF Precompound (FTFP) [25] and Bertini Cascade [26] models in Geant4. The Geant4 FTFP model handles the formation of strings in the hadron-nucleon collision and the subsequent de-excitation of the remnant nucleus. The Geant4 Bertini Model generates the final state for hadron inelastic scattering by simulating the intra-nuclear cascade. This cascade results from the collision of incident hadrons with protons and neutrons in the nucleus of the target material, which produce secondary particles that interact with other nucleons. Fig. 3 shows results for a thin-target experiment with a final state $\pi^{+}$originating from a $158 \mathrm{GeV} / \mathrm{c}$ proton beam that hits a carbon target $\left(\mathrm{p}+\mathrm{C} \rightarrow \pi^{+}+\mathrm{X}\right)$. The observable is the $\pi^{+}$average momentum in the plane transverse to the particle beam, $p_{T}$, as a function of Feynman $x\left(x_{F}\right)$, defined as the ratio between the measured longitudinal momentum of the pion and the maximum value allowed by the kinematics of the collision, $x_{F}=p_{z}^{\pi} / p_{z \max }^{\pi}$. The improvement in the agreement between the Geant4 prediction and the NA49 [20] experimental data is clearly visible, as updates to the FTFP physics model are incorporated to successive Geant4 releases. Fig. 4 shows a Geant4-to-data comparison of the ITEP-771 [27] experiment measurement of the $\pi^{-}(5 \mathrm{GeV})+\mathrm{Cu} \rightarrow \mathrm{n}+\mathrm{X}$ cross section as a function of the neutron kinetic energy. A trend of improvement in the agreement between data and MC is observed for different versions of Geant4, as updates to the Bertini model are incorporated. Fig. 5 shows the polar angle distribution of the outgoing pion with respect to the direction of the incident pion beam versus the momentum of the secondary pion in $\pi^{+}(5 \mathrm{GeV})+\mathrm{Pb} \rightarrow \pi^{+}+\mathrm{X}$ collisions recorded by the HARP experiment [17, 18. The data is compared to a Geant4 prediction, version 10.2.p01, based on the the Bertini model. Figs. 3, 4, 5 are just examples of the many comparison plots available in the Geant4 software validation suite that is used to validate and improve physics models during the development process of a new Geant4 release.

\subsubsection{HEP Experiments}

HEP experiments validate their Geant4-based simulation software using data collected during their physics runs or in dedicated test beam experiments. Fig.6 shows CMS MC-to-data comparison results for isolated charged tracks in minbias events, defined as a beam crossing with the requirement of a hard collision [28. The vertical axis displays the MC-to-data ratio of the ratio of the energy measured in the calorimeters over the momentum measured in the tracker, for a single isolated track. This ratio is measured as a function of the track momentum, $p_{\text {Track }}$. The energy was measured in a $7 \times 7$ cell cluster in the electromagnetic calorimeter $(\mathrm{ECAL})$ and a $3 \times 3$ cell cluster in the hadronic 

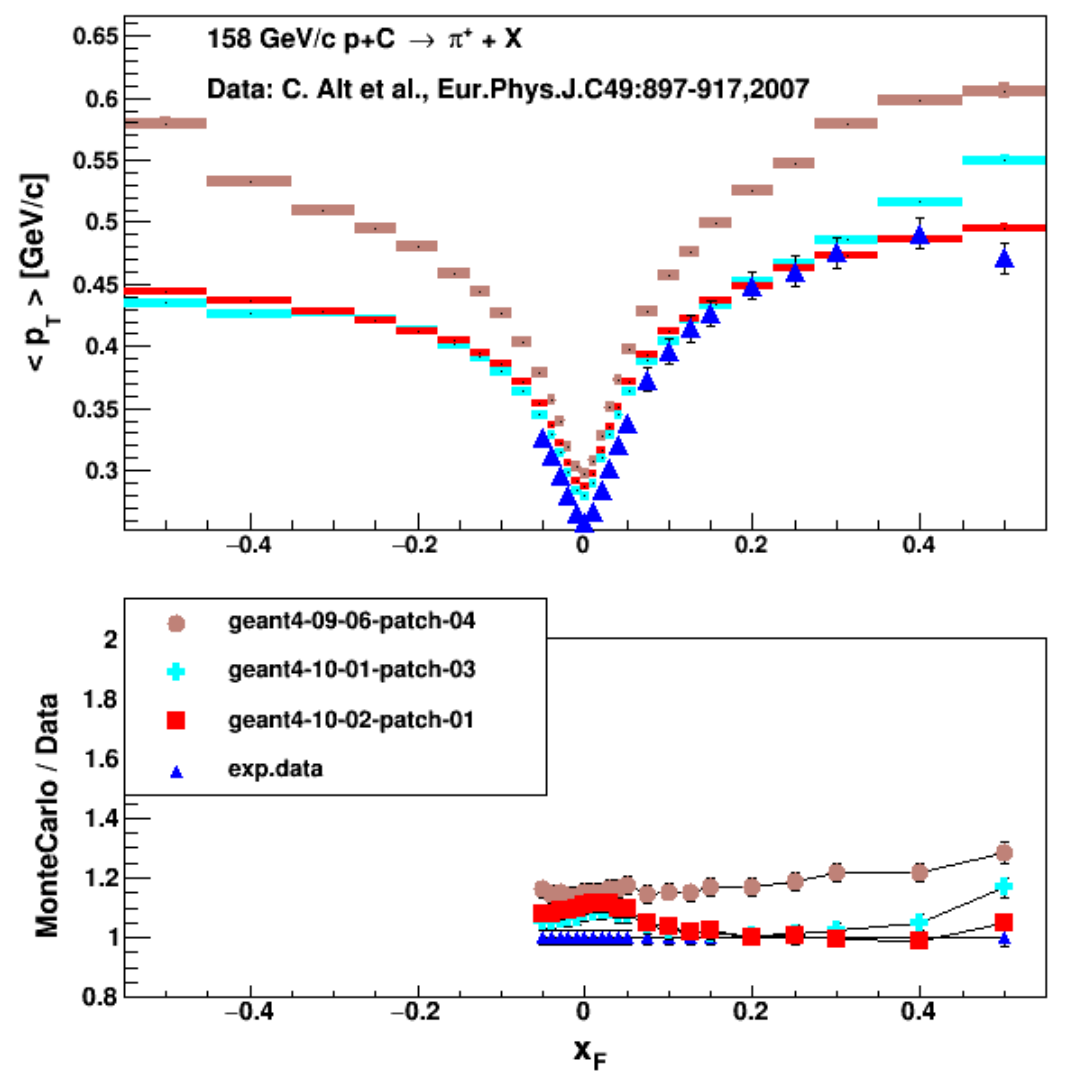

Figure 3: Comparison between NA49 20] results and Geant4 predictions for successive Geant4 versions for which the FTF and Bertini models have been improved. The $\pi^{+}$average momentum in the plane transverse to the particle beam, $p_{T}$, is presented as a function of Feynman $x$ $\left(x_{F}\right)$, for events with a final state $\pi^{+}$originating from a $158 \mathrm{GeV} / \mathrm{c}$ proton beam that hits a carbon target $\left(\mathrm{p}+\mathrm{C} \rightarrow \pi^{+}+\mathrm{X}\right) . x_{F}$ is defined as the ratio between the measured longitudinal momentum of the pion and the maximum value allowed by the kinematics of the collision, $x_{F}=p_{z}^{\pi} / p_{z \max }^{\pi}$. 

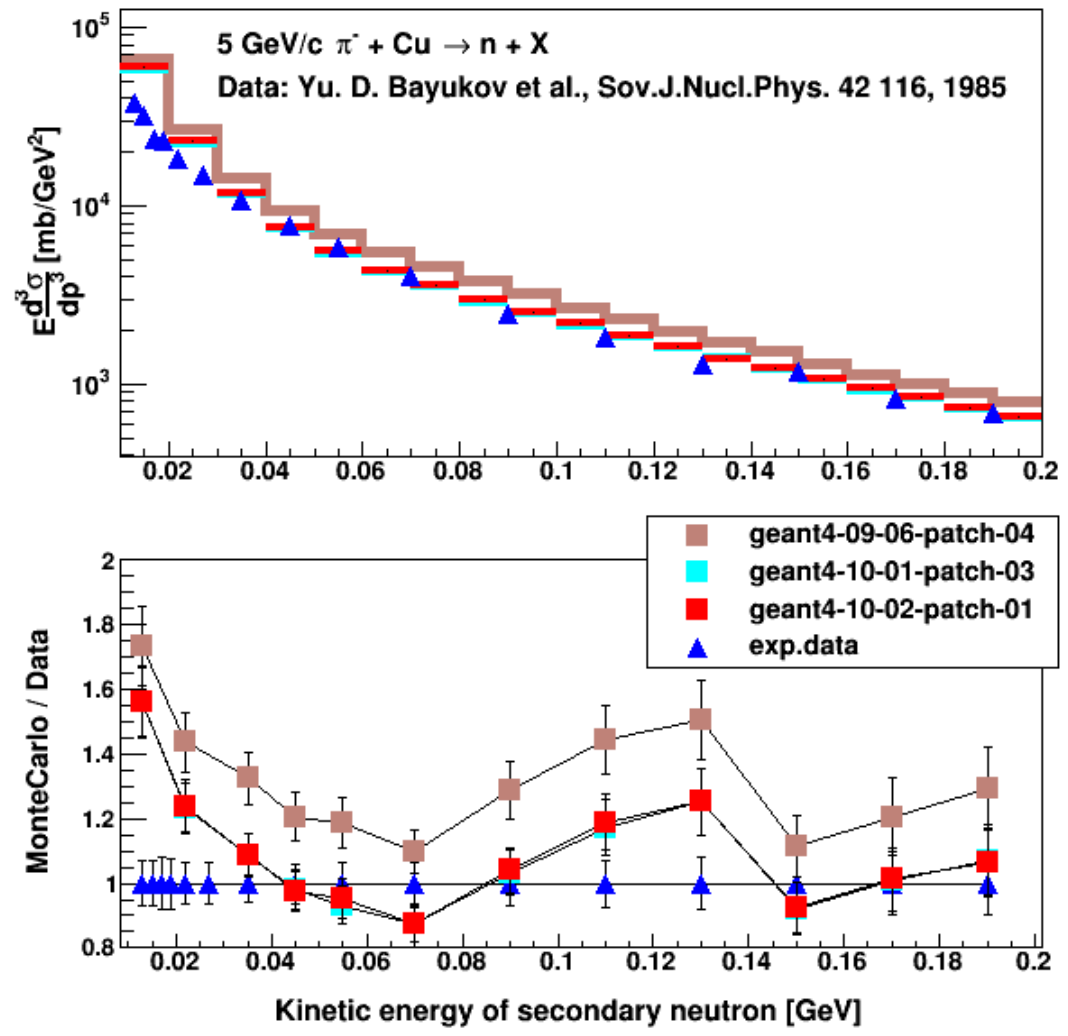

Figure 4: Comparisons between ITEP-771 27] experiment results and Geant4 predictions, for successive Geant4 versions for which the FTF and Bertini models have been improved. The $\pi^{-}(5 \mathrm{GeV})+\mathrm{Cu} \rightarrow \mathrm{n}+\mathrm{X}$ cross section is shown as a function of the neutron kinetic energy. 

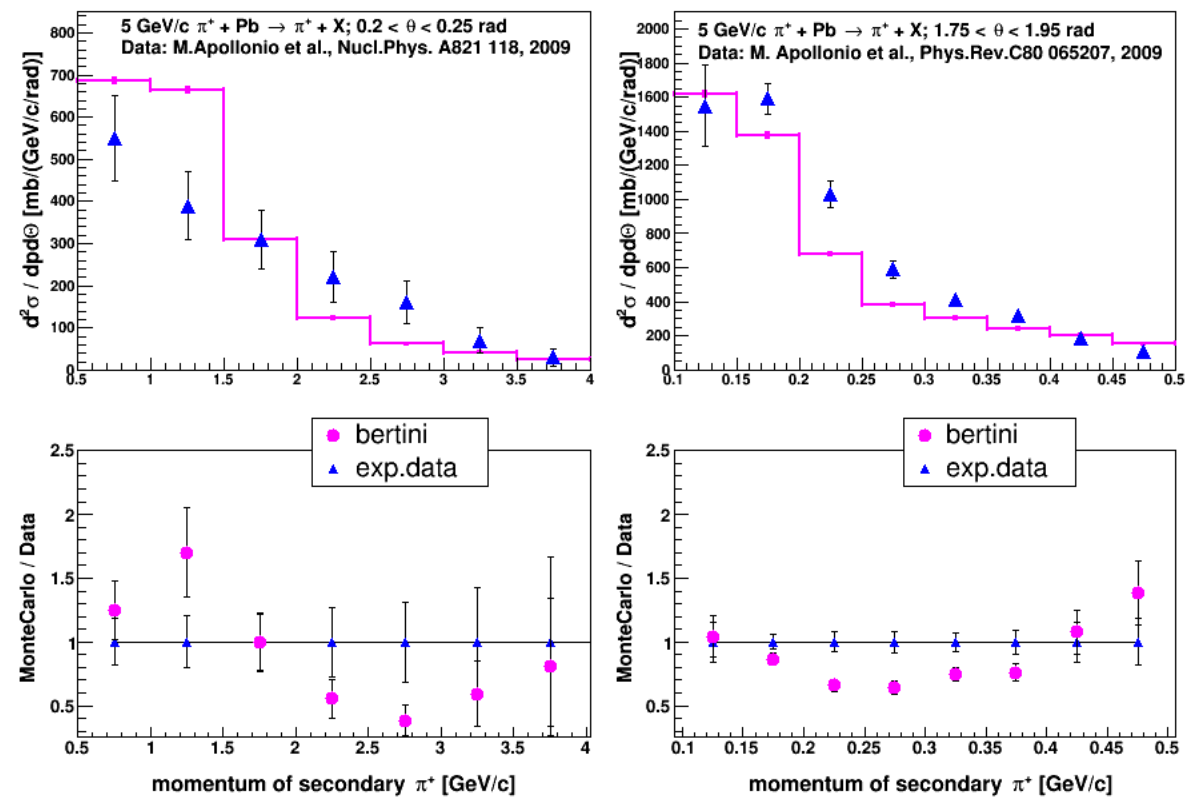

Figure 5: Polar angle distributions of the outgoing pion with respect to the direction of the incident pion beam versus the momentum of the secondary pion in $\pi^{+}(5 \mathrm{GeV})+\mathrm{Pb} \rightarrow \pi^{+}+\mathrm{X}$ collisions recorded by the HARP experiment 17 18. The comparison is made using Geant4 version 10.2.p01 with the Bertini model for two polar angle ranges. 
calorimeter (HCAL) in the region covered by a polar angle such that the pseudorapidity of the particles, $\eta \equiv-\tan (\theta / 2)<0.52$. The squares and circles correspond to different versions of Geant4, 10.0.p02 and 10.2.p02, and collections of physics models or physics lists. The FTFP_BERT_EMM list is the CMS experiment default Geant4 physics list (as of May 2017), based on the Bertini and FTFP models. As illustrated, the simulation models the track data within less than $5 \%$ in the $1-20 \mathrm{GeV} / \mathrm{c} p_{\text {Track }}$ range. Fig. 7 shows a similar measurement performed by the ATLAS experiment. ATLAS measured the $E / p$ from min-bias data, with $E$ the energy deposited by an isolated charged track in the calorimeter and $p$ the momentum measured in the tracker. The background subtracted mean ratio, $<E / p>_{\mathrm{COR}}$, is plotted as a function of the track momentum $p$ in two pseudorapidity regions, $|\eta|<0.6$ and $1.8<|\eta|<1.9$, using the 2010 and 2012 data samples. The measurements are compared to Geant4-based simulation predictions in the $p=0.5-30 \mathrm{GeV}$ range, using the FTFP_BERT and QGSP_BERT physics lists. In the kinematic region with small enough statistical uncertainties, the study proved that the simulation models the data to within $5 \%$ [29]. The previous examples are an illustration of how the increasing speed of computers during the last couple of decades allowed the LHC experiments to generate large-enough samples of simulated events to test different sets of Geant4 competing physics models and select those that describe the data best.

Another set of experimental results utilized by modern collider experiments to discriminate between different Geant 4 physics lists, and offer the Geant 4 collaboration guidance on how to assemble them from individual physics models, is the set of test beam single particle energy response and resolution measurements performed for different particles such as electrons, protons, and pions. Fig. 8 depicts comparisons of MC and data measurements of the response distribution, or response function, for $4 \mathrm{GeV}$ pions and $3 \mathrm{GeV}$ protons incident onto a solid angle slice of the CMS ECAL and HCAL calorimeters 28. Fig. 9 shows the mean pion and proton response as a function of the beam momentum, $p_{\text {beam }}$, for 2006 test beam data and the same two Geant 4 software versions and physics lists as in Fig. 8, The agreement is excellent, within uncertainties, over the whole range of particle momenta. For pions below $\sim 5 \mathrm{GeV}$, there seems to be a trend with the prediction overestimating the data by a few percent, although data and MC agree within uncertainties above $p_{\text {beam }}>3 \mathrm{GeV}$. For positive pions with beam energies of 20, 50, 100, and $180 \mathrm{GeV}$, Fig. 10 depicts the ATLAS calorimeter energy response, $E_{\text {total }} / E_{\text {beam }}$, and percentage resolution as a function of the beam energy $E_{\text {beam }}$. These 2000-2003 test beam results are compared to predictions from a Geant 4 version 10.1 simulation using different physics list options [30. The error bars are statistical only. The MC-to-data ratios show agreements within less than $2 \%$ for energy response and $10-15 \%$ for energy resolution.

The situation was radically different for the Tevatron experiments in the early nineties when computers were slower, simulation included poor approximations in exchange for time performance, and less advanced remote communication technology among scientists made it significantly more challenging to establish an international work program to understand and optimize the physics 
of GEANT3. Furthermore, the Tevatron test beam programs were very limited in scope and data-taking capabilities, in detriment of the $\mathrm{MC}$ validation exercise. Fig. 11 shows the electron-to-pion energy response ratio, $e / \pi$, versus beam energy as measured in the D0 Liquid Argon-Uranium calorimeter test beam experiment that took place in 1991 [31. In the case of CMS, statistical uncertainties in simulated data are negligible and the agreement is much better than for D0 in the energy range covered by the experiments. Furthermore, the CMS comparison extends to energies as low as $1 \mathrm{GeV}$ while the D0 measurement and comparison stops at $10 \mathrm{GeV}$. In CMS, the modeling of the momentum dependence of the single particle response is excellent, while in D0 $e / \pi$ flattens out much faster in MC than in data.

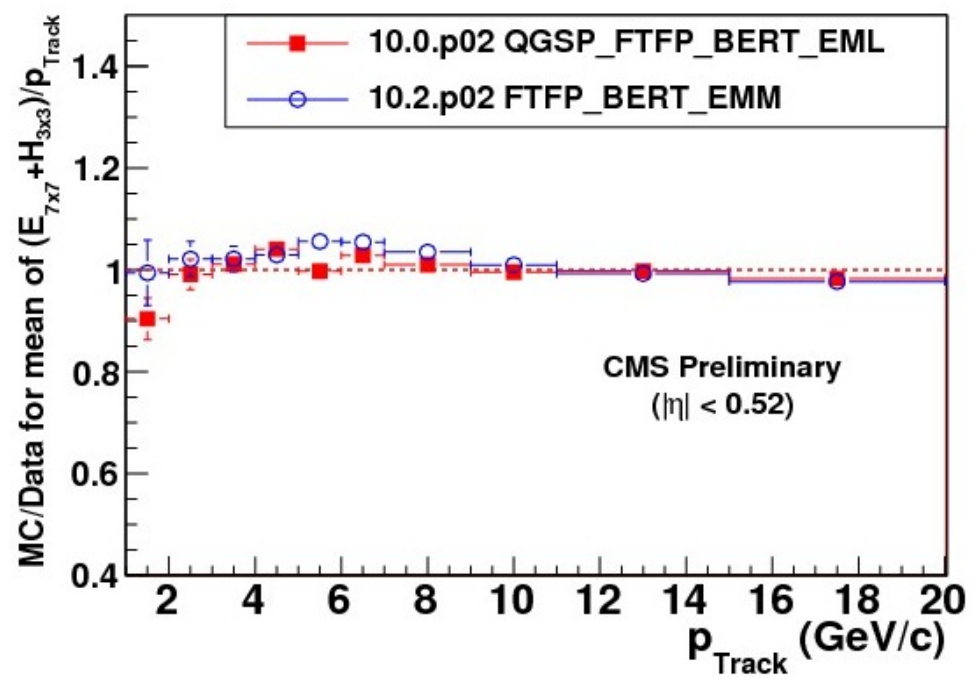

Figure 6: MC-to-data comparison results for isolated charged tracks in CMS min-bias events [28. The vertical axis displays the MC-over-data ratio of the ratio of the energy measured in the calorimeters over the momentum measured in the tracker, for a single isolated track. This ratio is measured as a function of the track momentum, $p_{\text {Track }}$. The simulation is performed for two different choices of Geant4 physics lists. Error bars are statistical uncertainties only. 

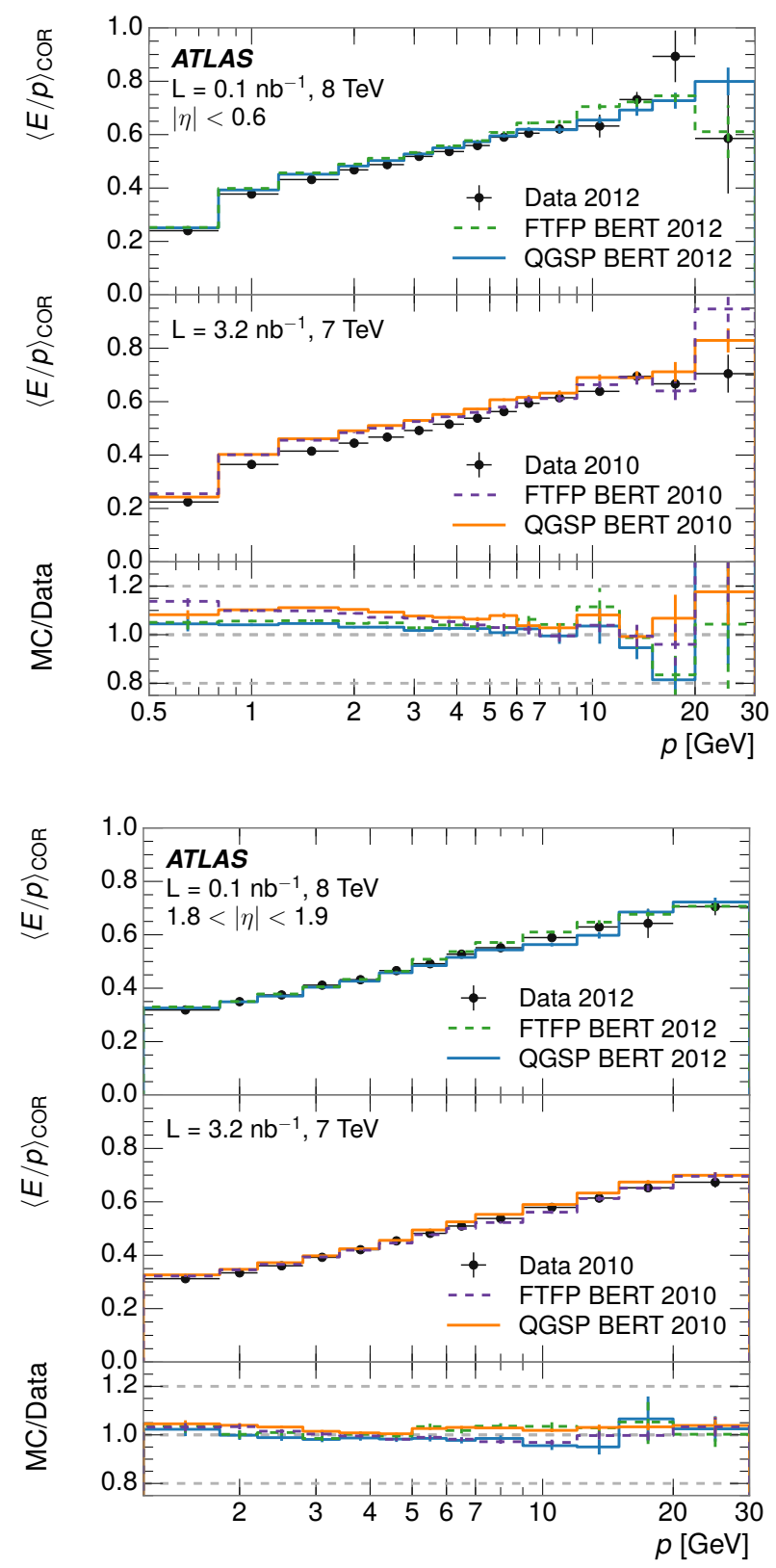

Figure 7: ATLAS measurement of $E / p$ from min-bias data, with $E$ the energy deposited by an isolated charged track in the calorimeter, and $p$ the momentum measured in the tracker. The background subtracted mean ratio, $\left\langle E / p>_{\mathrm{COR}}\right.$, is plotted as a function of the track momentum $p$ in two pseudorapidity regions, $|\eta|<0.6$ and $1.8<|\eta|<1.9$, using the 2010 and 2012 data samples. The measurements are compared to Geant4-based simulation predictions in the $p=0.5-30 \mathrm{GeV}$ range, using the FTFP_BERT and QGSP_BERT physics lists [29]. 

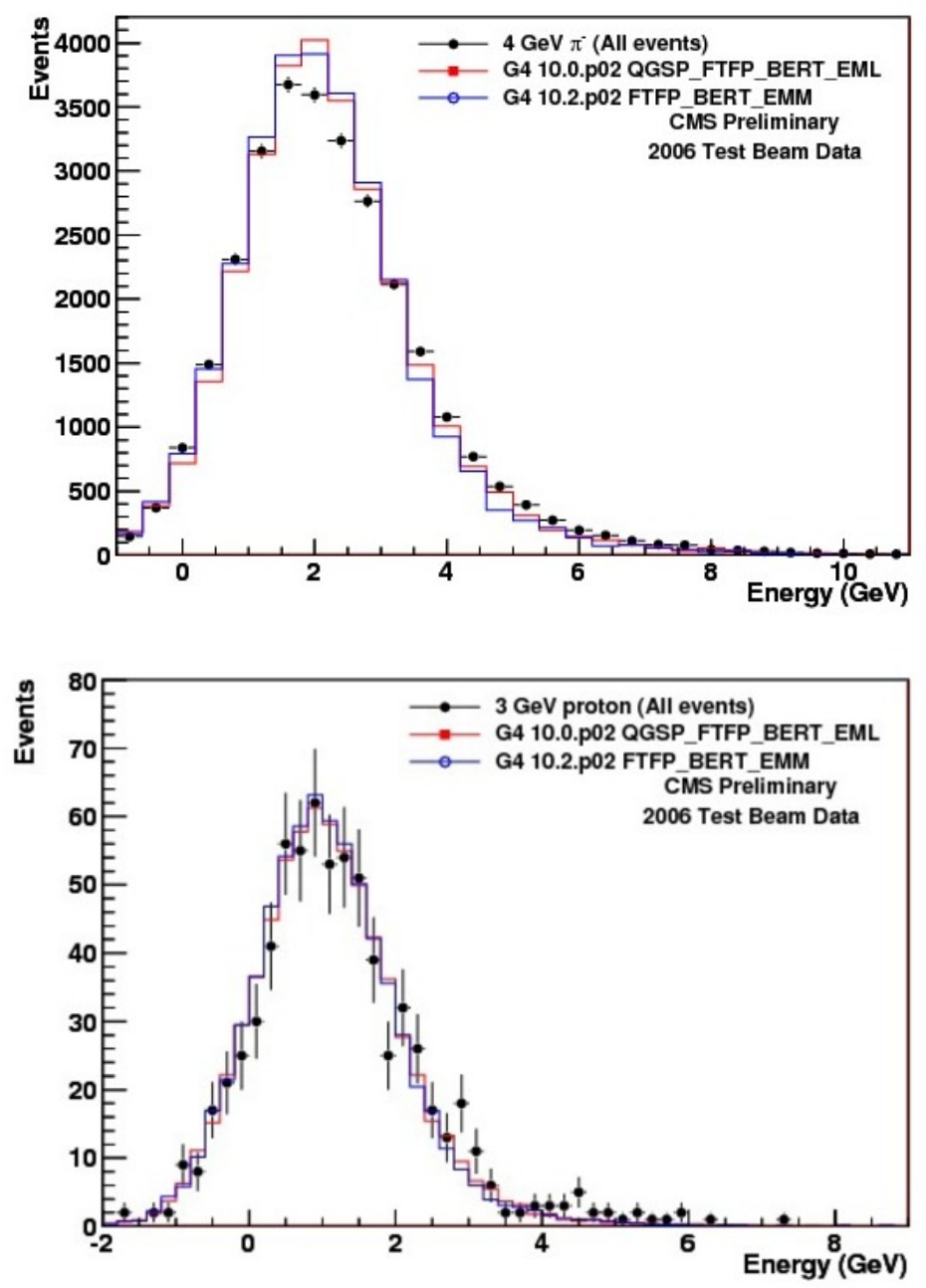

Figure 8: Comparisons of Geant4 and 2006 CMS test beam data measurements of the response distribution, or response function, for $4 \mathrm{GeV}$ pions and $3 \mathrm{GeV}$ protons incident onto a solid angle slice of the CMS ECAL and ECAL calorimeters 28. The simulation is performed for two different choices of Geant4 physics lists. 

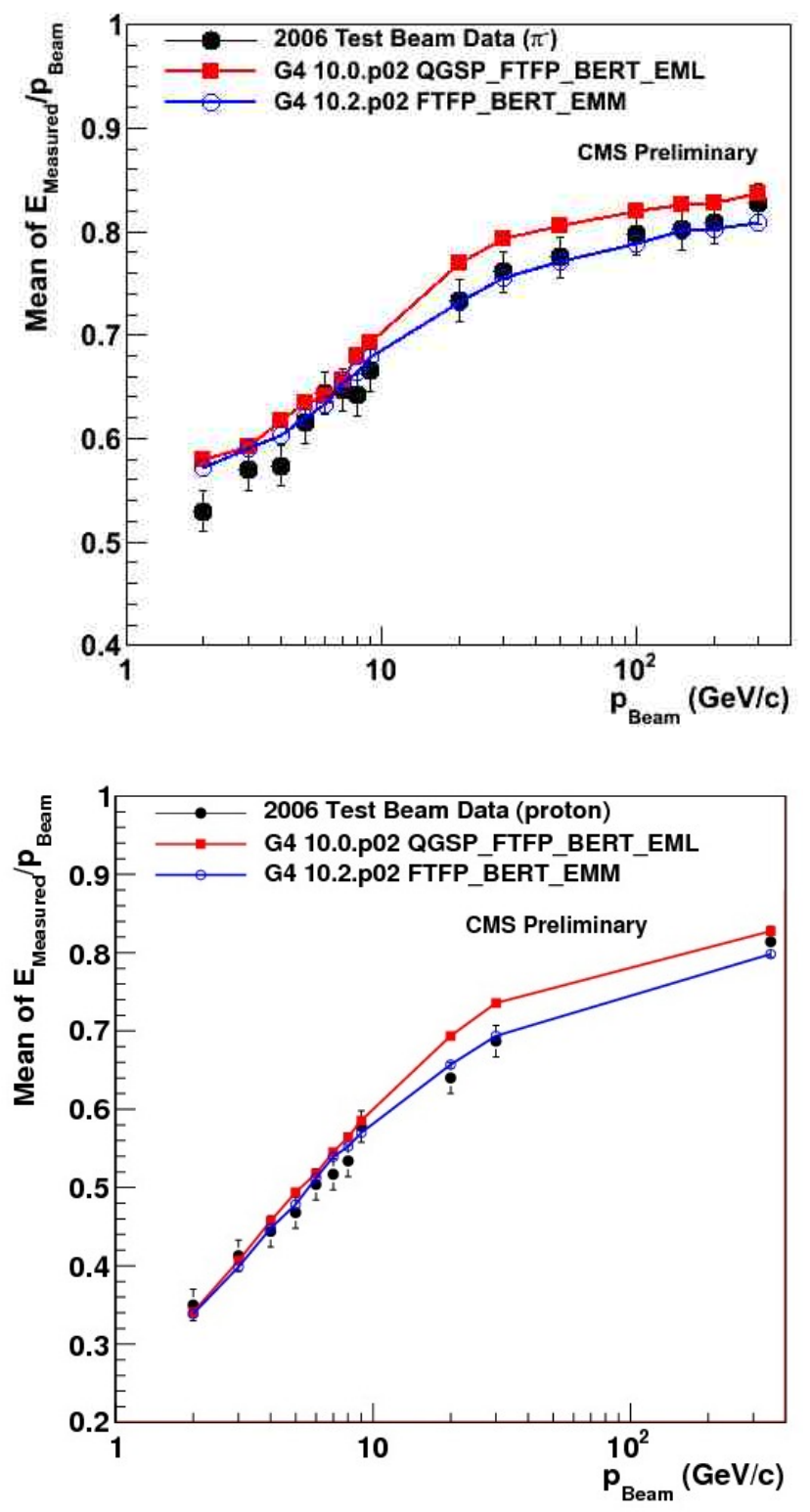

Figure 9: Mean pion and proton response as a function of the beam momentum, $p_{\text {beam }}$, as measured in the 2006 CMS test beam experiment 28. The simulation is performed for two different choices of physics lists. The error bars are statistical uncertainties only. 

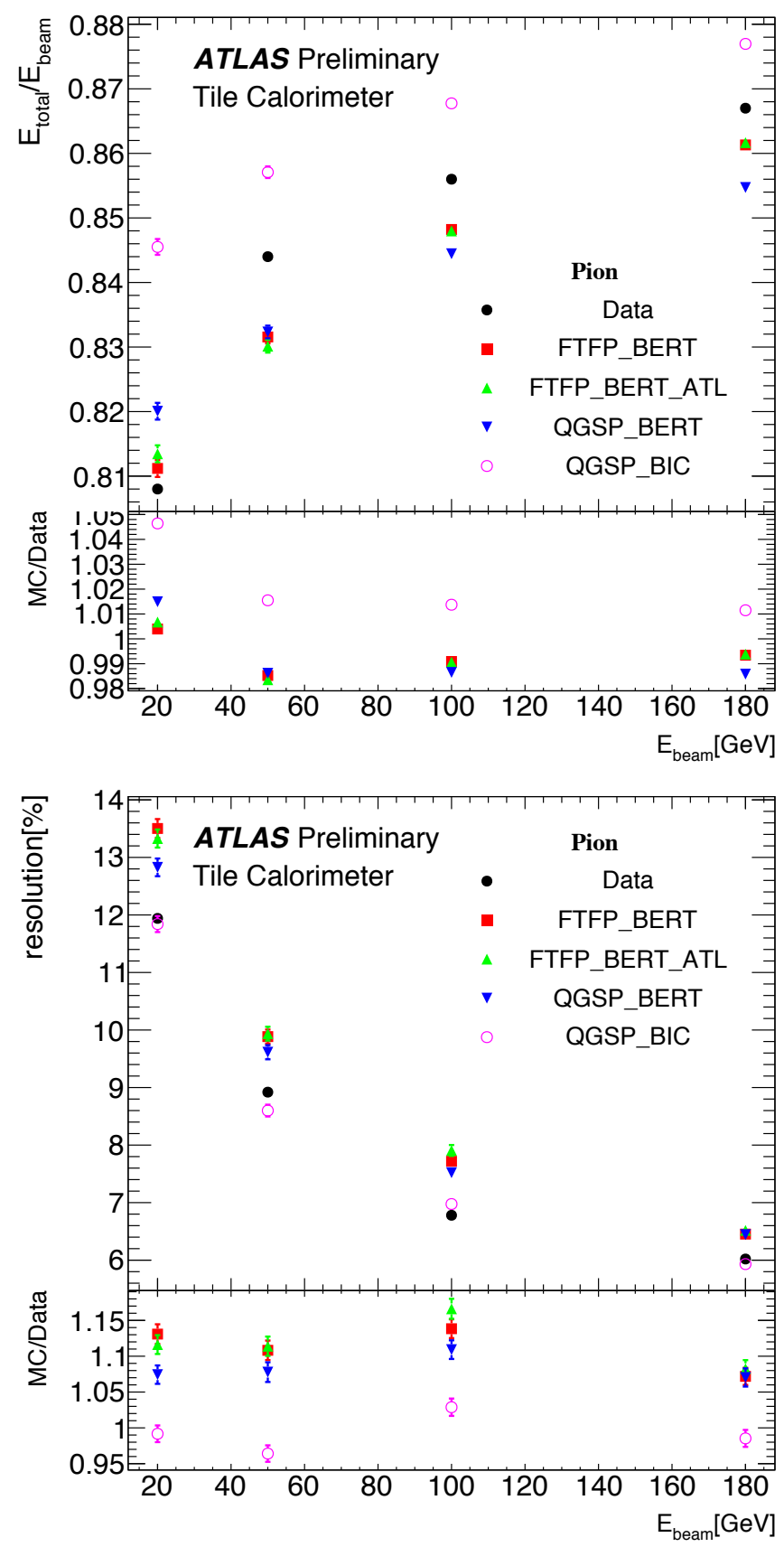

Figure 10: ATLAS calorimeter energy response, $E_{\text {total }} / E_{\text {beam }}$, and percentage resolution as a function of the beam energy $E_{\text {beam }}$. These 2000-2003 test beam results are compared to predictions from a Geant 4 version 10.1 simulation using different physics list options [30. The error bars are statistical only. 


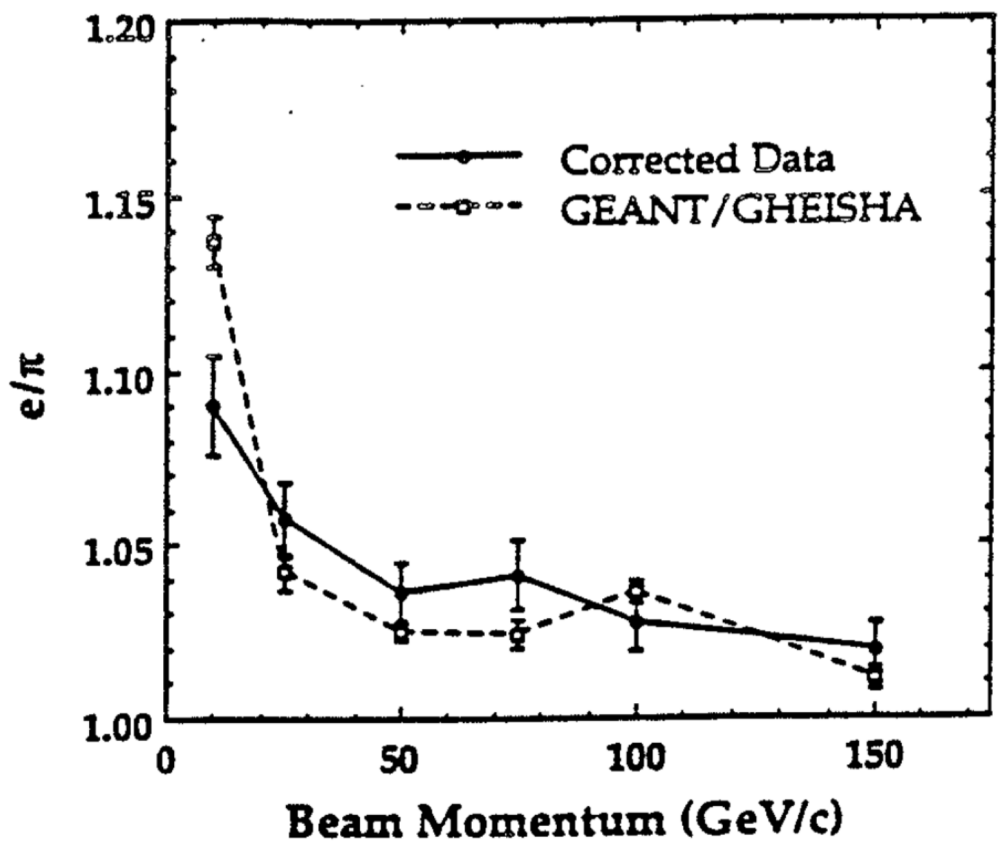

Figure 11: $e / \pi$ energy response ratio versus beam energy as measured in the D0 Liquid Argon-Uranium calorimeter test beam experiment that took place in 1991 [31. 


\section{Applications of Simulation to HEP Collider Experiments}

There are many applications of simulation to HEP collider experiments. One area is the analysis of the experimental data collected by the detectors and the interpretation of the resulting physics measurements in the light of theoretical predictions. Another use of simulation is in studies to design and optimize detectors for best physics performance. Simulation is also a critical tool utilized to develop calibration methods and reconstruction algorithms, as well as to preform stress-testing of the computing infrastructure.

\subsection{Simulation in Data Analysis}

Until recently, pure Geant-based simulation applications were rarely used to make a direct "MC truth" extraction of calibration factors, particle identification and reconstruction efficiencies, or backgrounds for particle searches. Experiments used well-tuned ParSim options instead, such as QFL or the GEANT3GFLASH tools developed in CDF. Pure Geant MC samples were either not accurate enough due to approximations to gain speed, they were statistically limited, or both. The situation changed significantly in the last few years when the availability of large samples of significantly more realistic simulated events became the norm in HEP experiments. As a result, MC-driven methods are being used with increasing frequency and confidence, as long as they are based on simulation code that has been thoroughly validated within systematic uncertainties, using thin target, test beam, and in situ experimental data. Data-driven methods, based on physics laws applied to real data, are still at the core of the derivation of calibration and correction factors applied to data measurements, while closure tests are essential to test the validity and precision of the methods. Closure tests are based on the comparison between the detector-level MC data, treated as if it were real data, and the Monte Carlo truth information associated with the particles of an event before they interacted with the detector.

\subsubsection{Data-driven Methods}

Data-driven methods are analysis techniques that use real experimental data, detector properties, and physics laws to perform detector calibration and alignment, estimate backgrounds in particle searches and, in general, determine correction factors applied to physics measurements. Simulation plays an essential role in the process of developing the methods, in the demonstration of their prediction power and the mitigation of biases, and in the derivation of the associated systematic uncertainties. A few examples of these data-driven techniques are described in the following paragraphs.

Object Balance for Jet Energy Calibration. Quark- and gluon-initiated jets are the most common physics objects in hadron collider experiments. The observed energy of jets in HEP detectors needs to be calibrated with a scale factor, which depends on the jet type and kinematics, and includes corrections for electronic noise, additional hard interactions in the same beam-beam crossing, detector response, and reconstruction algorithm effects. In a collider experiment, the 
response correction may be derived using conservation of momentum in the transverse plane, and the fact that the energy response and resolution are much better for electromagnetically interacting physics objects, such as photons or electrons, than they are for jets. The relatively small energy calibration factors for photons are similar to those for electrons, which are typically derived from $Z \rightarrow e^{+} e^{-}$samples. Once the photon scale is adjusted, jets may be calibrated using $p_{T}$ balancing, that is transverse momentum conservation in each event. To increase sample statistics and the accuracy of the measurement, jets in forward $\eta$ regions may be calibrated from di-jet events with one jet in the central region $(\eta$ close to 0$)$. As a bonus, the jet energy or $p_{T}$ resolution may be derived from the width of the asymmetry distribution, $A=\left(p_{T_{1}}^{\text {jet }}-p_{T_{2}}^{\text {jet }}\right) /\left(p_{T_{1}}^{\text {jet }}+p_{T_{2}}^{\text {jet }}\right)$. This is the approach used by the D0 experiment [32, 33], while CDF used QFL and its successor, the ParSim approach based on GEANT3 and GFLASH 34. The CMS experiment uses a hybrid approach where the MC truth prediction of the jet energy scale is adjusted by small factors derived from the above-mentioned data-driven techniques [35, in order to take into account the differences of jet energy scale in data and simulated events. ATLAS uses a similar calibration scheme based on both MC-driven and data-driven techniques 36.

Fig. 12 (top) shows the CMS data-to-MC ratio of the jet energy response as a function of jet $p_{T}$, determined from two different data-driven methods: $p_{T}$ balancing (solid squares) and Missing $p_{T}$ Fraction (solid circles). The Missing $p_{T}$ Fraction Method (MPF) is a variation of $p_{T}$ balancing, that uses the projection of the event transverse momentum imbalance vector onto the direction of the photon to estimate the response of the hadronic recoil. The message contained in Fig. 12 is that the normalization factor between the jet energy response measured in data and modeled in the CMS Full Simulation is approximately $\mathrm{SF}_{\mathrm{R}}=0.985$ with an uncertainty of less than $2 \%$. Moreover, the ratio shows that the $p_{T}$ dependence is flat to within the small uncertainties represented by the error bands. Although this ratio also depends on the jet flavor and pseudorapidity, the data-to-MC normalization or "scale" factors are in all cases small enough to allow CMS to follow the approach of extracting the jet energy response directly from $\mathrm{MC}$ truth information. In other words, the jet energy response applied as a correction to the jet energy in real collider data, $R_{\text {jet }}$, is calculated as $\mathrm{SF}_{\mathrm{R}} \times\left(p_{T}^{\text {reco }} / p_{T}^{\text {part }}\right)$, where $p_{T}^{\text {reco }}$ is the detector level jet $p_{T}$ obtained from the reconstruction software algorithms applied to $\mathrm{MC}$ events, and $p_{T}^{\text {part }}$ is the jet true $p_{T}$, with "true $p_{T}$ " referring to the $p_{T}$ of the particle-level jet, after fragmentation and hadronization, before it hit the detector. The $\mathrm{SF}_{\mathrm{R}}$ factor puts MC and data in the same footing, by shifting $R_{\text {jet }}$ in MC to model what was measured in data. This approach is significantly more accurate because, since the same data-driven method is applied to both MC and data, the uncertainty in the ratio is much smaller than that in the numerator and denominator, given that most uncertainty components are correlated and cancel. It is not the jet response what is measured but how different the measurements are in data and MC.

Fig. 12 (bottom) shows the asymmetry distribution $A$ for a CMS sample 
of di-jet events from where the jet $p_{T}$ resolution is measured. The agreement between MC and data is excellent except in the non-Gaussian tails of the distribution, which are very difficult to model in MC because they come from non-linear contributions to the detector response. Although the CMS calorimenter system (ECAL+HCAL) is undercompensating, with an $e / h>2(e, h$ are the response to the energy deposited by an incident hadron through electromagnetic and nuclear interactions respectively), non-linear behavior is corrected to a large extent during calibration and through the use of tracking information by the particle flow algorithm [37. As in the case of the jet energy response, MC truth resolutions, properly adjusted with data-to-MC scale factors, are utilized in data analysis.

As in CMS, one of the methods utilized by ATLAS to perform the jet calibration is MPF. Fig. 13 (top) shows the measured MPF response in a photon plus jets sample, as a function of $p_{T}^{\gamma}$ in the central pseudorapidity region [38. Also for ATLAS, the MC models the MPF response very accurately over the whole kinematic range of interest, deviating slightly from a 0.98 flat data-to$\mathrm{MC}$ ratio in the lowest and highest extremes of the range. The shaded band in Fig. 13 (bottom) shows the total uncertainty in the data-to-MC MPF response ratio, which is less than $1 \%$ for $p_{T}^{\gamma}>70 \mathrm{GeV}$. The excellent data-to-MC agreement for the asymmetry distribution, $A$, is illustrated in Fig. 14 (top) for the ATLAS experiment [39]. Although calorimeter jets are used in this example, the ATLAS asymmetry distribution is reasonably well described by a Gaussian function, since the $e / h=1.37$ value for the ATLAS calorimeter system does not deviate that much from perfect compensation, $e / h=1$. Still, the modeling of the residual non-linear behavior contributing to the tails is difficult and both the data measurement and the MC prediction deviates from a perfect Gaussian distribution in the tails. Fig. 14 (bottom) shows the measured jet energy resolution, $\sigma\left(p_{T}\right) / p_{T}$, versus the average jet $p_{T}$ of the two jets in the di-jet sample used for the derivation. The agreement between $\mathrm{MC}$ and data for the di-jet balance method is impressive, while the agreement when using the bisector method is within $10 \%$. The bisector method [39] is a variant of the di-jet balance technique, and measures the variance of the $p_{T}$ balance vector projected along an orthogonal coordinate system in the transverse plane, where one of the axes is chosen in the direction that bisects the azimuthal angle formed by the two leading jets.

Control Samples for Background Estimation. One essential aspect of a search for a new particle, or a characterization of a known particle, is the selection of a signal-enhanced sample, where the particle under study represents the signal, and all other physics processes resulting in similar final states or detector signatures represent the backgrounds. Control Samples (CS's) or control regions (CR's) are background-only samples, or regions of phase space, used to estimate the background contributions in a signal region (SR) from a combination of measurements in the CR, event properties, and physics laws. For example, it may be known that a given functional form fits well a Standard Model process which is a background to a beyond-the-Standard-Model (BSM) signal under 

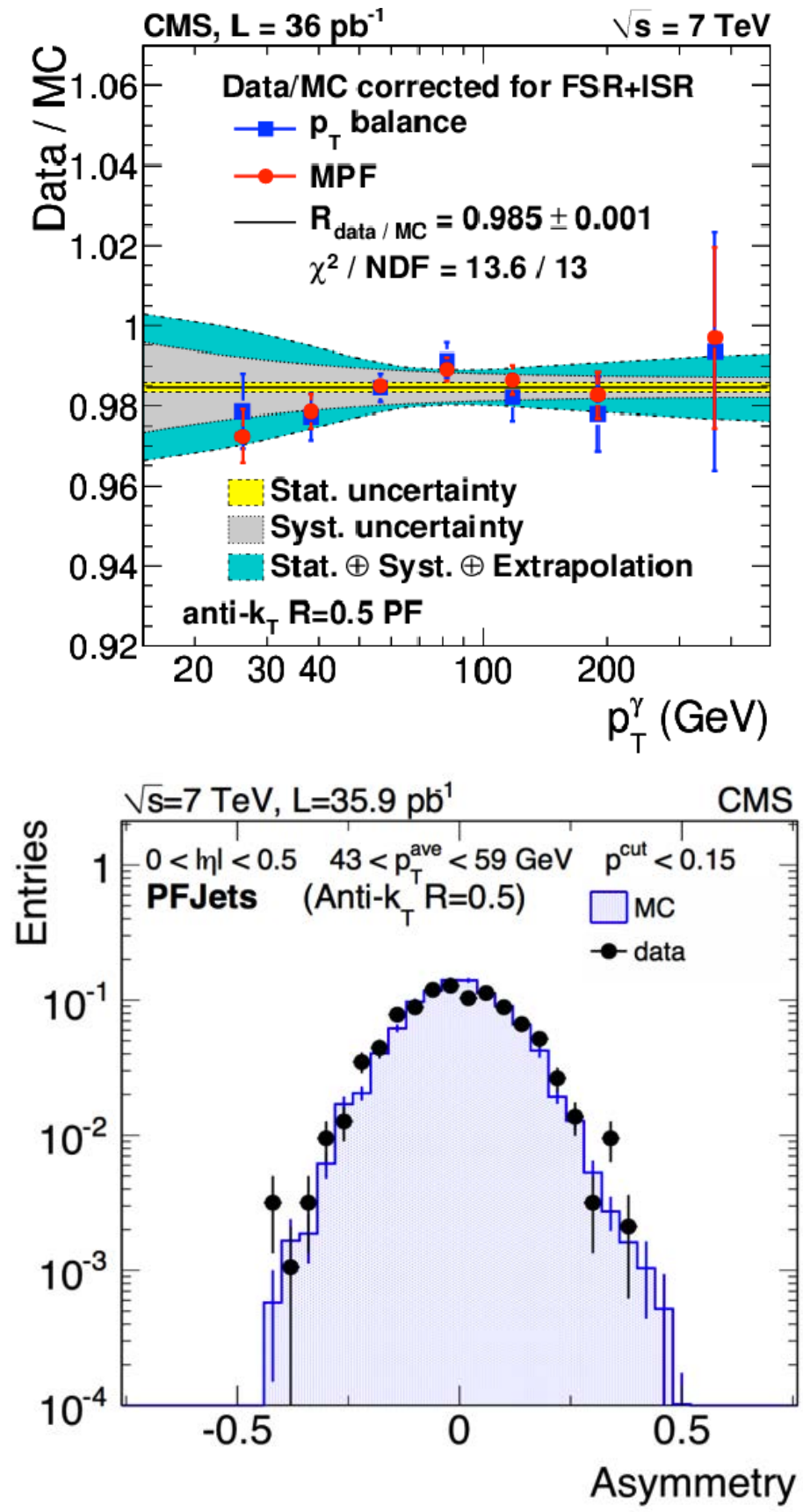

Figure 12: Top: CMS data-to-MC ratio of the jet energy response as a function of jet $p_{T}$, determined from two different data-driven methods [35]: $p_{T}$ balancing and Missing $p_{T}$ Fraction. Bottom: Asymmetry distribution, $A=\left(p_{T_{1}}^{\text {jet }}-p_{T_{2}}^{\text {jet }}\right) /\left(p_{T_{1}}^{\text {jet }}+p_{T_{2}}^{\text {jet }}\right)$, for a CMS sample of di-jet events from where the jet $p_{T}$ resolution is measured. The asymmetry variable measurement shows statistical errors only. 

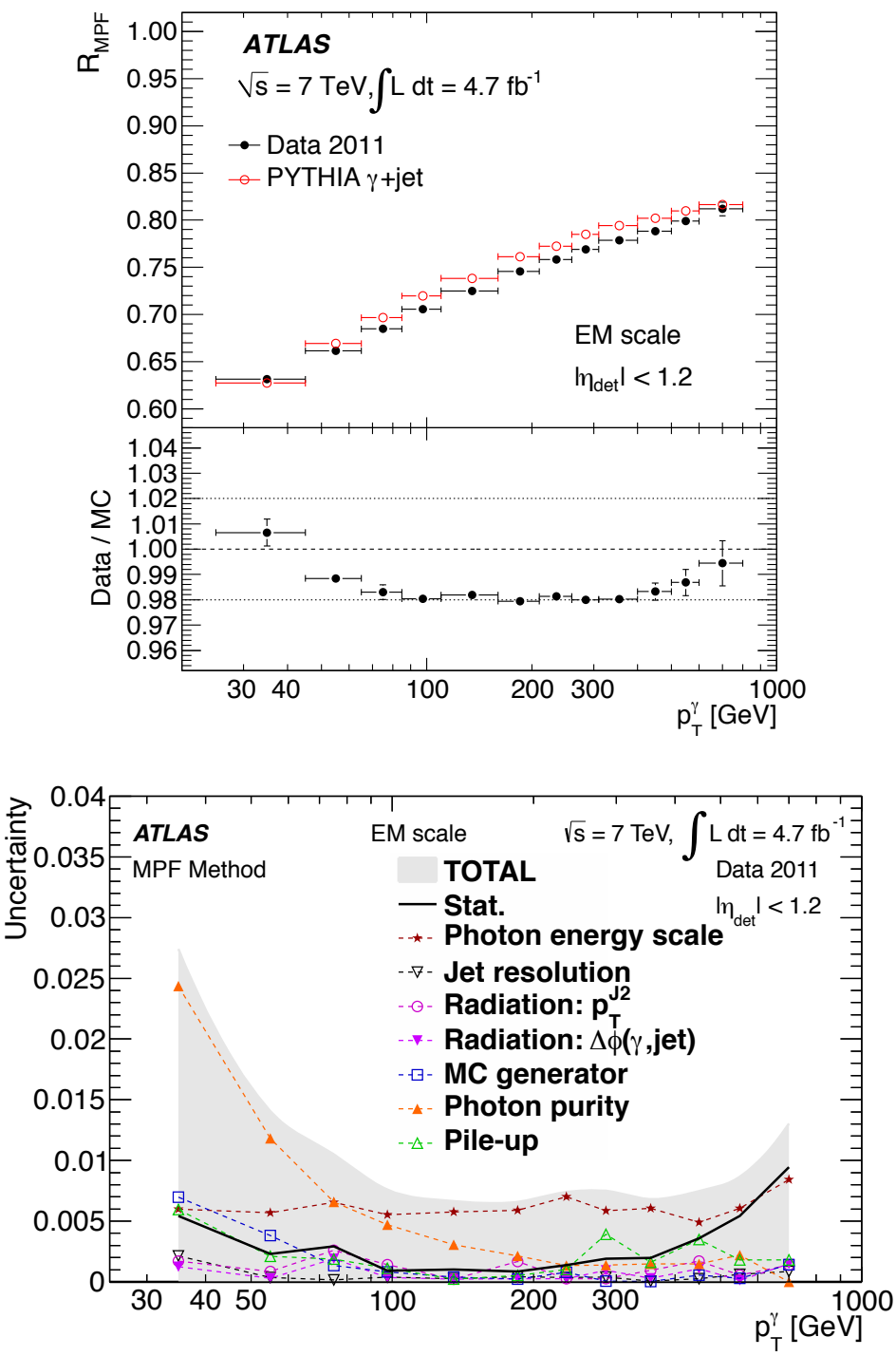

Figure 13: Top: Jet response measured in ATLAS with the MPF method applied to photon plus jets events. Photons are calibrated to account for the EM scale, derived from a $Z \rightarrow e^{+} e^{-}$ data sample. The data-to-MC response ratio is shown in the bottom inset of the figure. Only the statistical uncertainties are shown. Bottom: Systematic uncertainties on the MPF response data-to-MC ratio, with photons calibrated to the electromagnetic (EM) scale. See Ref. [38. 

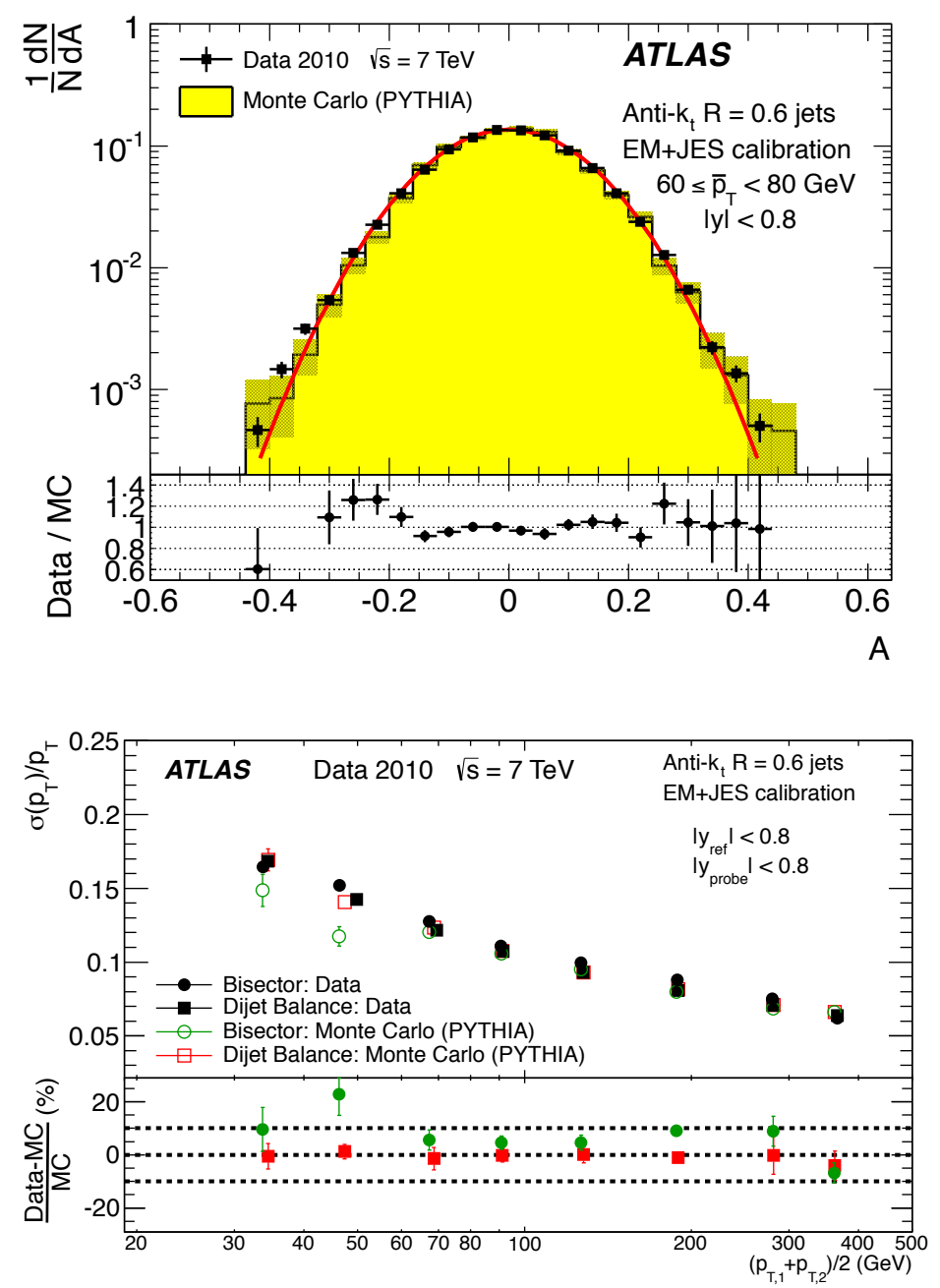

Figure 14: Top: Asymmetry distribution, $A=\left(p_{T_{1}}^{\text {jet }}-p_{T_{2}}^{\text {jet }}\right) /\left(p_{T_{1}}^{\text {jet }}+p_{T_{2}}^{\text {jet }}\right)$, for an ATLAS sample of di-jet events. Bottom: ATLAS jet $p_{T}$ resolutions measured using the di-jet balance and bisector methods. In both plots, error bars are statistical and the lower panels show data-toMC ratios to illustrate the level of agreement between simulation and data. See Ref. 39. 
study. To estimate the background in the SR, the function may be fit to the data in the CR and then extrapolated to the SR to make the prediction.

Fig. 15 illustrates how MC samples are used to establish the boundaries of CR's and SR's for use in the data-driven prediction of the QCD background to multi-jet final states in a CMS Supersymmetry (SUSY) [0] search 41]. SUSY is a theory based on a symmetry that relates bosons and fermions, offering a solution to the Higgs boson hierarchy problem, and predicting the unification of gauge couplings and a dark matter candidate. Fig. 15 shows the minimum azimuthal angular distance $\min \Delta \phi\left(\right.$ jet $\left._{1,2,3}, H_{T}^{\text {miss }}\right)$ between the three leading jets in the event and the event $\vec{H}_{T}^{\text {miss }}$, defined as the the negative vector sum of the $p_{T}$ 's of all jets in the event [42. This angle is plotted as a function of $H_{T}^{\text {miss }}$, the absolute value of the vector. For QCD background events, $\min \Delta \phi\left(\right.$ jet $\left._{1,2,3}, H_{T}^{\text {miss }}\right)$ is small and $H_{T}^{\text {miss }}$ low, since the $p_{T}$ imbalance comes from detector response and resolution effects, and tends to be aligned with the two approximately back-to-back leading jets. For certain SUSY models, the two leading jets and two weakly interacting BSM stable particles, called neutralinos $\left(\tilde{\chi}_{1}^{0}\right)$, tend to be in opposite hemispheres of the transverse plane, generating high missing $p_{T}$ in the event as well as a large $\min \Delta \phi\left(\right.$ jet $\left._{1,2,3}, H_{T}^{\text {miss }}\right)$. The data-driven technique illustrated in Fig. 15 with a $\mathrm{MC}$ sample is known as the factorization or ABCD method. It consists of identifying three CR's (A, B, and D) and a SR (C), which corresponds to events with a large angular distance and high $H_{T}^{\text {miss }}$. The background in $\mathrm{C}$ may be estimated as $N_{C}=\left(N_{B} / N_{A}\right) \times N_{D}$, if the variables are uncorrelated and as $N_{C}=f\left(H_{T}^{\text {miss }}\right) \times N_{D}$ if they are correlated, with $f\left(H_{T}^{\text {miss }}\right)$ determined from an extrapolation of a fit of an appropriate function to data in the CR.

Tag-and-Probe Method for Efficiency and Fake Rates. The principle of the tagand-probe method, when applied to measure particle reconstruction and identification efficiencies, is to use the a priori knowledge of the identity of a reconstructed physics object (Tagged Object), for example a known resonance, and ask the question on the fraction of the times a given Probe Object is identified by a reconstruction and identification algorithm correctly. The method is also used to measure trigger efficiencies, or the probability for a hardware or software trigger system to flag an event it is designed to identify from all collisions occurring in the experiment. Particle isolation efficiency is the probability for a particle to pass a requirement of isolation with respect to other particles in the event, and fake rate is the probability for a particle to be miss-identified as a different particle by a software algorithm. Both may also be measured from real data using the tag-and-probe method. For example, the probability that an electron is missidentified as a photon, the $e \rightarrow \gamma$ fake rate, may be derived from three di-object samples where each of the two objects has been identified either as an electron or a photon: $e^{+} e^{-}, e^{+/-} \gamma, \gamma \gamma$. The di-object invariant mass distribution will show a distinct peak centered at the mass value of the $Z$ boson. In average, once the non- $Z$ di-electron continuous and monotonically decreasing background is subtracted, all the events within the $m_{Z} \sim 91.2 \mathrm{GeV}$ peak are bound to be electrons, no matter whether they have been identified as such or as $e \gamma$, or $\gamma \gamma$, 

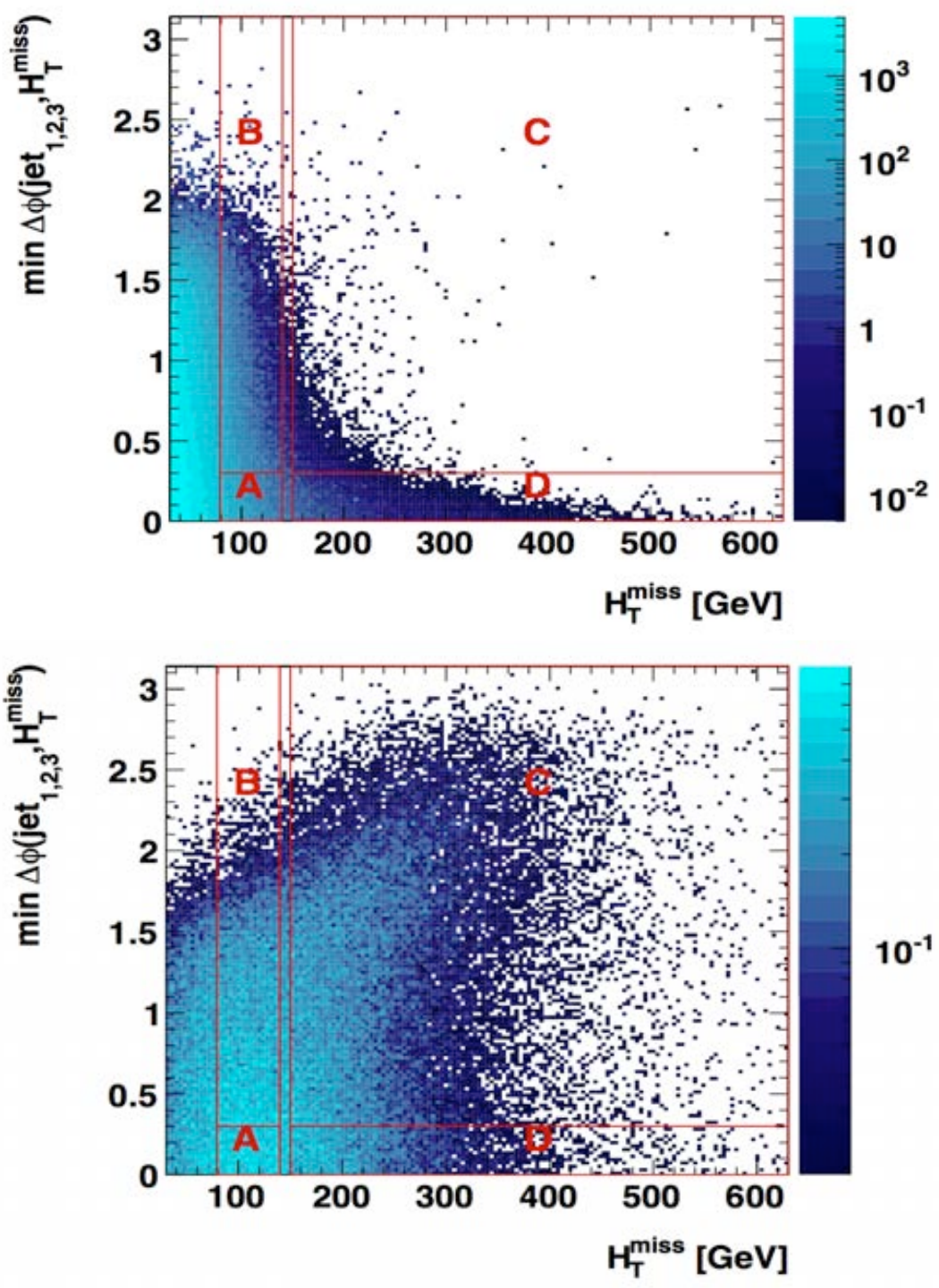

Figure 15: Illustration of the ABCD or factorization method for multi-jets (QCD) background estimation. The minimum azimuthal angular distance between the three leading jets in the event, min $\Delta \phi\left(\right.$ jet $\left._{1,2,3}, H_{T}^{\text {miss }}\right)$, is shown as a function of the event $H_{T}^{\text {miss }}$, for a QCD background only sample (top) and a SUSY signal sample (bottom). It is apparent that the A, B, and $\mathrm{D}$ regions are dominated by the background, while the $\mathrm{C}$ region is background depleted and signal enhanced 42 . 
because the decay rate of $Z$ bosons to photons is negligible. In the example, the electrons are the tagged objects and the photons the probe objects. The photons are fake photons, in reality electrons miss-identified as photons. Thus the $e \rightarrow \gamma$ fake rate is calculated as $f_{e \rightarrow \gamma}=\left(N_{e e}^{\gamma}+N_{e \gamma}^{\gamma}+N_{\gamma \gamma}^{\gamma}\right) / N_{T O T}^{e m}$, where $N_{e e}^{\gamma}$, $N_{e \gamma}^{\gamma}, N_{\gamma \gamma}^{\gamma}$ are the number of photons in the $e^{+} e^{-}, e^{+/-} \gamma$, and $\gamma \gamma$ samples and $N_{T O T}^{e m}$ is the total number of electromagnetic interacting objects, either $e$ or $\gamma$. Another application of tag-and-probe is the determination of the $\tau$-lepton reconstruction plus identification efficiency from a di-lepton sample. In this case, one lepton is identified as a muon and the other lepton as a hadronically-decaying $\tau\left(\tau_{\text {had }}\right)$ using the standard identification selections. When computing the dilepton invariant mass distribution, a $Z$ boson peak is visible, populated with the events where the $\mu$ comes from a leptonically-decaying $\tau\left(\tau_{\text {lep }}\right)$. Here, the $\tau_{\text {had }}$ is the tagged object and the muon the probe object. The $\tau$ reconstruction plus identification efficiency is calculated as $\varepsilon_{\tau}^{\text {reco+id }}=N_{\text {pass }}^{\text {evt }} /\left(N_{\text {pass }}^{\text {evt }}+N_{\text {fail }}^{\text {evt }}\right)$, where $N_{\text {pass }}^{\text {evt }}, N_{\text {fail }}^{\text {evt }}$ are the number of events passing and failing the requirement that there are two $\tau$ leptons. Following the same procedure described for the jet energy corrections, experiments typically extract object identification efficiencies directly from MC truth predictions and adjust them with scale factors computed as ratios between the data-driven efficiencies obtained from real data and MC samples, respectively.

Fig. 16 shows a data-to-MC comparison of the CMS muon reconstruction

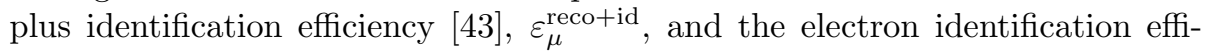
ciency for medium electrons [44, $\varepsilon_{e}^{\text {id }}$, measured with the tag-and-probe method. In this particular case, the method is referred to as tight-and-loose, because it utilizes $Z \rightarrow \mu^{+} \mu^{-}, J / \psi \rightarrow \mu^{+} \mu^{-}$, and $Z \rightarrow e^{+} e^{-}$samples to measure the efficiencies, where the tagged lepton is selected with a stringent (tight) criteria and the probed lepton with a relaxed (loose) criteria. Efficiencies are not defined the same way for all physics objects and in all experiments, and a good understanding of the definition details is important for their correct utilization in physics analysis. For example, the CMS muon efficiency shown in Fig. 16 refers to reconstruction plus identification (or muon sample selection) efficiency. It is the conditional probability of identifying a muon with a looser or tighter selection criteria given that a track in the tracker system exists. The CMS electron efficiency in Fig. 16 accounts for identification only, and must be multiplied by the reconstruction only efficiency to yield the combined reconstruction plus identification efficiency.

ATLAS also uses the tag-and-probe method to measure $\varepsilon_{\mu}^{\text {reco+id }}$ [45] and $\varepsilon_{e}^{\text {reco+id }}$ [46] in $Z \rightarrow \mu^{+} \mu^{-}, J / \psi \rightarrow \mu^{+} \mu^{-}$, and $Z \rightarrow e^{+} e^{-}$samples. The muon efficiency in Fig. 17 is the conditional probability of reconstructing a muon that successfully combines inner detector and muon system information (CB), given that a track is found in the inner detector. The ATLAS electron efficiency in Fig. 17 is the product of the reconstruction and identification efficiencies.

Except in the case of the CMS muon efficiency, all other plots in Figs. 16, 17 show an inset with the data-to-MC ratio and total uncertainty, which translates into the scale factors utilized to adjust the MC truth efficiencies used in physics 

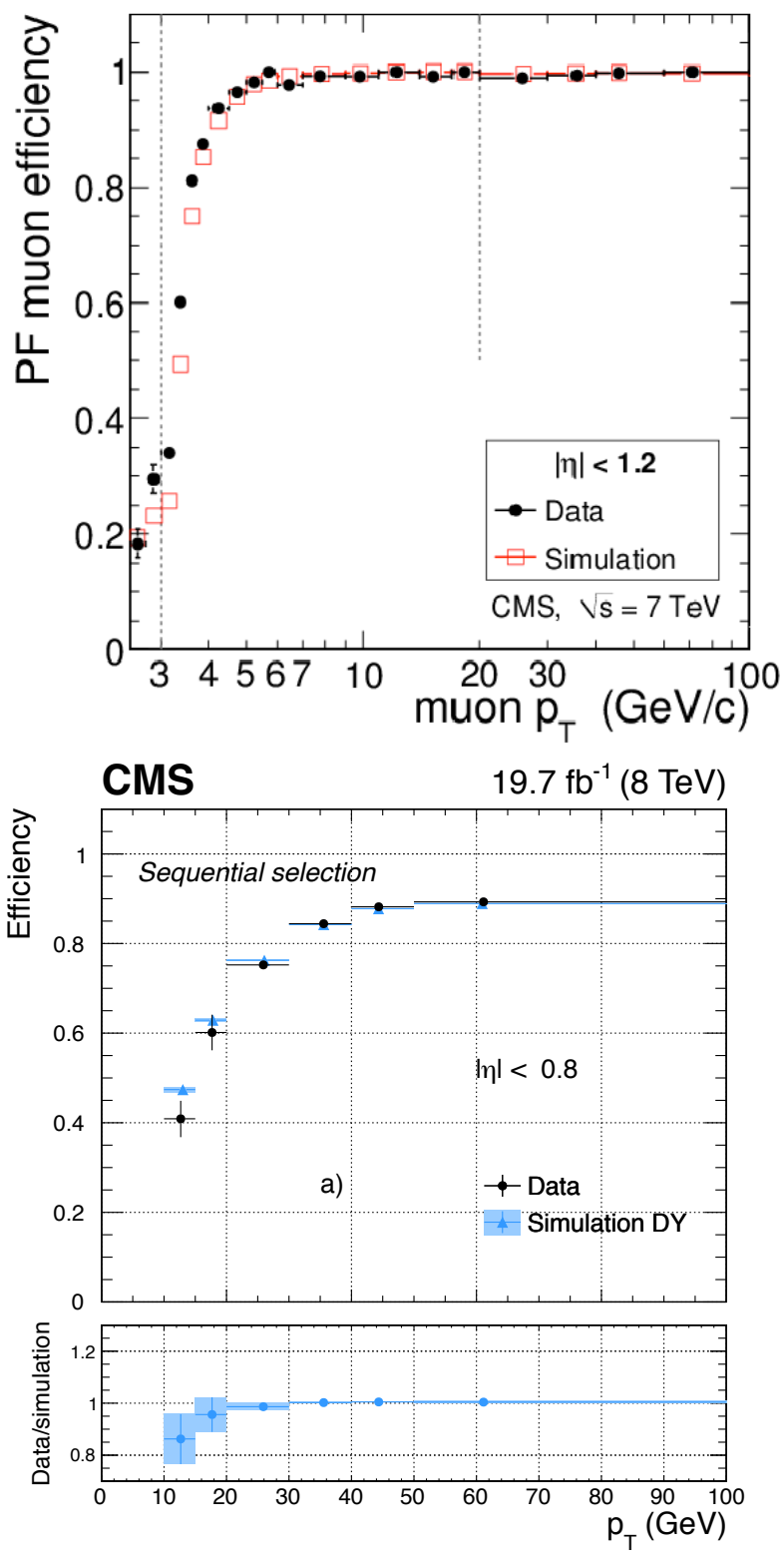

Figure 16: Data-to-MC comparison of the CMS muon reconstruction plus identification efficiency, $\varepsilon_{\mu}^{\text {reco+id }}$ [3] (top), and the electron identification efficiency for medium electrons, $\varepsilon_{e}^{\text {id }} 44$ (bottom), measured with the tag-and-probe method. The lower panel displays the data-to-MC scale factors used to correct the electron MC prediction for use in data analysis. The uncertainties shown in the muon efficiency plot are statistical only, and the uncertainty band in the electron efficiency plot represents statistical and systematic errors on the method added in quadrature. 

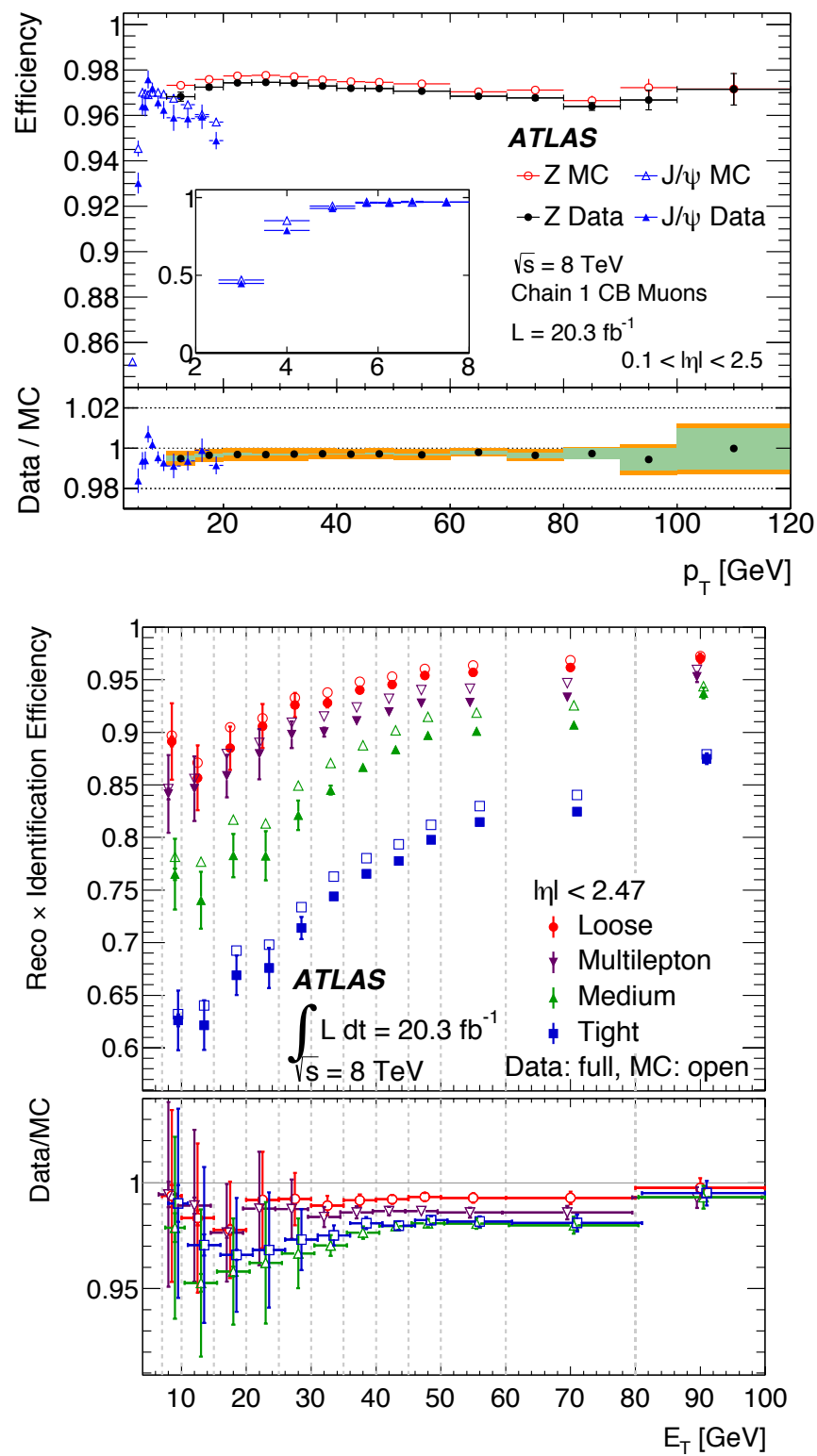

Figure 17: Data-to-MC comparison of the ATLAS muon [45] (top) and electron [46] (bottom) reconstruction plus identification efficiencies, $\varepsilon_{\mu / e}^{\text {reco+id }}$, measured with the tag-and-probe method. The lower panels show the data-to-MC scale factors used to correct the MC predictions for use in data analysis. The green uncertainty band depicts statistical uncertainties only, while the orange band also includes the systematic uncertainties. In the case of the electrons, the inner error bars are statistical and the outer bars depict the total error. 
analysis. For muons with $p_{T}>5 \mathrm{GeV}$, the agreement between simulation and data is excellent for both experiments, while for electrons ATLAS shows a small disagreement of the order of 1-2\% depending on the selection criteria, larger for $E_{T}<40 \mathrm{GeV}$, which is due to mismodeling of the shower shape in the forward calorimeter. In the case of CMS, once the electron reconstruction efficiency, which is not shown and varies in the $90-97 \%$ range for $15-100 \mathrm{GeV}$ electrons, is multiplied by the medium electron identification efficiency, the results are similar to ATLAS's. However, CMS's coverage is restricted to the central $\eta$ region in this particular plot, which shows excellent data-to-MC agreement above $30 \mathrm{GeV}$, and a trend towards $\mathrm{MC}$ overestimation en the $10-30 \mathrm{GeV}$ range.

\subsubsection{Closure Tests}

While the use of accurate simulation increases the chances that the software developed with it performs out-of-the-box in real experiments, closure tests are fundamental tools to demonstrate that a given data-driven method to measure calibration factors or efficiencies works as advertised, and without biases outside of the quoted uncertainties. In other words, a method not closing indicates the need to go back to the drawing board and understand the biases in the measurement procedure that are responsible for the lack of closure. Closure tests are only useful if the simulated samples accurately model the details of real data because, otherwise, some effects from the measuring procedure may be missed. The basic principle of closure tests is the possibility to compare MC truth values to data-driven measurements, with the former calculated directly from detector-level-to-particle-level information, and the latter derived from methods applied to detector-level-only information. For example, the MC truth jet energy response is determined from the ratio between the energy of detector-level reconstructed jets and the MC truth jet energy calculated as the sum of energies of all the final state particles identified as part of the jet before they hit the detector. If the jet energy response measured from detector-level MC information using data-driven methods, such as $\gamma$-jet and di-jet balance, is consistent within uncertainties with the MC truth jet energy response, then the method closes. Typically, closure tests take the form of detector-level-toparticle-level ratios for an observable of interest as a function of variables such as transverse momentum or pseudorapidity. For a method to close, the ratio has to be consistent with unity within the quoted uncertainties.

The lack of high-quality, high statistics MC samples in the D0 experiment was one of the causes of delay in the publication of a number of physics measurements. For example, the D0 Run 1 jet papers to validate QCD predictions were only published in the late 1990's and early 2000's, once the jet energy was calibrated to a $\sim 3 \%$ accuracy level. The challenge to uncover the biases of the data-driven methods used to measure the jet energy scale was at the core of the publication delay. The difficulty arose from the lack of MC samples that modeled response linearity and shower shapes to the necessary level of accuracy.

The concept of closure test is illustrated in Fig. 18, which demonstrate the di-jet balance and bisector methods to measure jet energy resolutions in ATLAS [39. The MC truth resolution is shown in full circles, and the measured 
resolutions extracted from MC detector level samples using the di-jet balance and bisector techniques are shown in open squares and circles respectively. The lower panel demonstrates a better than $10 \%$ accuracy level and indicates the methods are biased to slightly overestimate the resolutions.

For the same CMS muti-jets SUSY final state introduced in Sec. 4.1.1. Figs. 19, 20 illustrate $\mathrm{MC}$ closure tests of a data-driven method to predict the SM background coming from $t \bar{t}+$ jets and $W+$ jets events [47. The background arises from events that mistakenly pass the $p_{T}^{\text {lep }}>10 \mathrm{GeV}$ veto cut on the $p_{T}$ of isolated leptons, introduced to remove events with high $p_{T}$ isolated leptons from the signal sample. These mistakes occur when the lepton escapes detection due reconstruction and identification inefficiencies. The data-driven method is based on the selection of a one lepton plus jets control sample by inverting the lepton veto requirement. In such a sample, $97 \%$ of the events are either $t \bar{t}+$ jets or $W+$ jets. Once the number of events in the control region is normalized by a factor accounting for reconstruction and identification efficiencies, $\left(1 / \varepsilon_{\text {iso }}\right)\left[\left(1-\varepsilon_{\text {iso }}\right) / \varepsilon_{\text {reco }+ \text { id }}\right]$, and the sample is restricted to the signal region, also referred to as the search region, the control sample predicts the number of electroweak background events in the search region. Figs. 19,20 show a comparison between the predicted background (circles) and the MC truth estimated background (histograms) for $H_{T}$ (scalar sum of the jet $p_{T}$ 's), $H_{T}^{\text {miss }}$, and jet multiplicity. The excellent closure within statistical uncertainties for all three observables indicates that the method predicts the background with high accuracy and any potential biases are under control, within the quoted uncertainties. Had there been deviations of the ratios from unity, outside statistical and systematic uncertainties, potential sources of biases on the method would have been further investigated and eventually removed, most probably at the cost of additional systematic uncertainties.

\subsection{Simulation in Detector Design and Optimization}

HEP collider detectors consist of devises based on diverse technologies specialized in observing and characterizing the different types of particles that result from high-energy collisions. A typical HEP collider detector includes tracking modules to measure the interaction vertices and the tracks of charged particles, calorimeters to measure energy depositions as a result of electromagnetic and hadronic showers, wire chambers to detect high energy muons, and magnets for particle identification and momentum measurement. For event reconstruction, modern experiments follow a holistic approach, using all sub-detector components to reconstruct each particle individually by means of complex software algorithms. This is the case of the particle flow technique used by CMS [37.

To design a HEP detector, different technologies and physical characteristics are modeled and optimized in simulation for best physics performance. For example, the efficiency and precision of particle tracking algorithms in a silicon detector typically improves by increasing the pixel and strip density, the number of layers, and the angular coverage, as well as by minimizing the amount of material a particle traverses. Muon detection improves with the wire chamber 


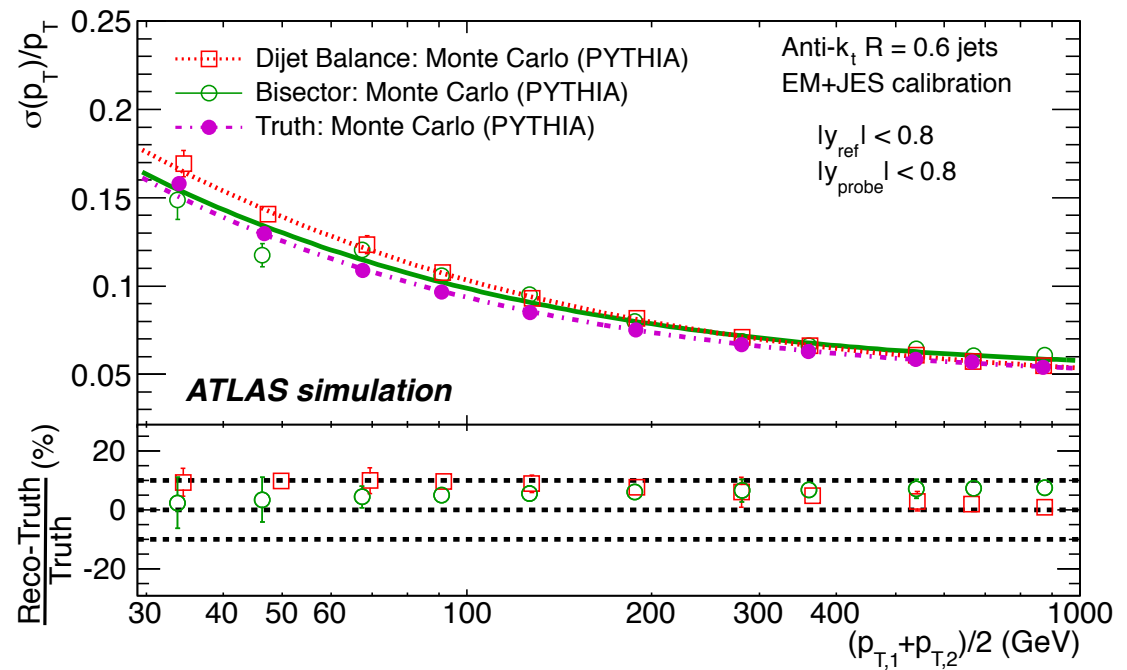

Figure 18: Illustration of a closure test 39. Comparison between the MC truth jet $p_{T}$ resolution and the results obtained from the bisector and di-jet balance methods applied to detector level MC as if it were data. The lower panel shows the percentage difference, obtained from the fits. The errors shown are only statistical. 

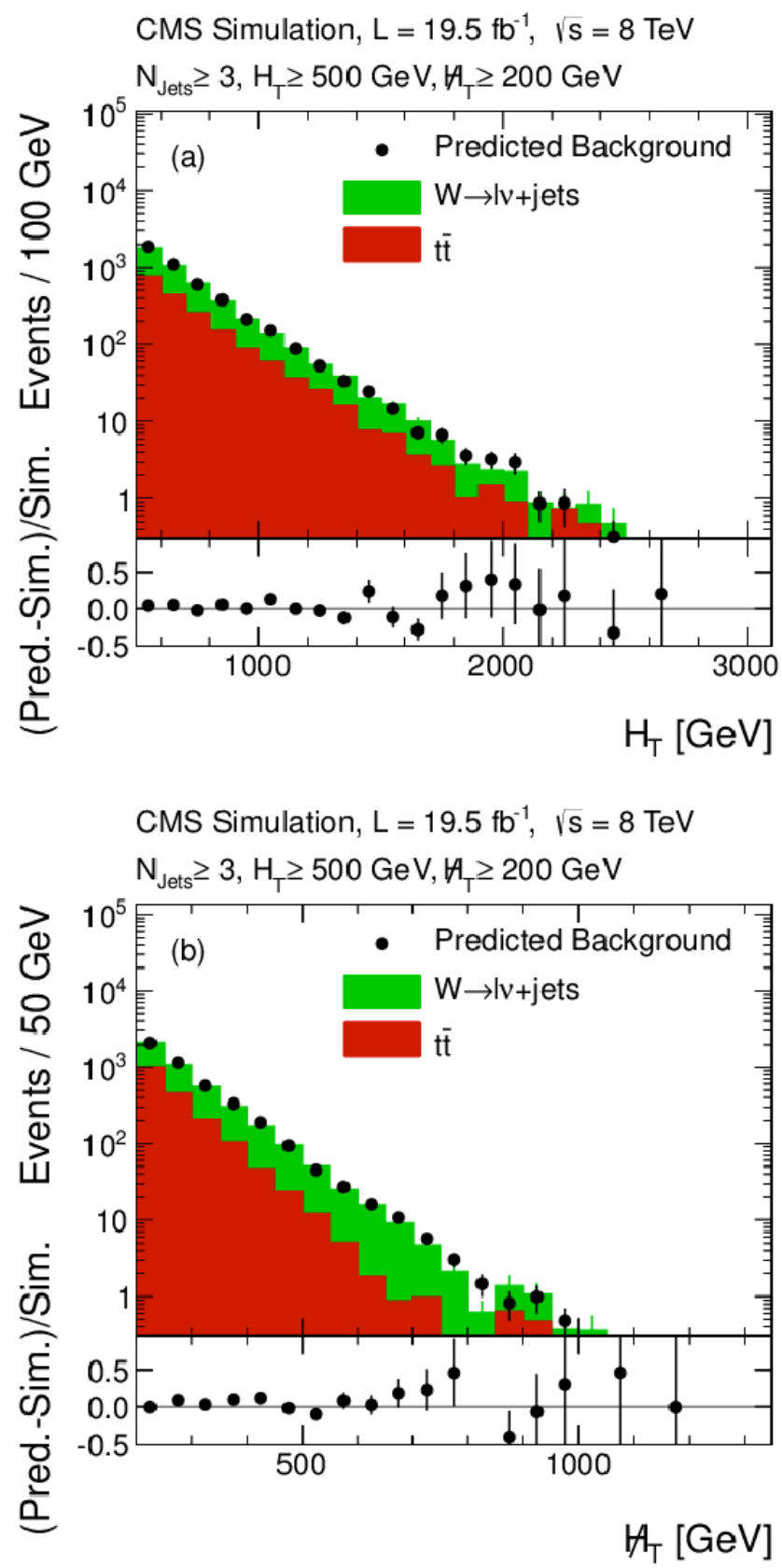

Figure 19: Illustration of a closure test. Data-driven $t \bar{t}+$ jet and $W+$ jets backgrounds (circles) are compared with the MC truth estimate (histograms) for the $H_{T}$ and $H_{T}^{\text {miss }}$ observables in a CMS multi-jets SUSY search [7]. 


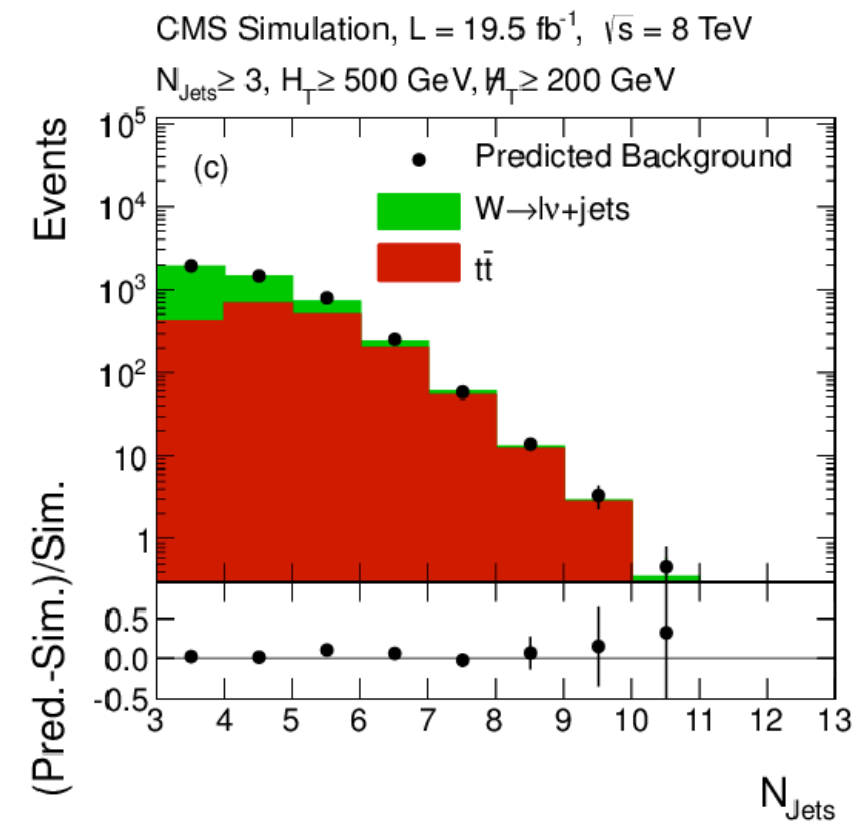

Figure 20: Illustration of a closure test. Data-driven $t \bar{t}+$ jet and $W+$ jets backgrounds (circles) are compared with the MC truth estimation (histograms) for the jet multiplicity distribution in a CMS multi-jets SUSY search 47. 
density, number of layers in the radial direction, and angular coverage. More powerful or weaker magnets allow for more compact or larger designs, with a range of momentum resolutions. An ideal calorimeter provides full solid angle coverage and hermeticity, and improves its performance with higher transverse granularity, longitudinal segmentation, and materials that yield Gaussian and narrow response functions. These parameters are varied in the simulation and the final design is selected using a cost-benefit equation that considers monetary cost versus detector physics performance.

Monte Carlo simulation campaigns for detector design and optimization produce millions of events generated with different detector scenarios that vary in technology options and physical parameters. Goals range from making a case for a given detector configuration, to optimizing a design for maximum physics output, or investigating the physics impact of detector de-scoping options driven by budgetary constraints. Nowadays, these simulation efforts are an absolute requirement for every HEP experiment seeking approval from funding agencies. Interestingly, Geant4 plays a dual role in the process. Firstly, it helps select the optimal design that does the physics job for the available budget. Secondly, Geant4 influences the detector design by adding its own software and computing constraints to the decision process. In other words, the optimal geometric designs are often too difficult to model with Geant 4 or very expensive in computing resources. While Geant 4 evolves to support experiments with more features and speed, detector configurations also adapt to play to the strengths of the Geant4 simulation toolkit.

Figs. 21, 22 illustrate the use of simulation for design studies in the CMS experiment [48]. Performance tests evaluate basic detector level observables, such as track efficiencies, photon, electron, muon and jet resolutions, as well as the potential precision of cross section or mass measurements, or the discovery reach for new particles. As an example, Fig. 21 (top) shows the predicted CMS tracking efficiency versus pseudorapidity for various tracker design options and accelerator performance parameter values associated with the high-luminosity LHC (HL-LHC) run scheduled to start in 2026. Efficiency is studied for different accelerator performance scenarios, expressed in terms of instantaneous luminosity, $L=\frac{1}{\sigma} \frac{d N}{d t}$, where $d N / d t$ is the number of events produced as the result of the hard collision and $\sigma$ is the interaction cross section. $L$ depends on detector parameters such as the number of particles in a bunch within the beam, and the size of the beams. At the LHC, as $L$ increases, the probability of multiple proton-proton ( $\mathrm{pp}$ ) interactions per crossing with low momentum transfer increases. These spurious interactions pile-up and overlap with the high- $p_{T}$ event of interest which fired the physics trigger. For different pile-up (PU) scenarios, measured in terms of the number of spurious pp interactions, Fig. 21 (top) shows the tracking efficiency for the 2017 detector (Phase I detector, black squares) and the proposed 2026 detector (Phase II detector, blue, red, green symbols) with and without a tracker upgrade that would extend the $\eta$ coverage to 3.8. The addition would extend the coverage in the region near the beam pipe, improving the tracking efficiency and reducing the fake rate (not shown), thus allowing to suppress more efficiently any spurious contribution 
from pile-up events to the high- $p_{T}$ event under study. Fig. 21 (bottom) shows the relative degradation in photon energy resolution, measured in gluon fusion Higgs events, as a function of the number of layers removed from the proposed CMS endcap calorimeter. $N_{b / a}$ is the number of layers before and after removal. This degradation affects measurements with the Higgs boson decaying to two photons or four electrons.

Fig. 22 (top) shows how much energy from pile-up events contribute on average to a reconstructed jet as the number of pile-up events increases with luminosity. This spurious contribution changes the jet multiplicity of the event, distorts the jet energy response, and degrades jet energy and missing transverse energy resolutions. Missing transverse energy is a measure of the event momentum imbalance in the transverse plane and will be defined and discussed properly in Sec. 5.2.3. Fig. 22 (bottom) shows, using the Delphes parametrized simulation framework [49, the impact of the tracker extension and the number of pile-up events on the sensitivity of the proposed detector to SUSY particle production. The study investigates a model with electroweak production of a chargino-neutralino pair, $\tilde{\chi}_{1}^{ \pm} \tilde{\chi}_{2}^{0}$, decaying to $W H$ and two stable neutralinos, $\tilde{\chi}_{1}^{0}$, the latter being the lightest stable particle (LSP) predicted by the model. For an integrated luminosity of $3000 \mathrm{fb}^{-1}$, that is the integral of the instantaneous luminosity over the time covering the full data set expected to be collected by the CMS experiment during the HL-LHC run, the sensitivity is explored for three different scenarios: $140 \mathrm{PU}$ events with and without a tracker extension, and $200 \mathrm{PU}$ events with a tracker extension. The limits on the chargino mass are very sensitive to an increase in the number of PU events, although a fraction of the sensitivity is recovered with the tracker extension.

The ATLAS Collaboration also performed various MC studies to optimize its detector design for the HL-LHC era. Ref. 50 contains a description of the upgrade options under consideration, which include extensions to the tracker and pixel detectors to cover pseudorapidity ranges of $|\eta|<4$ (Reference scenario), $|\eta|<3.2$ (Middle scenario), or $|\eta|<2.7$ (Low scenario). The upgrade also includes improvements to the trigger system, detector electronics, and forward calorimetry (Reference scenario). The results presented here are based on simulated events produced with the ATLAS Geant4-based simulation application. Fig. 23 (top) shows the momentum dependence of the muon reconstruction plus identification efficiency for different ATLAS detector upgrade scenarios and $200 \mathrm{PU}$ events. It is apparent that the detector descoping from the Reference scenario to the Low scenario would cost the experiment $10 \%$ in muon efficiency. The primary vertex reconstruction efficiency in ATLAS for $t \bar{t}, Z \rightarrow \mu^{+} \mu^{-}$, and Vector Boson Fusion (VBF) $H \rightarrow \gamma \gamma$ events is shown in Fig. 23 (bottom) in the case of $200 \mathrm{PU}$ events. The lesson learned is that vertexing performance does not depend strongly on the tracker layout, and it varies with physics process. While the efficiency does not change for $t \bar{t}$, it goes down by $1 \%(2 \%)$ for VBF $H \rightarrow \gamma \gamma\left(Z \rightarrow \mu^{+} \mu^{-}\right)$events when switching from the Reference to the Low scenario. Fig. 24 (top) illustrates on the reduction in the photon conversion cumulative probability as a function of the distance from the interaction vertex (radius) when the ATLAS Inner Tracker (ITk) is upgraded. The ATLAS SUSY 

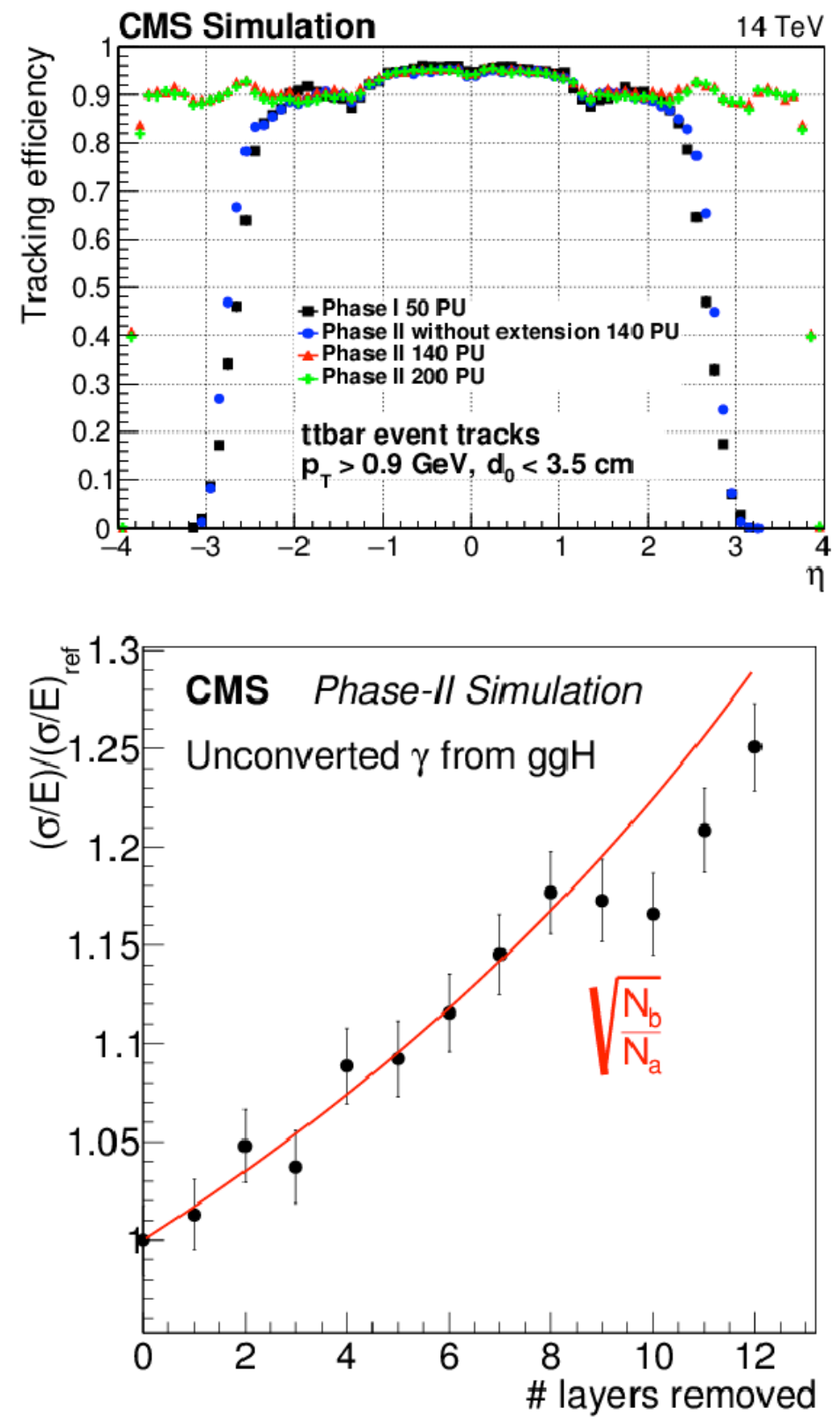

Figure 21: Top: Predicted CMS tracking efficiency versus $\eta$ for a range of luminosity scenarios (number of pile-up events), and two detector upgrade options, with and without extended $\eta$ coverage [4]. Bottom: Relative degradation in photon energy resolution in gluon fusion Higgs events as a function of the number of layers removed from the proposed CMS endcap calorimeter [48]. 

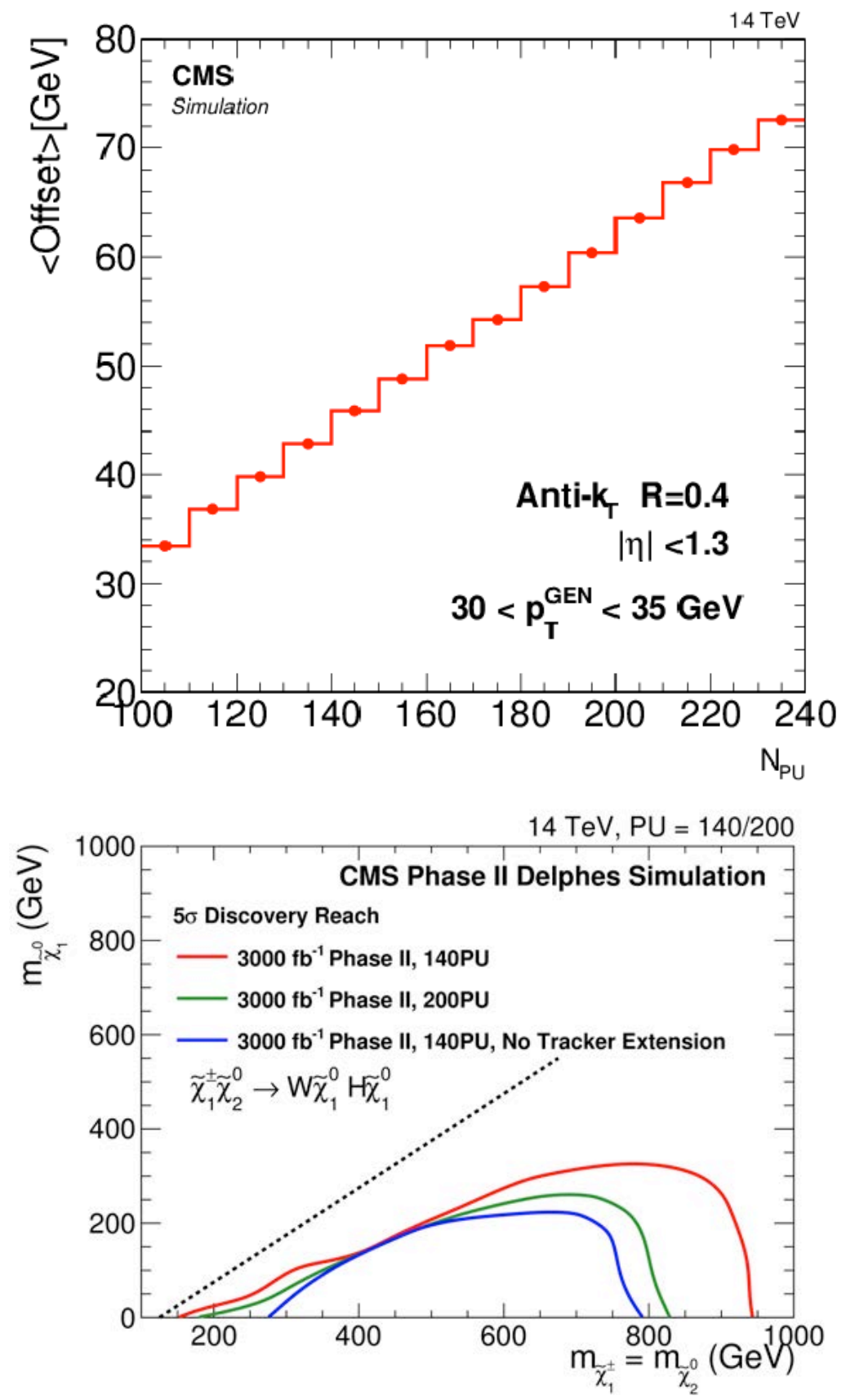

Figure 22: Top: Average energy from pile-up events contributing to a reconstructed jet as the number of pile-up events increases with luminosity [48. Bottom: Effect of luminosity and impact of a proposed CMS tracker extension in the sensitivity to SUSY particle production [48, 49. 
search shown in Fig. 24 (bottom) is for the production of a chargino-neutralino pair $\tilde{\chi}_{1}^{ \pm} \tilde{\chi}_{2}^{0}$, which decays into a $W$, a SM-like Higgs boson, and LSP (neutralinos). The final state consists of two jets, one isolated lepton, two b-jets, and large missing transverse momentum coming from the weakly interacting neutralino, $\tilde{\chi}_{1}^{0}$. The study is performed for $200 \mathrm{PU}$ events and show a $200 \mathrm{GeV}$ improvement in the limit to the chargino/neutralino mass for low LPS masses, when switching from the Low to the Reference detector scenario.

\subsection{Simulation in Software and Computing Design and Testing}

Simulation is also an essential tool to develop each element of the workflow and dataflow associated with data handling in large HEP experiments. At the LHC, the Worldwide LHC Computing Grid (WLCG) [51 is used to process, store and analyze the data collected or generated by the experiments. The WLCG is composed of four levels or "Tiers": $0,1,2,3$. The difference between Tiers is in the services they provide, whether they host raw data, and how well they are interconnected. The Tier 0 is located at CERN in Geneva, Switzerland and at the Wigner Research Centre for Physics in Budapest, Hungary. All data passes through the two Tier 0 sites, which are connected by two dedicated $100 \mathrm{Gbit} / \mathrm{s}$ data links and provide less than $20 \%$ of the compute capacity. The main role of the Tier 0 is to safe-keep the raw data, perform a first pass reconstruction, reprocess data when the LHC is not running, and distribute the raw and reconstructed data to the Tier 1 centers. The Tier 1 consists of 13 computing centers with large storage capacity distributed all over the world. They are responsible for the safe-keeping of different shares of all raw and reconstructed data, as well as for performing large-scale reprocessing and storing the associated output. The Tier 1 centers distribute data to the Tier 2 centers and store a share of the simulation output produced by the Tier 2's. A dedicated high-bandwidth network, consisting of $10 \mathrm{Gbit} / \mathrm{s}$ optical-fiber links, connect CERN to most of the Tier 1 centers around the world. The approximately 160 Tier 2 centers are typically located at research institutions outside CERN and provide data storage capacity and computing power for simulated event production and reconstruction, as well as for data analysis tasks. Tier 3 computing resources are not part of the WLCG and refer to local clusters in universities, other scientific institutes, or even individual PC's, that scientists use to access the WLCG resources.

In CMS, the combined procedure of data acquisition, processing, transfer, and storage using WLCG resources was tested in a series of computing, software and analysis (CSA) challenges. The tests included components such as the preparation of large simulated data-sets, prompt reconstruction at the Tier 0 center, the distribution of output files to Tier 1 centers for re-reconstruction and skimming, calibration jobs on alignment and calibration data-sets, and physics analysis in Tier 2 centers. In a series of exercises in 2006, 2007, 2008 (Run 1) and 2014 (Run 2), the computing system was stress tested at 25\%, 50\%, and $100 \%$ capacity. In preparation for Run 1, 150 million simulated events were produced, realistic trigger rates were modeled, and reconstruction and physics analysis

performed in real time for event samples representing an integrated luminosity 

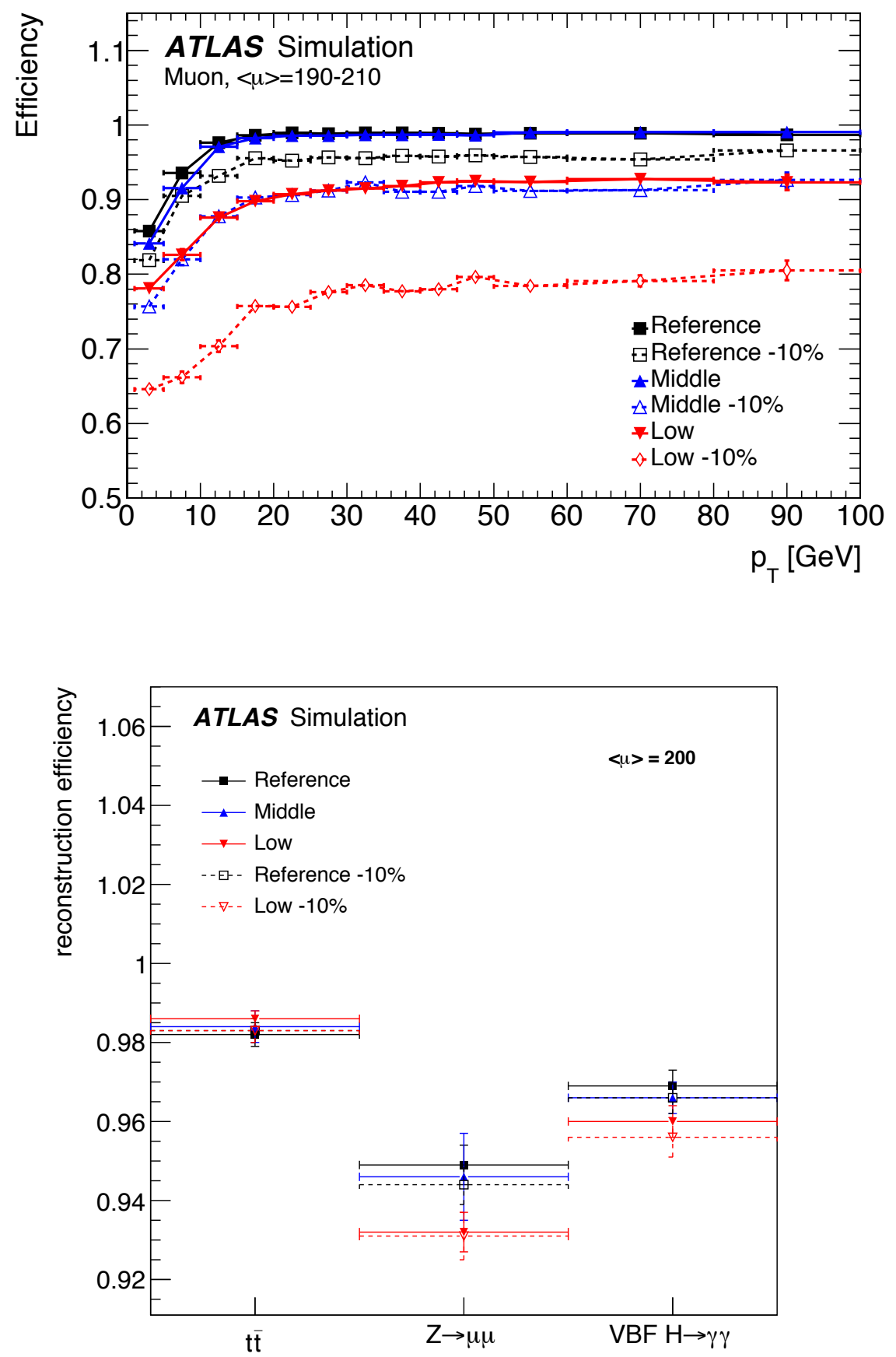

Figure 23: Top: Momentum dependence of the muon reconstruction plus identification efficiency for different ATLAS detector upgrade scenarios and 200 PU events [50]. Bottom: Primary vertex reconstruction efficiency in $t \bar{t}, Z \rightarrow \mu^{+} \mu^{-}$, and Vector Boson Fusion (VBF) $H \rightarrow \gamma \gamma$ events for the three ATLAS detector upgrade scenarios and 200 PU events [50]. 

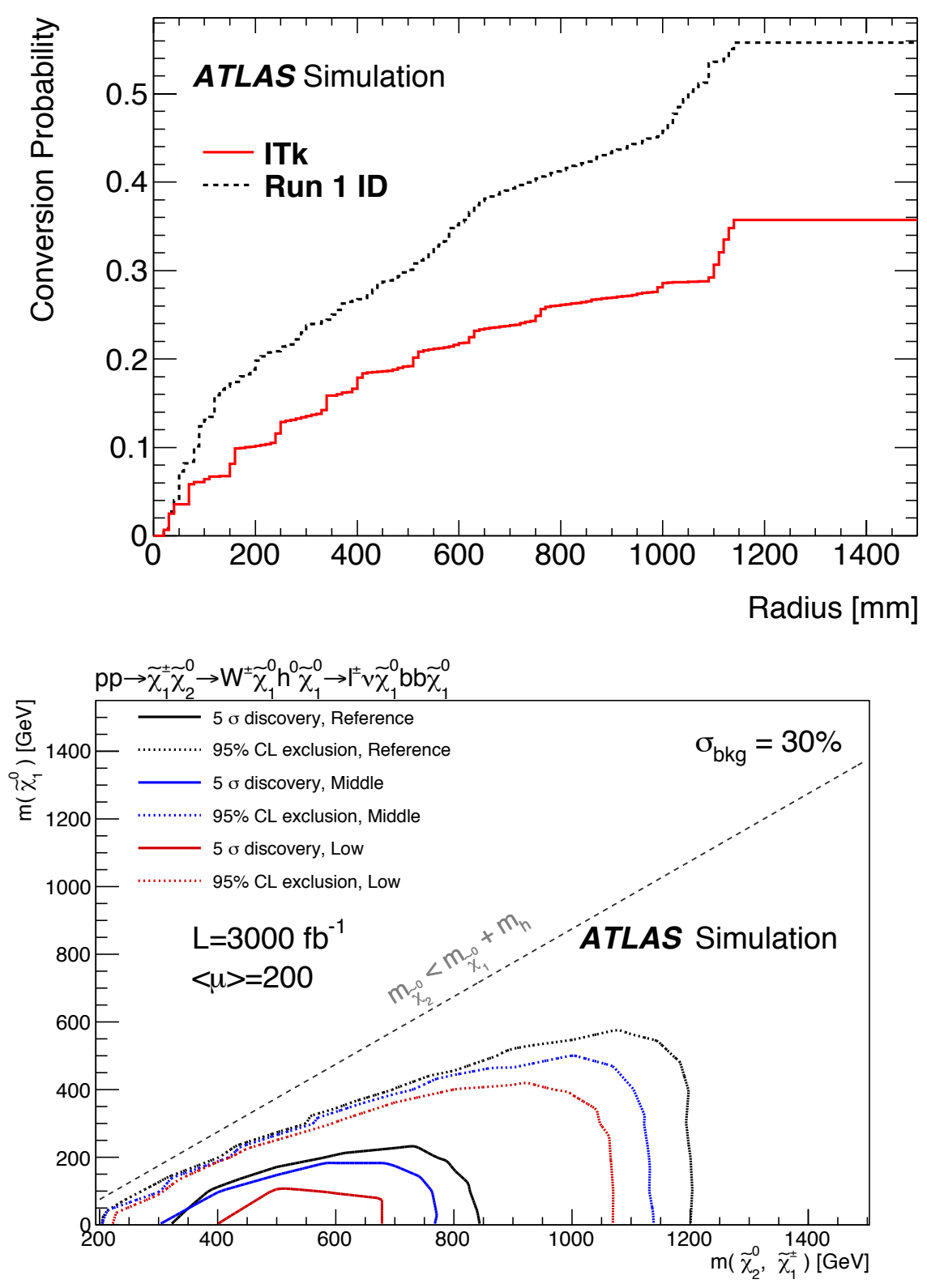

Figure 24: Top: Photon conversion cumulative probability as a function of the distance from the interaction vertex (radius) for the Run 1 and HL-LHC ATLAS detectors [50]. Bottom: Sensitivity to the different detector upgrade scenarios of an ATLAS SUSY search for the production of a chargino-neutralino pair $\tilde{\chi}_{1}^{ \pm} \tilde{\chi}_{2}^{0}$ in the case of $200 \mathrm{PU}$ events [50]. 
in excess of a quarter of the total delivered in 2010. For illustration, the workflow for the CMS 2008 CSA challenge 52 is shown in Fig. 25. The "pre-production" samples are simulated data modeling the real raw data acquired by the detector and filtered by the trigger according to the same physics requirements coded in the actual trigger system. This step was performed in various Tier 0,1 and 2 computing centers, and the resulting output data copied to the Tier 0 center at CERN, where prompt reconstruction followed. Next, the MC data-sets utilized for calibration and alignment, the "AlCaReco" files, were produced and transferred to the CERN Analysis Facility (CAF). Then, the calibration and alignment constants were derived at the CAF and transferred to the conditions database. The data was reprocessed in the Tier 1 center, re-reconstructed as is typical in the experiments to correct mistakes in the first pass. Finally, the physics analysis was performed in the Tier 2 centers.

The realism of these rehearsals in $21^{\text {st }}$ century experiments has allowed them to reach data taking with an unprecedented degree of preparedness. Event and file sizes, memory and CPU time consumption, detector geometry description and alignment, particle showering in the detector material, electronics, calibration procedures, prompt reconstruction, data transfer between computing processing centers were tested so accurately and realistically with MC samples, that experiments as complex as ATLAS and CMS did not meet major surprises during start-up, with most components working as predicted, within design specifications and, basically, out of the box.

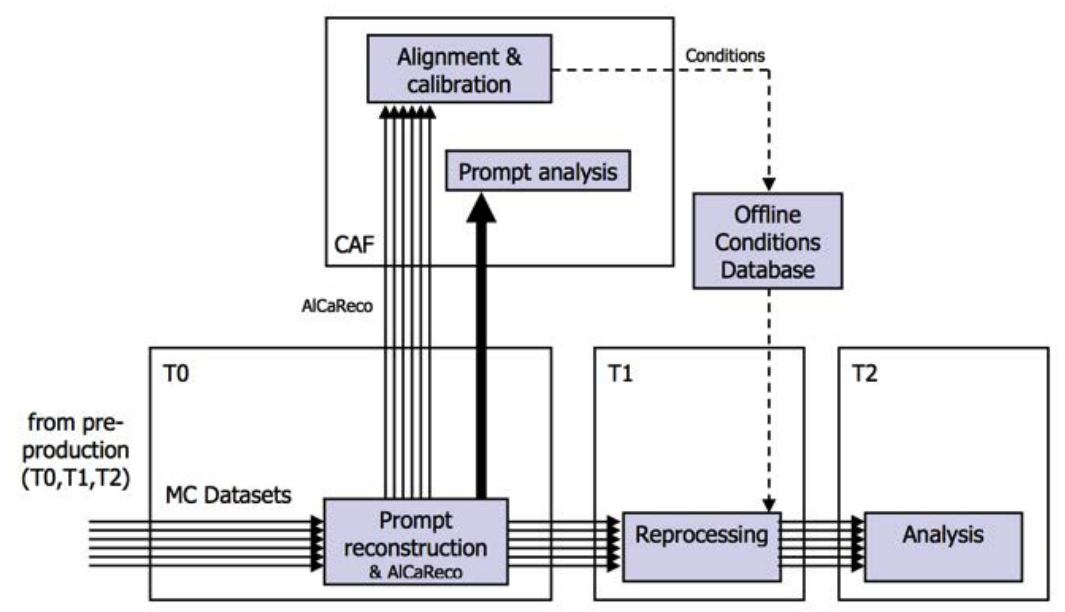

Figure 25: Workflow for the CMS 2008 Software, Computing, and Analysis challenge (CSA) [52]. 


\section{Simulation of Collider Physics Observables for Particles and Events}

The level of agreement between the MC predictions of physics observables and the corresponding data measurements are a test of the accuracy of the simulation software. This section starts with a discussion on the impact of the detector geometry and materials modeling on the simulation of photons, electrons, and muons. It follows with data-to-MC comparisons for b jet identification variables, a set of $W / Z+$ jets observables, and missing transverse energy

distributions and resolutions. The impact of simulation in the precision of jet cross section measurements and publication timeline is presented at the end as a case study.

\subsection{Geometry and Material Modeling Effects on Photon, Electron, and Muon Simulation}

Accurate simulation of electrons and photons necessitates a very detailed description of the material and thickness of the tracker system components. Typically, trackers are highly segmented to provide efficient particle identification and precise measurements of particle trajectories and momentum in the presence of a magnetic field. In addition, these detectors must be thin and light to minimize interactions before the particles reach the calorimeters. In an ideal detector, electrons, photons, and hadrons would traverse the tracker unperturbed and experience their first destructive interaction and subsequent shower in the calorimeters, the detector components designed to measure energy. In real detectors, most significantly in the case of silicon trackers, particles do interact and disappear (photon conversion) or loose a large fraction of their total energy while traversing the detector material (charged particles). This is a price that most modern experiments are willing to pay in exchange for the more precise position and momentum measurements, faster readout, and better radiation tolerance offered by silicon-based detectors. For example in CMS, photons have a $70 \%$ probability to convert into electrons within the silicon tracker volume, a difficult challenge to overcome given the key role that photons play in Higgs measurements $(H \rightarrow \gamma \gamma)$, direct photon strong production studies, and BSM searches. Simulation is a useful tool to understand the impact of tracking detector material on physics measurements, keep the systematic uncertainties under control, and deliver competitive results. The necessary condition is that the tracker materials, shapes, and thicknesses are described with precision in the geometry code, and that photon-nucleus interactions, photon conversions and energy loss, as well as multiple scattering are accurately modeled in Geant4.

In the CMS simulation software, the implementation of the shapes and materials of the tracker geometry elements (350,000 volumes) was followed by careful validation to achieve accuracy. Fig. 26 shows the total thickness of the CMS tracker material in units of radiation lengths $X_{0}$ (top) and interaction lengths $\lambda_{I}$ (bottom) that a particle produced at the center of the detector would traverse as it moves along different pseudorapidity directions in the $\eta<2.5$ acceptance region. The contribution to the total material of each of the subsystems that comprise the CMS tracker is given separately: the pixel tracker, the strip tracker 
which consists of the tracker endcap (TEC), the tracker outer barrel (TOB), the tracker inner barrel (TIB), and the tracker inner disks (TID), the support tube that surrounds the tracker, and the beam pipe [53. Fig. 27] presents the data-to-MC ratio of the fraction of photons undergoing conversions and nuclear interactions as a function of the radial distance $(R)$ from the center of the detector, which is correlated with different sub-detector components. This ratio is computed from data-driven measurements of the conversion and nuclear interaction probabilities respectively, demonstrate agreement between data and $\mathrm{MC}$ within $15 \%$, and may be used as scaling factors to correct the MC before using it in physics analysis. Discrepancies observed in Fig. 27 can be directly related to deficiencies in the detector geometry modeling [54].

Muons are also particularly sensitive to the modeling of the detector geometry and material, because they interact very little with matter, and therefore traverse all detector sub-systems in a collider experiment. Fig. 28 shows the $q \times p_{T}$ and $\eta$ distributions, where $q$ is the muon charge, for CMS muons selected from zero-bias data 43 . Zero-bias refers to a sample of events collected from random proton bunch crossings without any specific trigger requirement. The sub-sample of all muons contained in the zero-bias sample includes the contributions of prompt muons from $W$ and $Z$ decays, muons from heavy flavor decays (b- and c-quarks or $\tau$-leptons), light hadrons $(\pi, K)$ or decays of particles produced in nuclear interactions, and muons from hadrons that penetrate the detector beyond the limits of the calorimeters. In Fig. 28, the inclusive muon sample selected in data is compared with the sum of the MC predictions for each of the above-mentioned processes. The excellent agreement in the kinematic regions where data and $\mathrm{MC}$ are compared, $p_{T}=1-20 \mathrm{GeV}$ and $|\eta|<2.6$, is remarkable given that the pixel, tracker, and muon systems are all used in muon reconstruction, involving a diversity of technologies, shapes, and materials, as well as abrupt transitions between sub-detector systems.

As in the case of CMS, the ATLAS detector also includes a silicon-based inner detector for vertex and track reconstruction, which extends to a radius of $1.15 \mathrm{~m}$, and is $7 \mathrm{~m}$ in length along the beam pipe. The development and validation of simulation code to model the detector shape, thickness, and materials was therefore an activity of utmost importance. Fig. 29 (top) shows the distribution of photon conversion vertices in the radial direction starting from the detector center [55. Full circles represent the collider data measurement, while the solid line shows the distribution of conversion candidates obtained using the same analysis method applied to the data. The histogram shows the true MC distribution for the conversions (blue) and the Dalitz decays of neutral mesons (yellow). The good agreement between MC and data, although based on limited statistics, is a measure of the excellent modeling of the material distribution in the MC. A second example of material modeling validation is shown in Fig. 29 (bottom) [56] and consists of reconstructing particles with well known masses and lifetimes from detector tracks. Flaws in the material modeling of the detector would result in incorrect compensation for effects of energy loss and multiple scattering on the tracks, resulting in biases to the reconstructed tracks momenta, which propagate to the reconstructed mass. In the case of the $K_{s}^{0}$, 

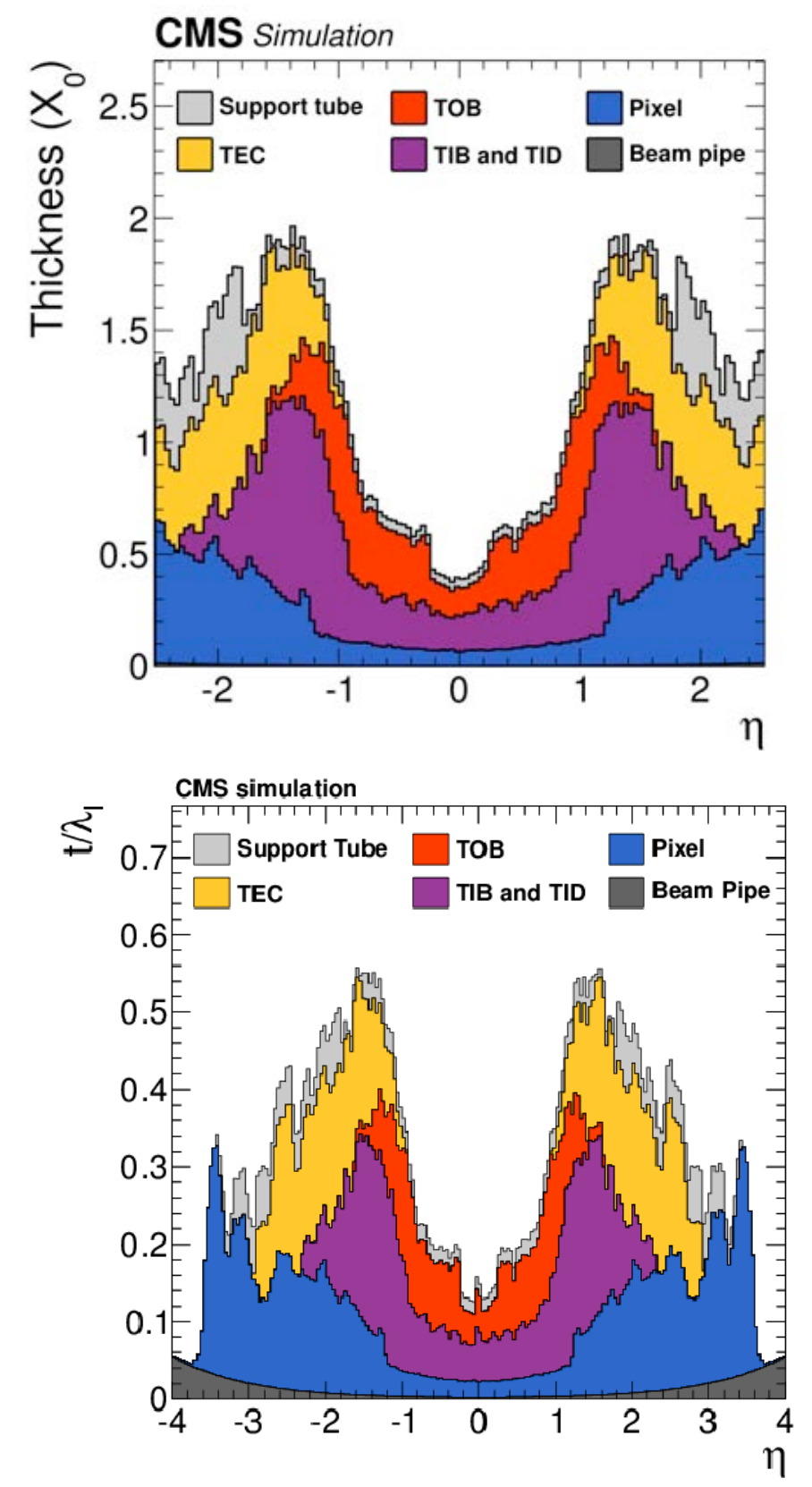

Figure 26: Total thickness of the CMS tracker material, in units of radiation lengths $X_{0}$ (top) and interaction lengths $\lambda_{I}$ (bottom), that a particle produced at the center of the detector would traverse as it moves along different pseudorapidity directions in the $\eta<2.5$ acceptance region. The contribution to the total material of each of the subsystems that comprise the CMS tracker is shown separately. 


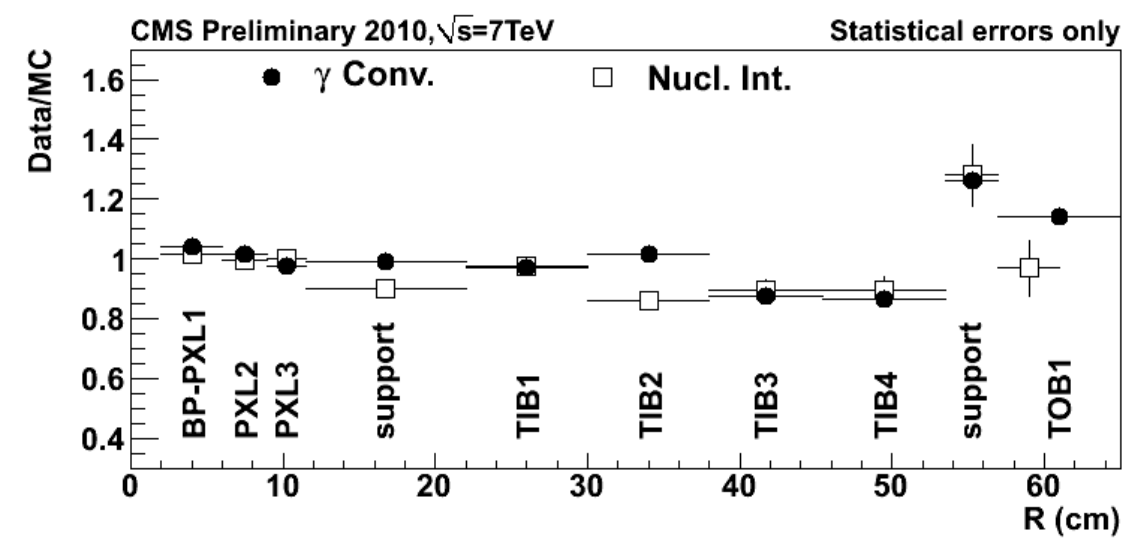

Figure 27: Data-to-MC ratio of the fraction of photons undergoing conversions (full circles) and nuclear interactions (open circles) in the CMS tracker volume. Discrepancies between $\mathrm{MC}$ and data can be related directly to deficiencies in the detector geometry modeling of the tracker [54]. The ratio is plotted as a function of the radial distance $(R)$ from the center of the detector, which is correlated with different sub-detector components.

which decays with a proper length of $c \tau \sim 2.7 \mathrm{~cm}$, it is possible to study the detector material modeling accuracy as a function of the radial position of the decay vertex. The sample used in the study consists of a selection of oppositely charged track pairs with $p_{T}>100 \mathrm{MeV} . K_{s}^{0}$ candidates were reconstructed with a fit to the pairs satisfying a selection criteria. Fig. 29 (bottom) shows the data-to-MC ratio of the measured $K_{s}^{0}$ mass as a function of the radial distance to the center of the detector, with dashed lines marking the boundaries of the sub-detector systems. Once again, the high level of agreement of the fitted $K_{s}^{0}$ in $\mathrm{MC}$ and data is a measure of the excellent modeling of the ATLAS silicon tracker material distribution and thickness in the simulation.

\subsection{Modeling of Particle and Event Properties and Kinematics}

This section includes a number of data-to-MC comparisons from ATLAS and CMS focused on event or particle properties and kinematics. Examples are presented for photons, electrons, muons, and jets from light and heavy quarks. These particles are observed and reconstructed as physics objects in the detector, and constitute the basic ingredients of every measurement. Excellent understanding of their kinematic distributions, as well as their reconstruction and identification efficiencies is a first step for any experiment to deliver robust physics measurements of high quality and precision.

\subsubsection{Tagging of Heavy Quarks}

The ability to model b-jet reconstruction and identification is an important simulation benchmark. In hadron colliders, the identification of jets originating from b quarks is critical for both SM measurements and BSM searches, given 

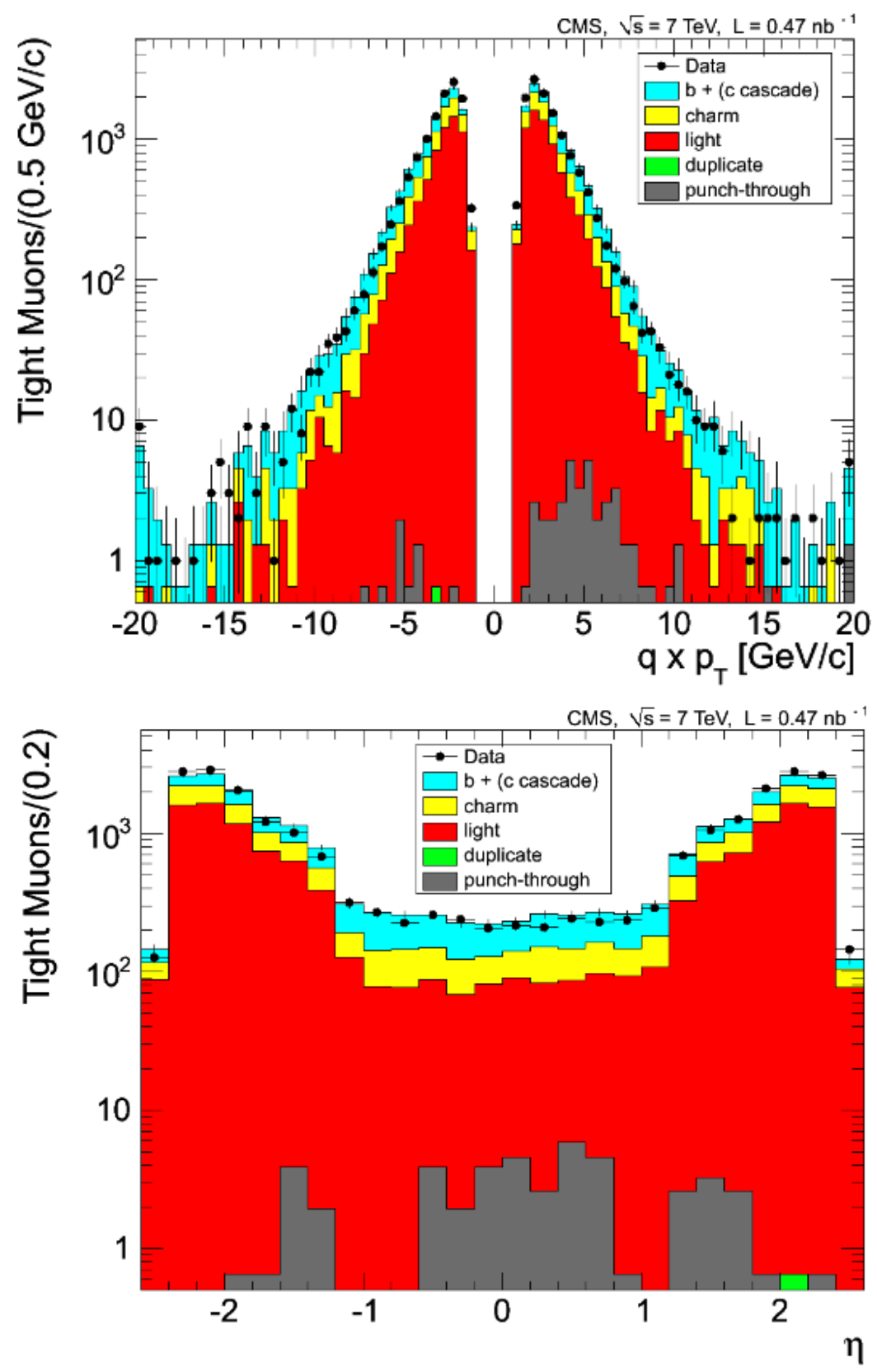

Figure 28: Muon $q \times p_{T}$ ( $q$ is the charge) and $\eta$ distributions for a CMS sample of muons selected by the zero-bias trigger. Data is presented in full circles and simulation in histograms [43. The error bars indicate the statistical uncertainty. 

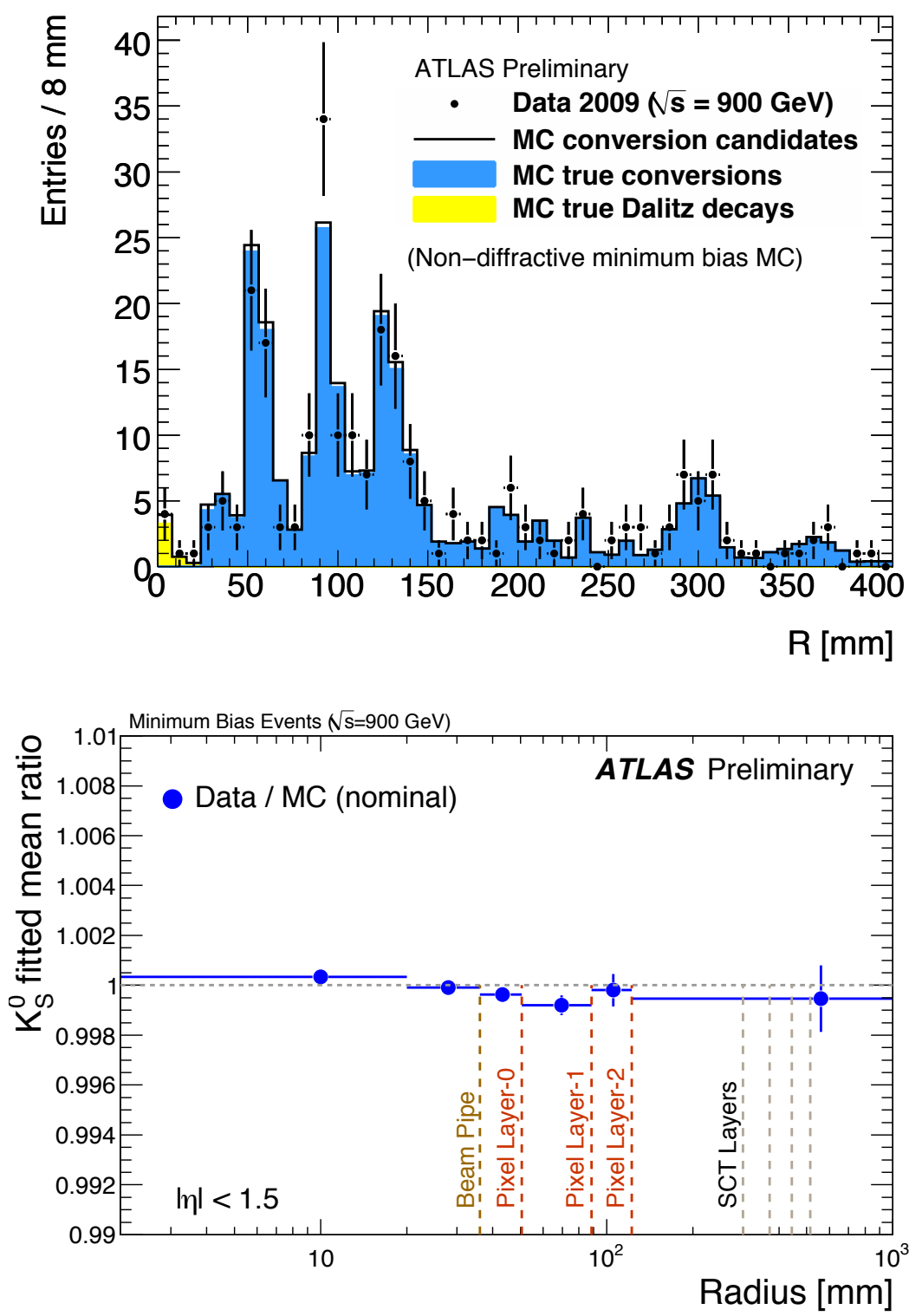

Figure 29: Top: ATLAS distributions of photon conversion vertices in the radial direction starting from the detector center are shown for collider data, conversion candidates, obtained using the same analysis method applied to the data, true photon conversions, and true Dalitz decays of neutral mesons [55]. Bottom: Data-to-MC ratio of the ATLAS measured $K_{s}^{0}$ mass as a function of the radial distance to the center of the detector, with dashed lines marking the boundaries of the sub-detector systems [56. 
that top quarks decay into a b jet and a $W$ boson, and flavor is intimately tied with the Electroweak Symmetry Breaking mechanism (EWSB). Furthermore, SUSY and EWSB are related via the hierarchy problem. Thus b-jet identification is a key component of the event selection criteria developed for BSM searches, and accurate modeling of b-jets and b-tagging related variables are essential to understand data selection efficiencies and simulate the signal samples.

The b-jet identification procedure, or b-tagging [57, depends on variables and requirements such as the impact parameters of charged-particle tracks in a jet, the properties of reconstructed decay vertices in the jet, and the presence or absence of a lepton within a jet. The 3-Dimensional Impact Parameter (3D IP) is defined as the point of closest approach between a track and the event primary vertex $(\mathrm{PV})$. The impact parameter has the same sign as the scalar product of the vector pointing from the primary vertex to the point of closest approach with the jet direction. In an ideal detector, tracks originating from the decay of long-lived particles such as b quarks traveling along the jet axis would have positive IP values, while the impact parameters of light-flavor quarks coming from the PV would be still be positive but close to zero. However, in a real detector, both negative and positive values are possible due to resolution effects. While distributions are significantly asymmetric for b-quarks, they are almost symmetric for light quarks, with a deviation towards a small positive mean value due to contributions of secondary vertices from particles decaying within the light jets, such as kaons and lambdas. Fig. 30 (top) shows two tracks originating from the secondary vertex (SV) and bending outwards due to the effect of the solenoidal magnetic field. The impact parameter is indicated as the distance between the primary vertex (PV) and the back-propagated tracks. Fig. 30 (bottom) illustrates the sign convention for the impact parameter.

The 3D IP distribution for tracks in jets selected in a CMS di-jet trigger sample is presented in Fig. 31 [57. Data is compared with MC predictions for all the parton flavors contributing to the inclusive di-jet sample. As expected, while the distributions for tracks in heavy-flavor jets are significantly asymmetric with a positive mean value, the distribution for light quarks and gluons is almost symmetric. The excellent agreement between 3D IP distributions in data and MC, within less than $10 \%$, is a precondition to the development of accurate data driven methods to measure b-tagging efficiencies. These efficiencies, shown in Fig. 32, are derived from a sample of jets with muons for two different b-tagging algorithms, known by their acronyms JPL and CSVM [57. The data-to-MC ratios of b-tagging efficiencies obtained from these plots are used to adjust the $\mathrm{MC}$ truth predictions for use in physics measurements.

In the context of b-tagging studies [58, ATLAS defines the signed transverse impact parameter significance as $S_{d_{0}} \equiv d_{0} / \sigma_{d_{0}}$, where $\sigma_{d_{0}}$ is the uncertainty on the reconstructed transverse impact parameter $d_{0}$, with $d_{0}$ the $r-\phi$ projection of the distance of closest approach of the track to the PV. Fig. 33 shows the signed transverse impact parameter significance distribution measured in an ATLAS di-jet sample compared to a MC distribution. The overall agreement is good except in the tails of the distribution, which are more difficult to model. 


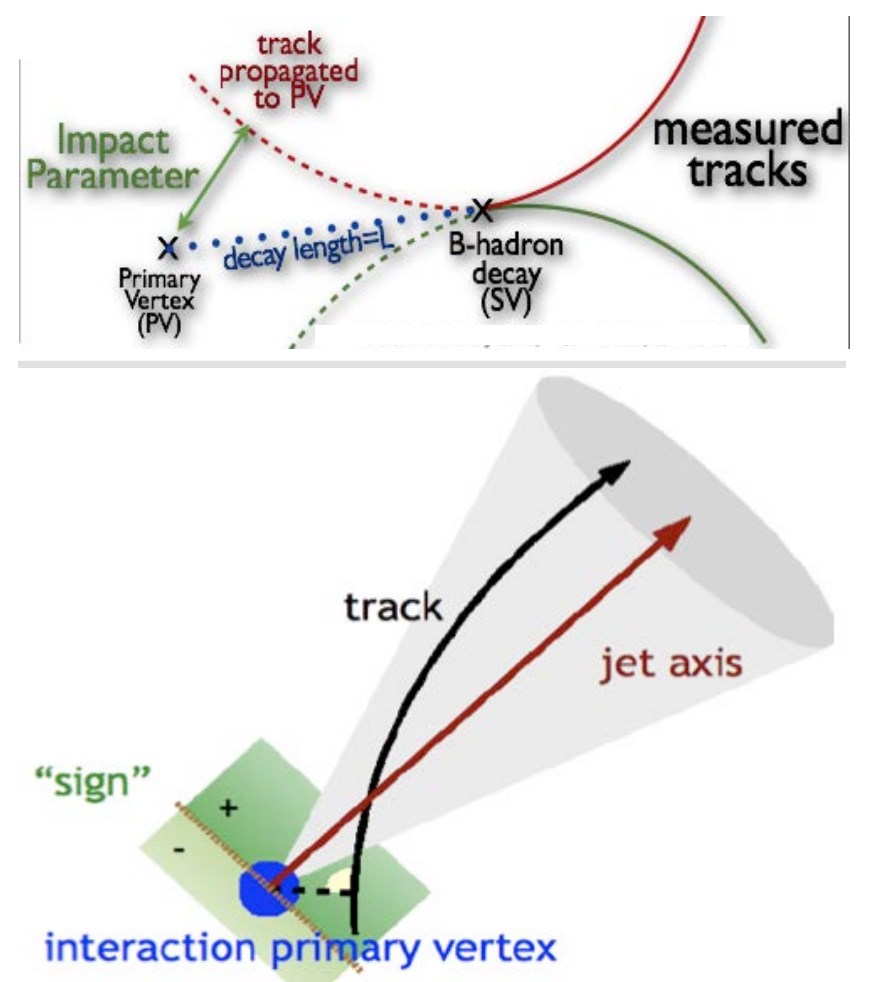

Figure 30: Top: Illustration of a $\mathrm{b}$ jet, including tracks originating from the secondary vertex (SV) and bending outwards due to the effect of the solenoidal magnetic field. The impact parameter is indicated as the distance between the primary vertex (PV) and the back-propagated tracks. Bottom: Sign convention for the impact parameter. 


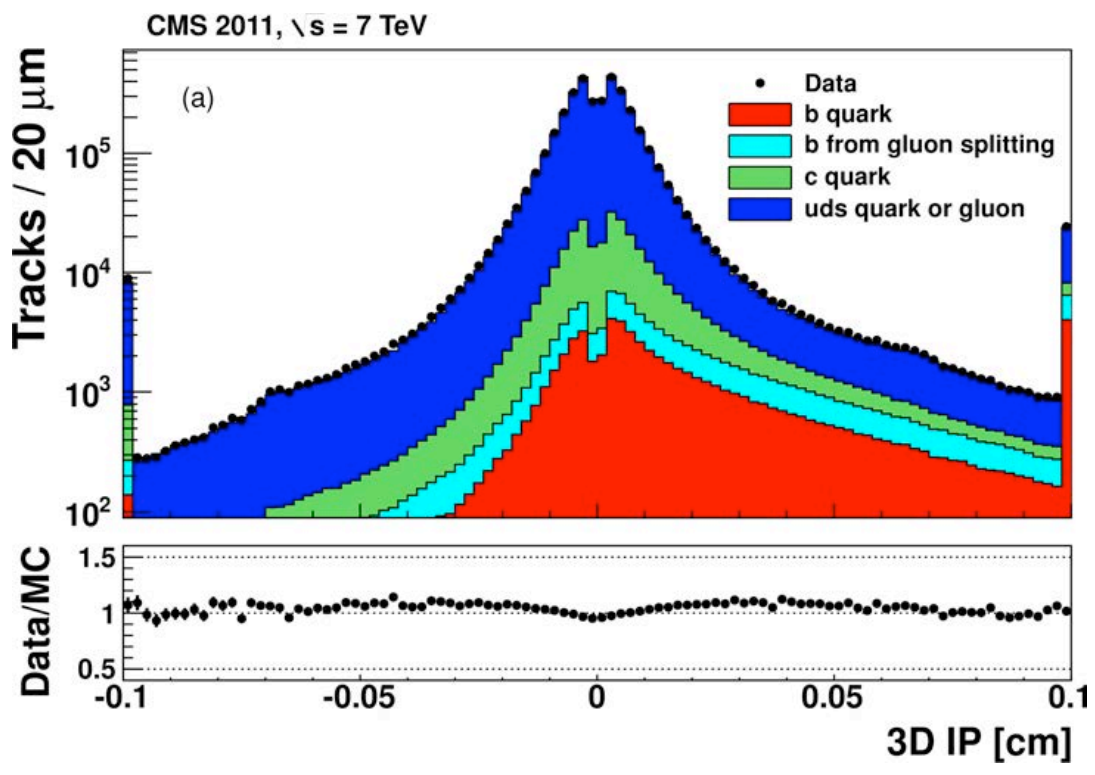

Figure 31: MC and data 3D Impact Parameter distributions for tracks in jets selected in a CMS di-jet trigger sample [57. Data is shown in full circles and the MC predictions in colored histograms. Underflow and overflow are added to the first and last bins, respectively.

The b-tagging efficiency as a function of the jet $p_{T}$ is shown in Fig 34 for a neural network tagger known by its acronym MV1. As in the case of CMS, the $\mathrm{MC}$ derivation of b-tagging efficiencies using data-driven methods is within less than $5 \%$ of the equivalent measurement in data, and the difference is accounted for in physics measurements through scale factors computed as the ratio of the values represented by the full circles over those in open squares. Differences between CMS and ATLAS efficiencies when comparing Fig. 32 with Fig. 34 are not relevant because taggers are typically tuned to different efficiency operating points depending on the fake (or mis-tag) rate tolerance for a particular physics measurement. The mis-tag probability for light-parton jets to be mis-identified as b jets is measured from data in the ATLAS and CMS experiments using "negative taggers". These inverted tagging algorithms select non-b jets using the same variables and techniques as the b-tagging algorithms. An accurate determination of the mis-tag rate is important because, since the cross section for light jets is much larger than for b jets, even a low rate of "false positives" (mis-tagged jets) affects the b-jet sample purity in a significant way. Simulating mis-tag rates is tricky because the contributing jets originate in the tails of the IP distributions, which are not trivial to model. For a mis-tag rate tolerance in the 0.01-0.03 range, CMS reports a $p_{T}$ dependence of the data-to-MC mis-tag rate scale factors of about $20 \%$ [57, while ATLAS reports factors of 2-3 for a tolerance in the 0.002-0.005 range [58]. 

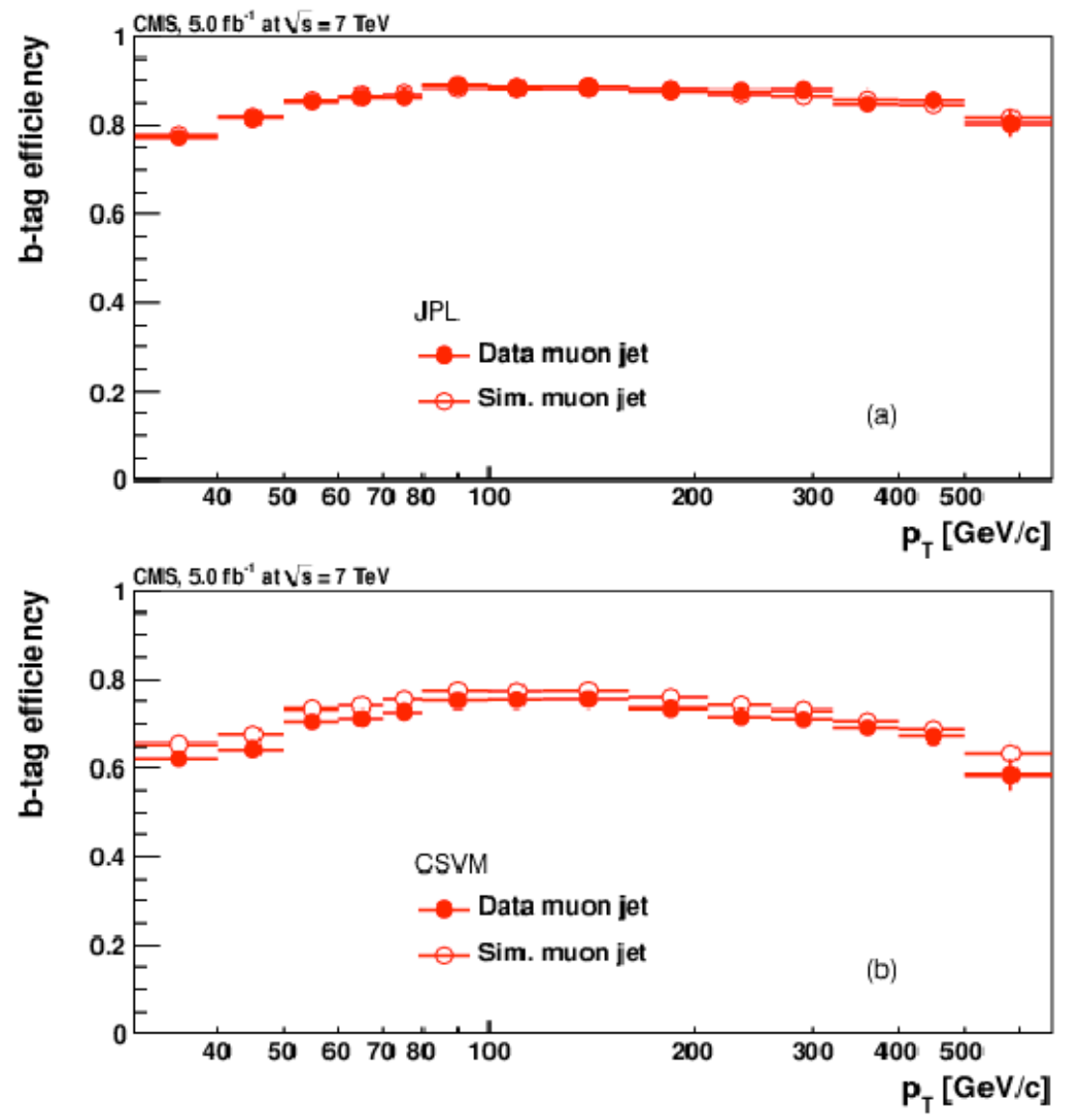

Figure 32: Efficiencies for the identification of b-jets in CMS are measured for the JPL (top) and the CSVM (bottom) b-tagging algorithms using a sample of jets with muons [57. Full and open circles correspond to data and simulation, respectively. 


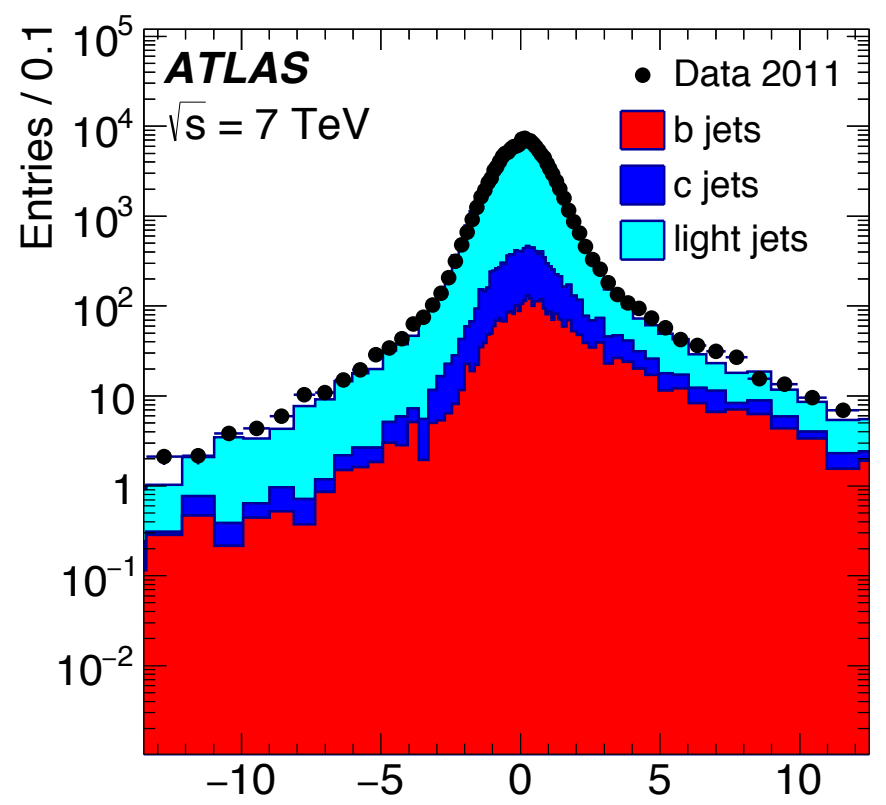

Signed transverse impact parameter

Figure 33: Signed transverse impact parameter significance distribution measured in an ATLAS di-jet sample, compared to a MC distribution [58. 


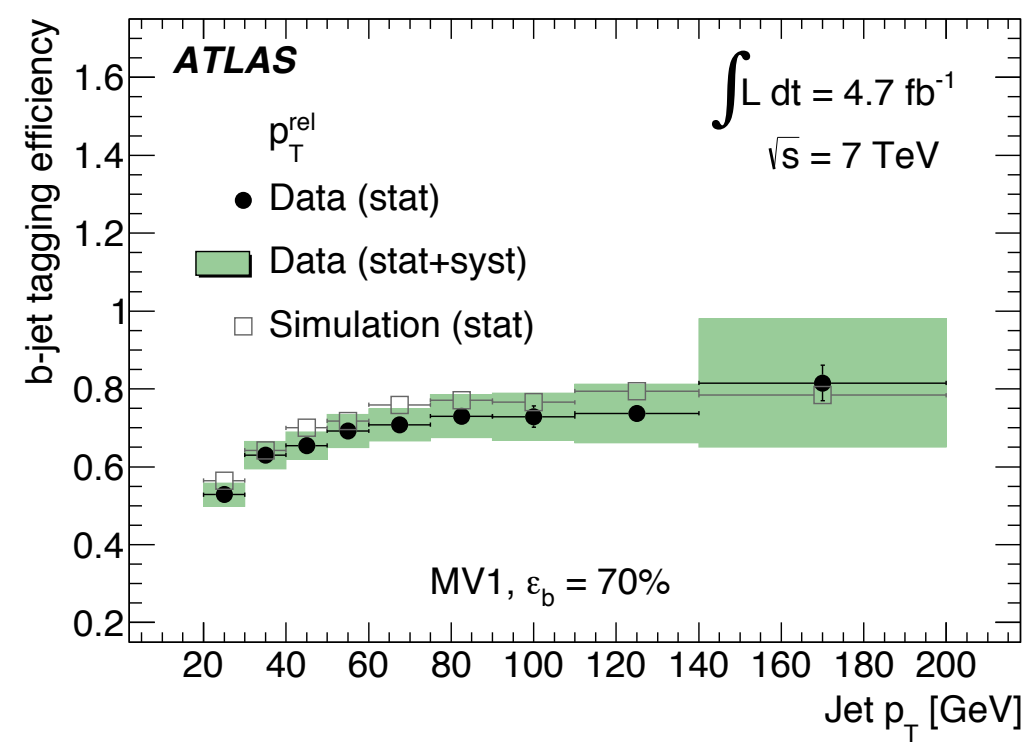

Figure 34: ATLAS's b-tagging efficiency versus jet $p_{T}$ for a the MV1 neural network tagger. Data is shown in full circles and $\mathrm{MC}$ in open squares. Error bars are statistical and the band corresponds to the systematic uncertainty in the method [58].

\subsection{2. $W, Z$ and Photon Event Distributions}

Gauge bosons such as the $W$, the $Z$ and the photon, are at the core of SM measurements and contribute backgrounds to most BSM searches. Event topologies and kinematic distributions for $W / Z / \gamma+$ jets events must therefore be modeled with high accuracy. Although physics generators are the limiting factor in the case of events with heavy flavor and many jets, the focus in this section will be on the detector modeling, clarifying when generators play a significant role.

$t \bar{t}+$ jets, $W+$ jets, $Z+$ jets backgrounds contribute at different levels to SUSY searches with jets, leptons, or photons in the final state. Although simulation is typically not used as the main tool to predict these backgrounds, MC samples are used to design and develop data-driven methods for background estimation and to perform the associated closure tests. For instance, kinematic distributions of final state particles measured in data, inspire physically motivated families of functional forms which also fit well the simulated spectra in both the control and signal regions and are ultimately used to predict the backgrounds in SR's from extrapolations of fits to data in CR's. This is a common practice in many cases where the electroweak (EWK) processes are known to be accurately modeled by physics generators. The use of MC samples to assist on the derivation of EWK backgrounds for final states with high jet multiplicity and heavy-flavor jets is more challenging, because of limitations of the physics generators rather than those of detector modeling. In other words, the standard machinery of 
the Pythia [59, 60] event generator is based on leading-level matrix elements combined with parton showers. From matrix elements calculations, the MadGraph [61] event generator produces events based on processes modeled to LO accuracy for any user-defined Lagrangian, and to the NLO accuracy for QCD corrections to SM processes. Matrix elements at the tree-level and one-looplevel can also be generated. Consequently, predictions for final states with high particle multiplicity and heavy-flavors are either inaccurate, or computationally expensive once loop-level calculations are included. Exceptionally, in the case of rare SM processes that contribute sub-dominant backgrounds, such as $t \bar{t} V, t \bar{t} H, V H$ in same-sign leptonic BSM searches, backgrounds are predicted directly from MC truth information. The cost of this approach is a large uncertainty on a small fraction of the total background, which ultimately does not affect the sensitivity of the analysis.

Fig. 35 describes the kinematics in the transverse plane of $Z+$ jets and $\gamma+$ jets collider events, which are used to illustrate the data-to-MC agreement of quantities involving gauge boson production. These events consist of either a $Z$ boson decaying to leptons or a $\gamma$ recoiling against jets that balance the transverse momentum, $\vec{q}_{T}$, of the gauge boson. The total transverse momentum of the hadronic recoil is indicated in Fig. 35 by the vector $\vec{u}_{T}$, while $u_{\perp}$ and $u_{\|}$are the components perpendicular and parallel to the gauge boson. The $E_{T}$ vector is a measure of the $p_{T}$ imbalance in the event and will be discussed in detail in Sec. 5.2 .3
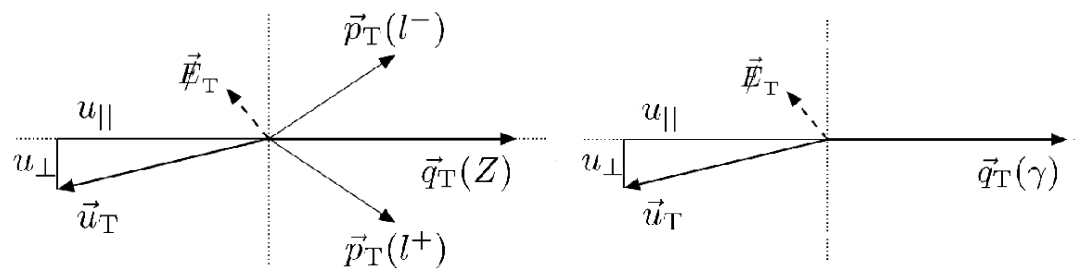

Figure 35: Kinematics of $Z+$ jets and $\gamma+$ jets events in the transverse plane. The events consist of either a $Z$ decaying to leptons or a $\gamma$ recoiling against jets that balance the transverse momentum, $\vec{q}_{T}$, of the gauge boson. The total transverse momentum of the hadronic recoil is indicated by the vector $\vec{u}_{T}$, while $u_{\perp}$ and $u_{\|}$are the components perpendicular and parallel to the gauge boson. The $E_{T}$ vector is a measure of the $p_{T}$ imbalance in the event.

Figs. 36, 37, 38 show the CMS di-lepton $\left(\mu^{+} \mu^{-}\right.$and $\left.e^{+} e^{-}\right)$invariant mass distributions for the $Z+$ jets samples, and the $\vec{q}$ distributions for the $Z+$ jets and $\gamma+$ jets samples 62 . The full circles correspond to the number of events in the data samples, while the histograms represent the MC predictions for the number of events contributed by all the physics processes with final states passing the selection criteria. The agreement between the data measurements and the MC predictions is excellent, except in the range of $q_{T}>200 \mathrm{GeV}$ for the $Z+$ jets sample, where the $\mathrm{MC}$ overestimates the data by a difference that grows linearly with $q_{T}$.

For the ATLAS experiment, Figs. 39, 40, 41, 42 depict the di-lepton invariant mass distributions in the $Z+$ jets sample and the $M_{T}=\sqrt{2 p_{T}^{l} p_{T}^{\nu}\left[1-\cos \left(\phi_{l}-\phi_{\nu}\right)\right]}$ 

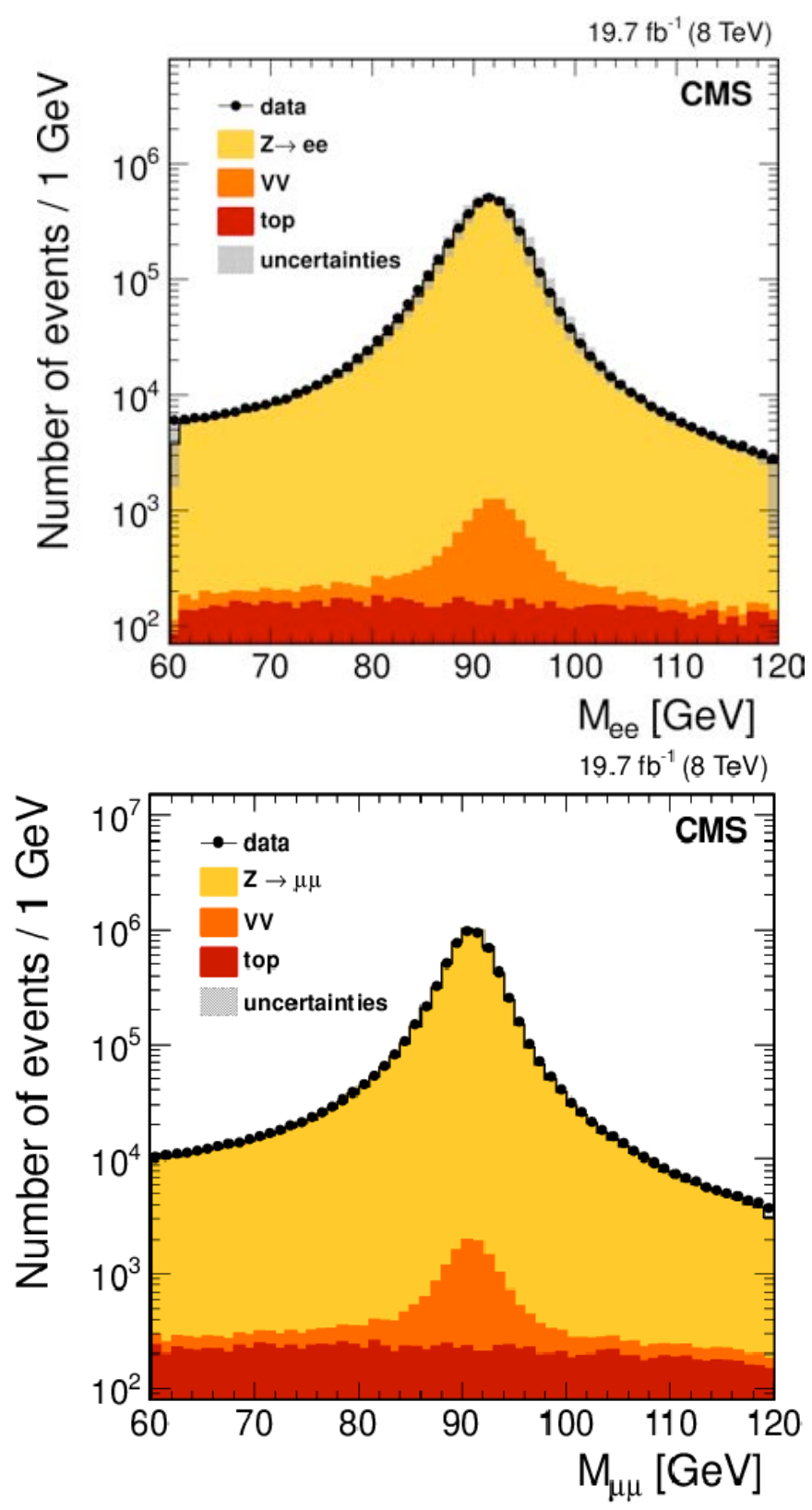

Figure 36: Di-lepton invariant mass distributions in CMS events passing the $Z \rightarrow \mu^{+} \mu^{-}$and $Z \rightarrow e^{+} e^{-}$selections 62. The full circles indicate the data measurement while the histograms are the MC predictions. The $V V$ contribution corresponds to processes with two electroweak bosons in the final state. 

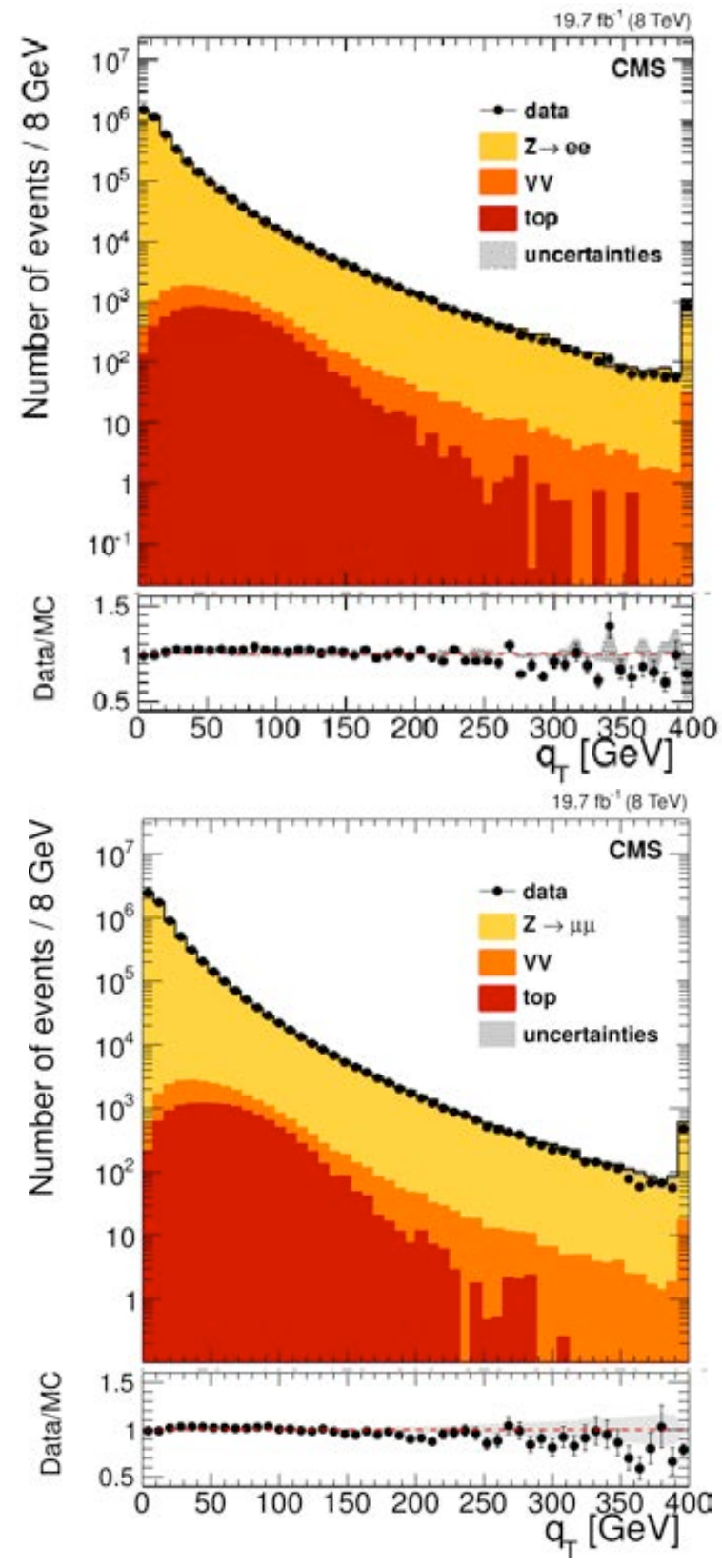

Figure 37: $Z$ transverse momentum, $q_{T}$, distributions in CMS $Z \rightarrow \mu^{+} \mu^{-}$and $Z \rightarrow e^{+} e^{-}$ events 62]. The full circles indicate the data measurement while the histograms are the MC predictions. The lower panels show the data-to-MC ratio, including the statistical uncertainties in both data and simulation, and the gray error band displays the systematic uncertainty of the measurement. 


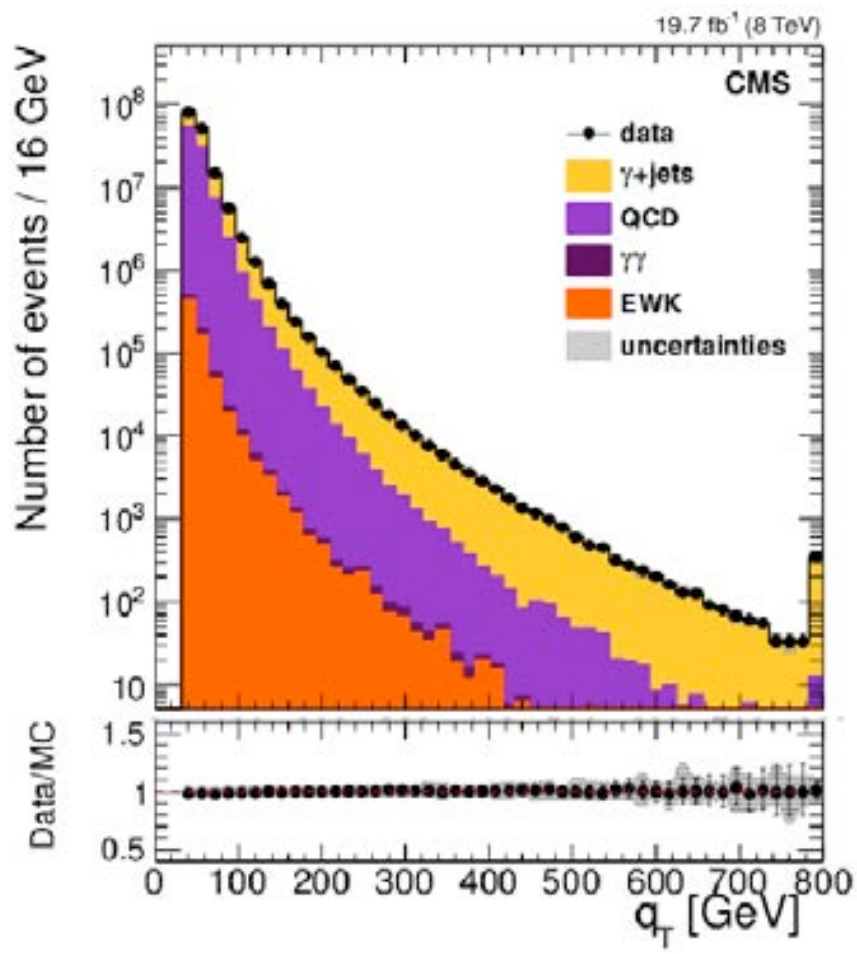

Figure 38: $\gamma$ transverse momentum, $q_{T}$, distributions in CMS direct-photon events 62. The full circles indicate the data measurement while the histograms are the MC predictions. The lower panels show the data-to-MC ratio, including the statistical uncertainties in both data and simulation, and the gray error band displays the systematic uncertainty of the measurement. 
distribution for the $W+$ jets sample for the $Z \rightarrow \mu^{+} \mu^{-} / e^{+} e^{-}$and $W \rightarrow \mu \nu / e \nu$ decay channels [63. The full circles correspond to the data, while the histograms represent the $\mathrm{MC}$ predictions for all the physics processes with final states and kinematics passing the selection criteria. The MC prediction agrees with the data within the systematic uncertainties in all cases, an impressive result given that the uncertainties are $<10 \%$ for most distributions in the domain ranges with good statistics.

\subsubsection{Missing Transverse Energy Distributions}

The missing transverse energy, denoted $E_{T}$ or $E_{T}^{\text {miss }}$, is defined as the negative vector sum of the transverse components of the energy of all particles in the event. Although this term is physically incorrect because energy is a scalar quantity, it is widely used in high-energy particle physics because calorimeters measure energy (not momentum) and, in most events of interest in current collider experiments, particle masses are negligible with respect to their total energy. In this limit of negligible mass, energy equals momentum and missing transverse momentum may be approximated by $E_{T}$ as defined above. Modeling $E_{T}$ is one of the most challenging simulation tasks because this event level quantity depends on accurate simulation of all types of particles, including hadronic showers from jets, as well as unclustered energy not assigned to any particle in the event. Challenging as it is, accurate modeling of $E_{T}$ in simulation is of paramount importance to the quality of BSM searches for SUSY and Extra Dimensions (ED), as well as in collider-based searches for dark matter. Simulation of $E_{T}$ also played a crucial role in the discovery and characterization of the Higgs boson, particularly in the $H \rightarrow \tau \tau$ final state, and channels with a $W \rightarrow l \nu$ or a $Z \rightarrow \nu \nu$.

The $E_{T}$ distibution in $W / Z+$ jets events is a key ingredient of many SM measurements and BSM searches. Events with a $W$ or a $Z$ and many jets have intrinsic $E_{T}$ when the $W$ decays to $e \nu / \mu \nu$ or the $Z$ decays to $\nu \nu$, and spurious $E_{T}$ coming from jet energy resolution effects. As a result, these processes contribute significant background to most searches for signals with weakly interacting particles because the latter have a large $E_{T}$ signature, and the $W / Z+$ jets events may have large fake $E_{T}$. The level of understanding of $W / Z+$ jets $E_{T}$ distributions and the quality of their simulation modeling in CMS and ATLAS is illustrated in Figs. 43, 44, 45 for $Z \rightarrow \mu^{+} \mu^{-} / e^{+} e^{-}$and $W \rightarrow \mu \nu / e \nu$ decays 62, 63. The data-to-MC ratios show that the nominal differences are less than $20 \%$ for CMS $Z$ +jets $E_{T}$ distributions, and less than $10 \%$ for ATLAS $Z / W+$ jets distributions. In both experiments, systematic uncertainties grow above $50 \%$ in different ranges of the $E_{T}$ domain. In CMS, uncertainties are largest in the $50-90 \mathrm{GeV}$ range where the contribution of hadronic shower mis-measurement dominates.

All-jet events resulting from strong production of highly collimated beams of particles are among the most difficult to simulate and so is their associated $E_{T}$. To model the high-energy tail of the $E_{T}$ distribution of these multi-jet events, the simulation needs to include a high degree of detail in the development and fluctuations of particle showers in the detector, as well as modeling of rare oc- 

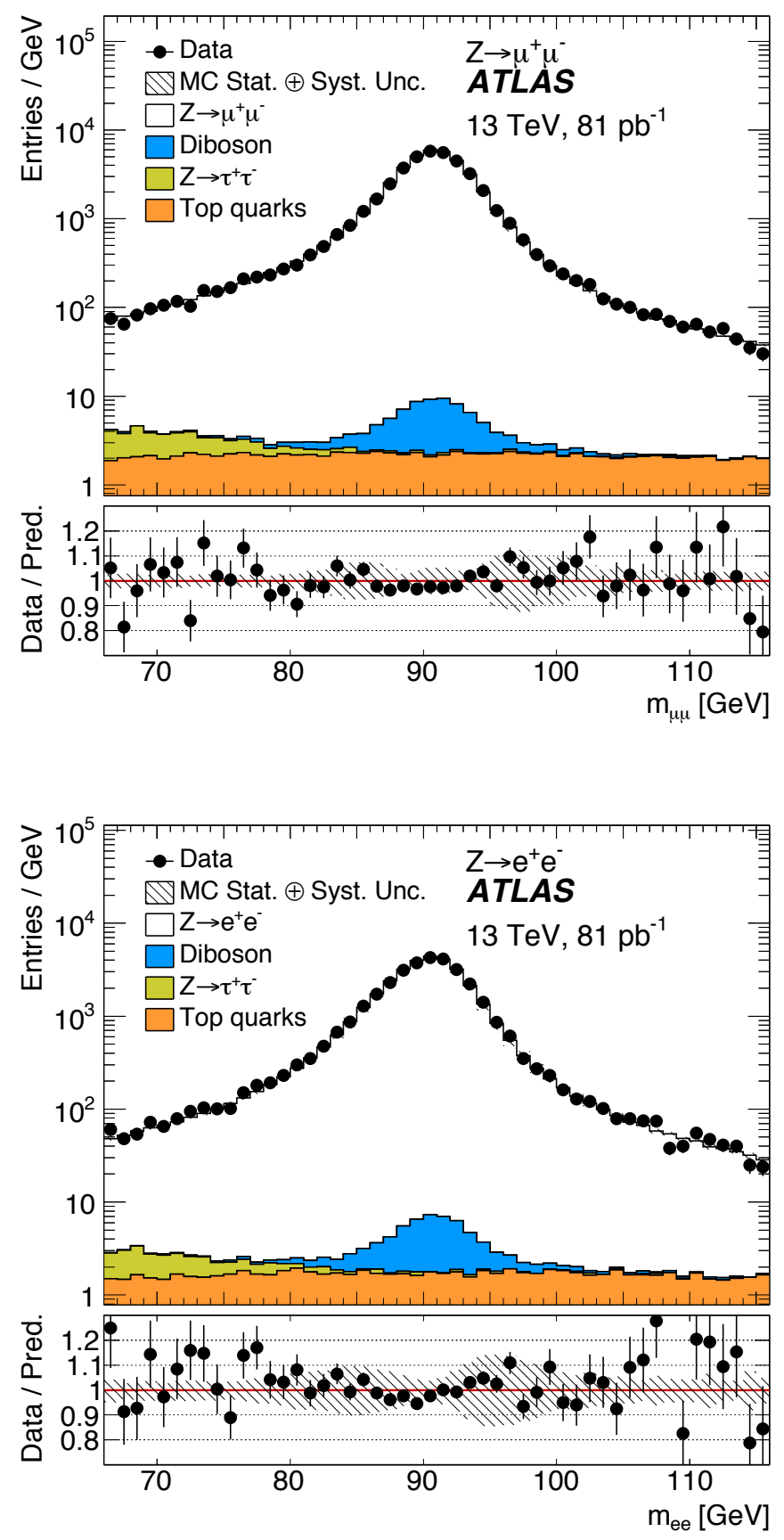

Figure 39: Di-lepton invariant mass distributions in an ATLAS $Z+$ jets sample for the $\mu^{+} \mu^{-}$ and $e^{+} e^{-}$decay channels 63 . The full circles correspond to the data, while the histograms represent the MC predictions for all the physics processes with final states and kinematics passing the selection criteria. Error bars are statistical and the shaded band shows the systematic uncertainty in the data measurement. 

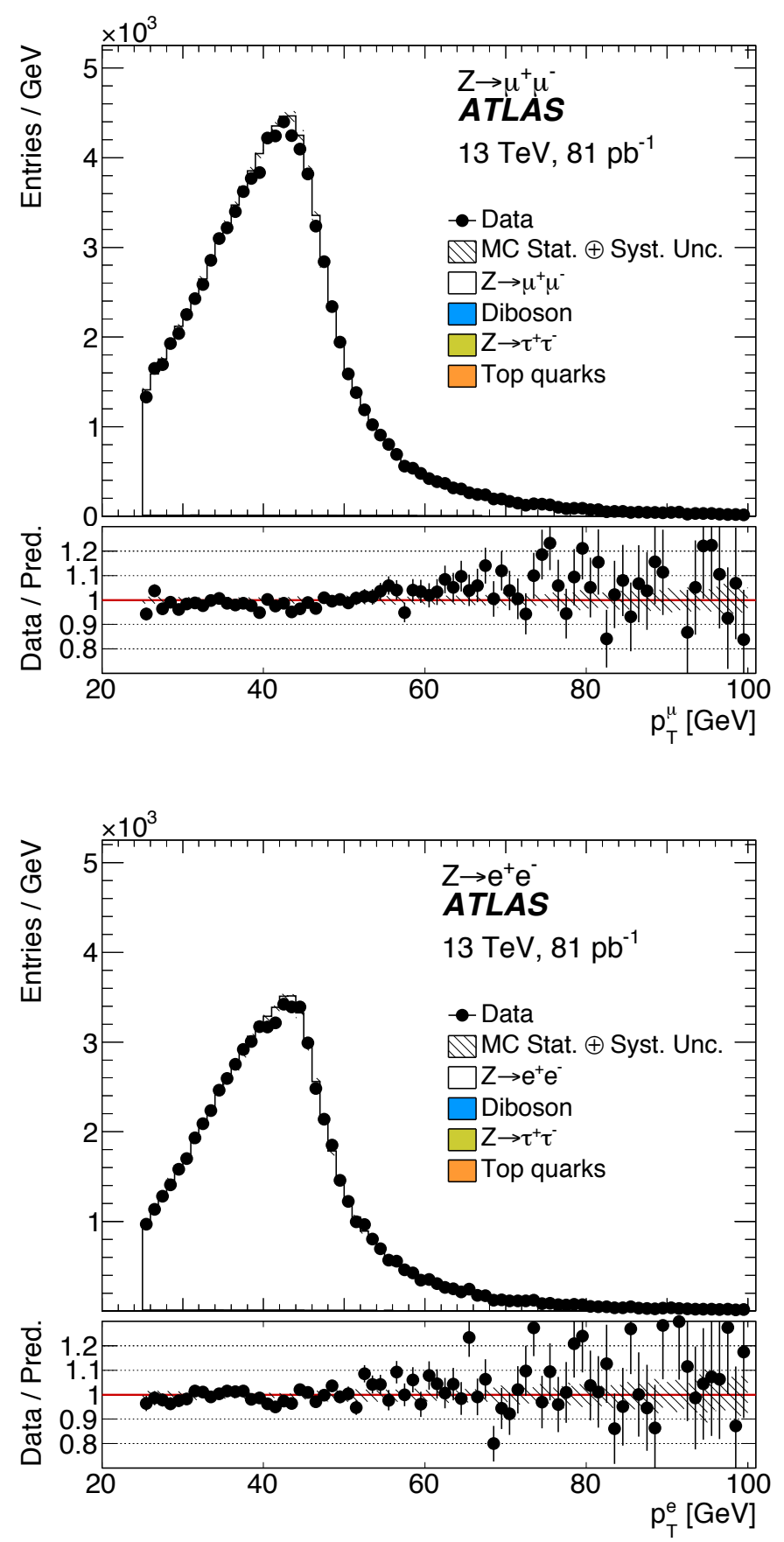

Figure 40: Muon and electron transverse momentum distributions in an ATLAS $Z+$ jets sample for the $\mu^{+} \mu^{-}$and $e^{+} e^{-}$decay channels 63 . The full circles correspond to the data, while the histograms represent the MC predictions for all the physics processes with final states and kinematics passing the selection criteria. Error bars are statistical and the shaded band shows the systematic uncertainty in the data measurement. 

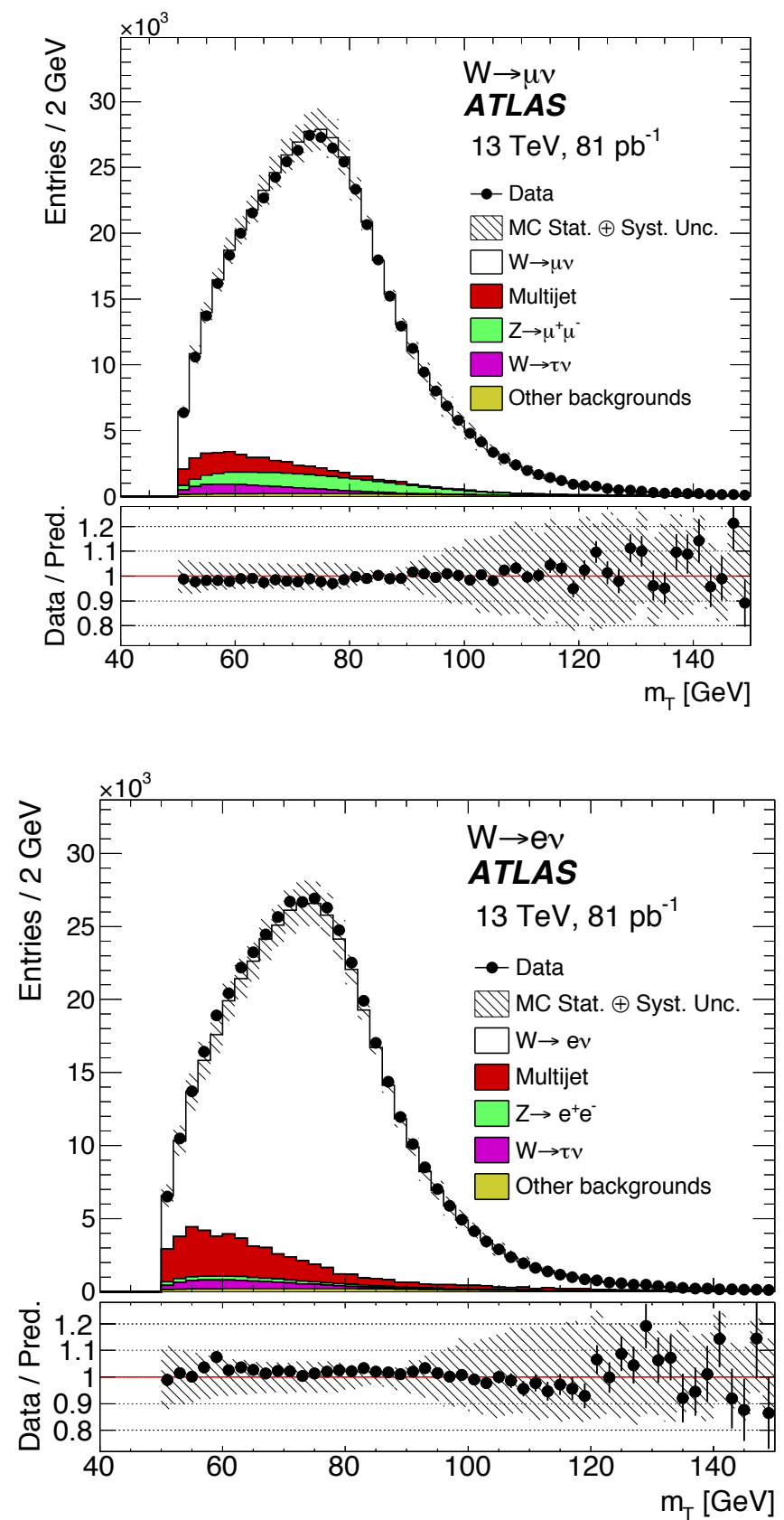

Figure 41: Transverse mass distribution, $M_{T}=\sqrt{2 p_{T}^{l} p_{T}^{\nu}\left[1-\cos \left(\phi_{l}-\phi_{\nu}\right)\right]}$, in an ATLAS $W+$ jets sample for the $\mu \nu$ and $e \nu$ decay channels 63. The full circles correspond to the data, while the histograms represent the MC predictions for all the physics processes with final states and kinematics passing the selection criteria. Error bars are statistical and the shaded band shows the systematic uncertainty in the data measurement. 

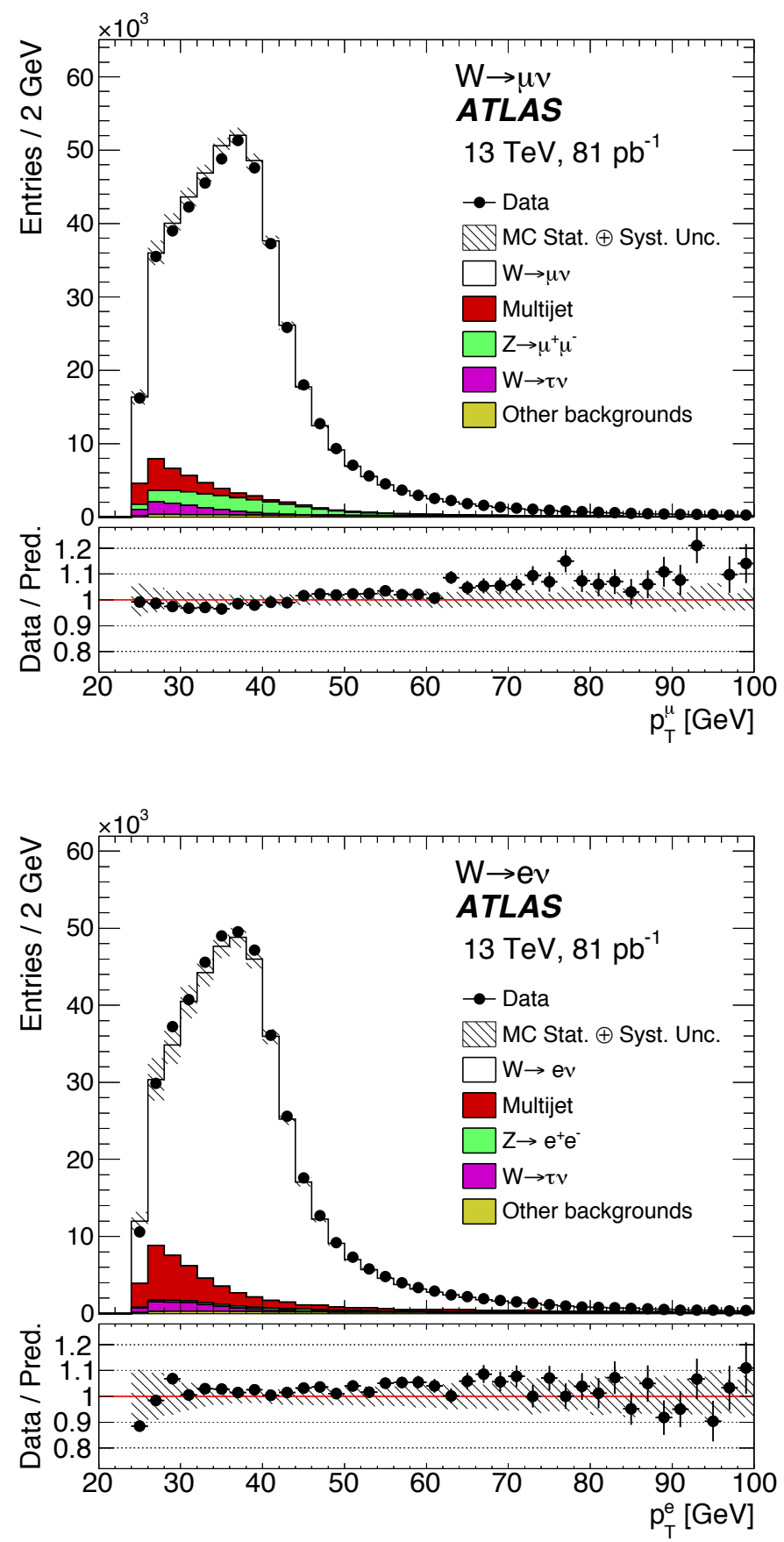

Figure 42: Transverse momentum distributions in an ATLAS sample of $W+$ jets events for the $\mu \nu$ and $e \nu$ decay channels 63. The full circles correspond to the data, while the histograms represent the MC predictions for all the physics processes with final states and kinematics passing the selection criteria. Error bars are statistical and the shaded band shows the systematic uncertainty in the data measurement. 

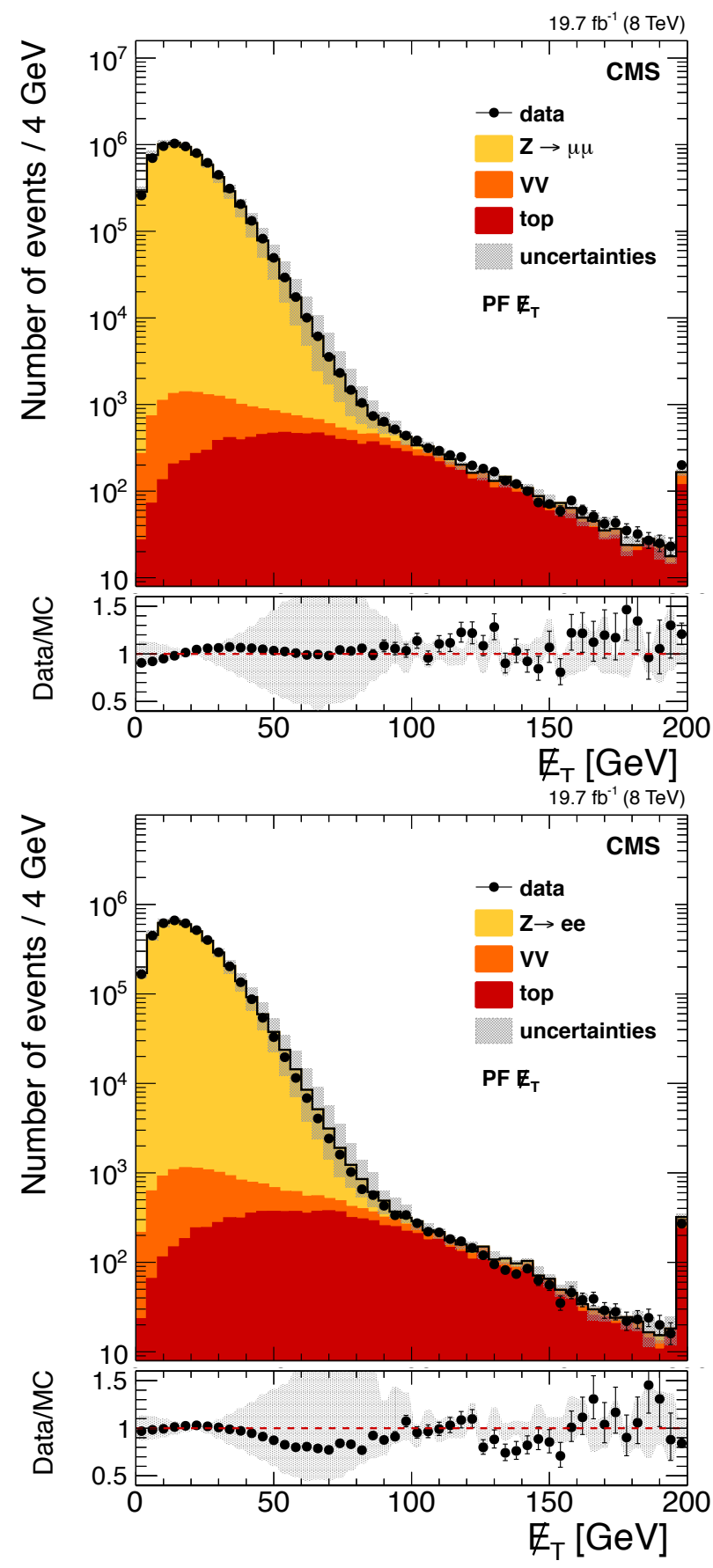

Figure 43: Missing transverse energy distribution in a CMS $Z+$ jets sample for the $\mu \mu$ and $e^{+} e^{-}$decay channels 62 . The full circles correspond to the data, while the histograms represent the MC predictions for all the physics processes with final states and kinematics passing the selection criteria. Error bars are statistical and the shaded band shows the systematic uncertainty in the data measurement. 

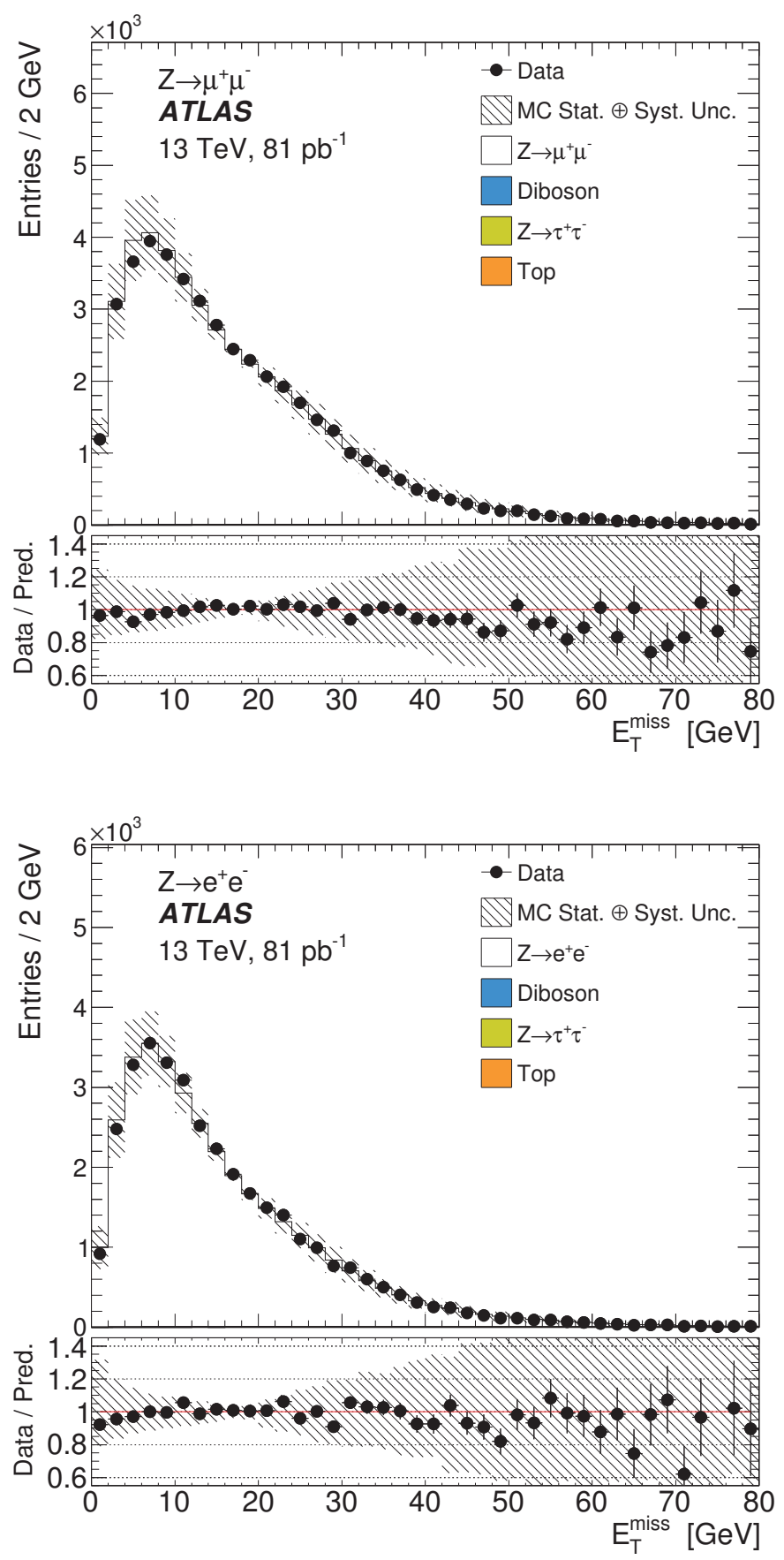

Figure 44: Missing transverse energy distributions in an ATLAS $Z+$ jets sample for the $\mu \mu$ and $e^{+} e^{-}$decay channels 63 . The full circles correspond to the data, while the histograms represent the MC predictions for all the physics processes with final states and kinematics passing the selection criteria. Error bars are statistical and the band shows the systematic uncertainty in the data measurement. 

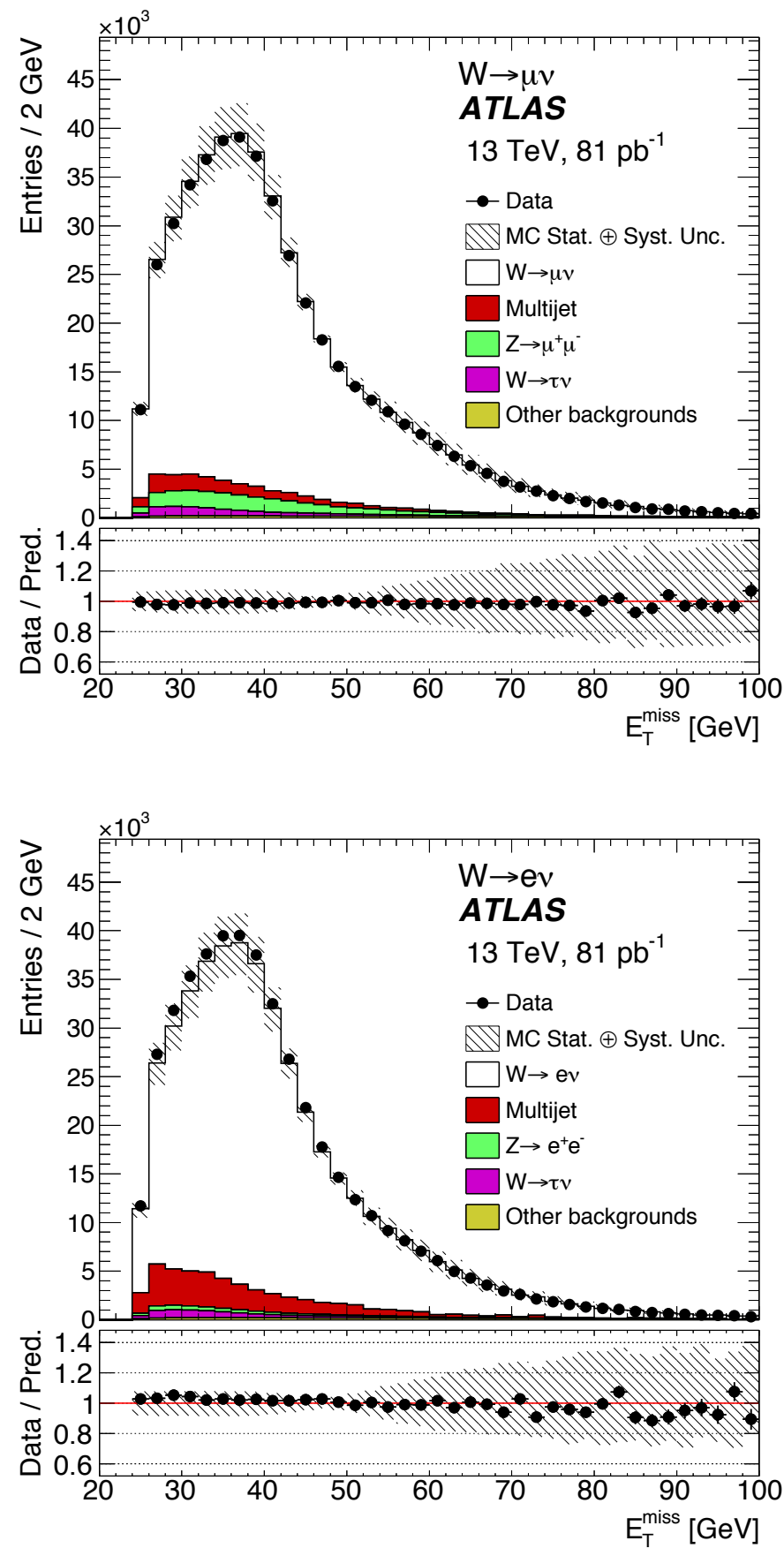

Figure 45: Missing transverse energy distributions in an ATLAS $W+$ jets sample for the $\mu \nu$ and $e \nu$ decay channels 63. The full circles correspond to the data, while the histograms represent the MC predictions for all the physics processes with final states and kinematics passing the selection criteria. Error bars are statistical and the band shows the systematic uncertainty in the data measurement. 
currences of detector signal processing malfunctions. Consequently, MC-truth predictions of $E_{T}$ distributions are typically not reliable, to the level required in modern collider experiments, to make accurate estimates of the background contributions of these QCD events to measurements of other SM processes and potential BSM signals. The $E_{T}$ simulation challenge in multi-jet events originates in the fact that jets are composed of many particles with energies ranging from a few $\mathrm{GeV}$ to hundreds of $\mathrm{GeV}$, which shower into hundreds of more particles as they traverse the detector material. Different models for electromagnetic and nuclear interactions and a careful handling of the model-to-model transitions are required to simulate these showers, depending on the particle type, energy, and material involved. Small changes in the modeling of energy fluctuations translate into large differences in the transverse momentum imbalance observed in multi-jet events, which ranges from mild to severe and result in small to very large fake missing transverse momentum.

More examples of backgrounds to SM and BSM measurements with $\mathscr{E}_{T}$ are presented below. Backgrounds are irreducible when they come from a physics process that results in a final state indistinguishable from the one for the signal. Reducible backgrounds are those that are distinguishable from the signal due to distinctive physics properties. Irreducible QCD background to $t \bar{t}$ production occurs for the final state where both top quarks decay hadronically to two b jets plus light jets. In this case, the multi-jet event observed in the detector is indistinguishable from an event with the same light-jet and b-jet multiplicity which originates in the strong production and subsequent hadronization and fragmentation of light quarks, b quarks, and gluons. Instead, when a $t \bar{t}$ pair decays semi-leptonically, QCD background arises from the measurement process, when jets in the tails of the response distribution cause fake $E_{T}$ that mimics the $W \rightarrow l \nu$ process in top decays. A SUSY search with all jets in the final state, where events with large $E_{T}$ are selected, is another example of instrumental QCD background. Events in the tails of the jet response distribution, observed in Fig. 12, make large contributions to SUSY SR's due to their large production cross sections. The source and rates of these rare events with extremely large $E_{T}$ are very difficult to identify, evaluate, and eventually simulate.

Data-to-MC comparisons of $\mathscr{E}_{T}$ distributions are presented next to illustrate the level of simulation accuracy achieved in modern experiments despite the many challenges described above. Fig. 46 shows the $\mathscr{E}_{T}$ distribution for CMS di-jet events before and after applying the software algorithms to remove events with spurious $\mathscr{E}_{T}[62$. Excellent agreement is observed in the $>500 \mathrm{GeV}$ range, even in the tail of the distribution. Agreement deteriorates below $500 \mathrm{GeV}$ as the contribution of QCD events in the sample increases and eventually becomes dominant. In spite of the excellent agreement observed in the high $E_{T}$ range, data-driven methods are preferred over MC predictions of multi-jet backgrounds with high $E_{T}$, particularly in searches with a SR in the tail of the $\mathscr{E}_{T}$ distribution, and simulation-based closure tests are utilized to demonstrate accuracy. The reason is that it is basically impossible to demonstrate that all sources of spurious events in this region of low statistics have been identified, understood, and modeled in the $\mathrm{MC}$ with the correct rates of occurrence. One example of 
these rare occurrencies is when a high-energy particle hits directly a photo-diode in the detector readout circuit.

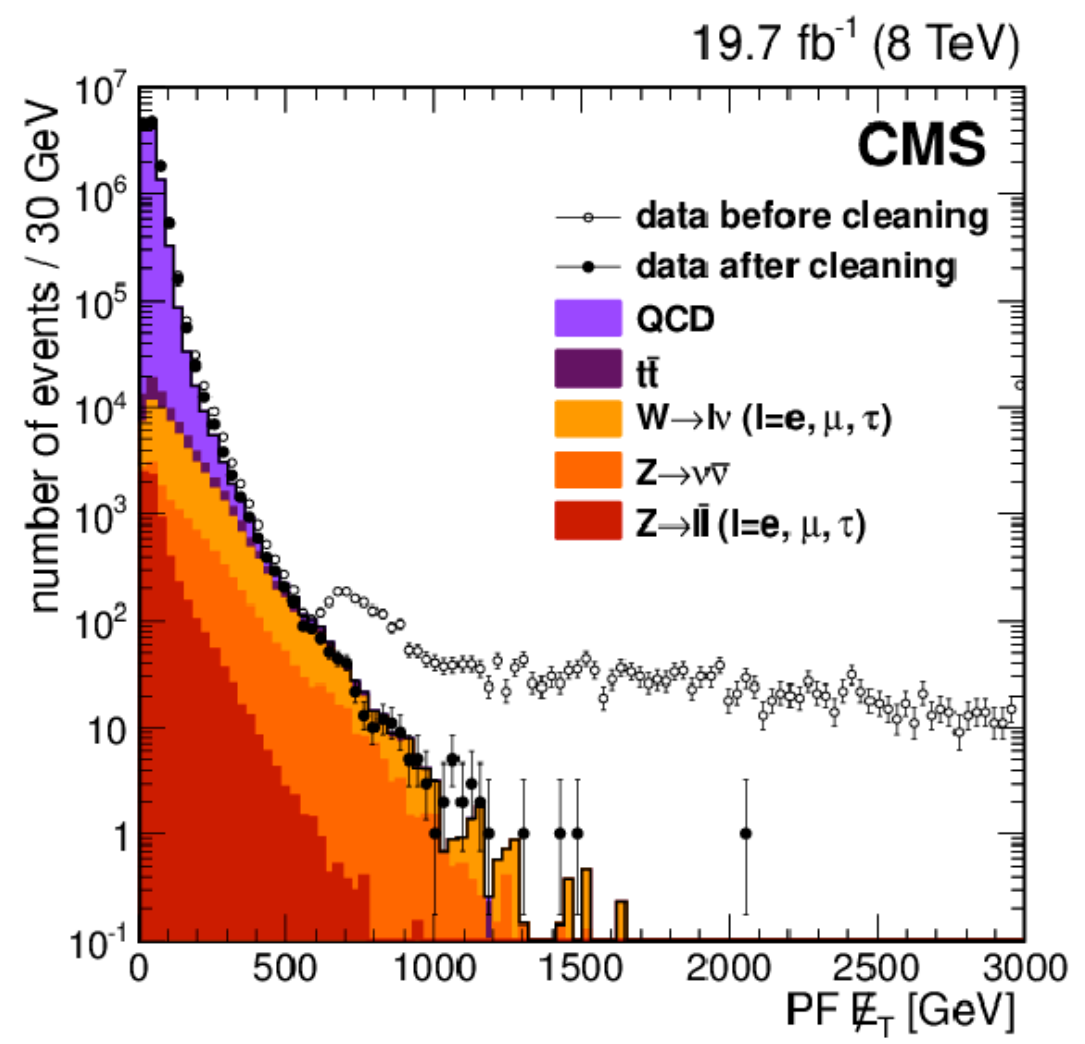

Figure 46: Particle Flow $\mathscr{E}_{T}$ distributions for CMS di-jet events with (full circles) and without (open circles) the fake events removal algorithms applied. The SM contributions to the simulated $E_{T}^{\prime}$ distribution are shown in histograms 62.

Fig. 47 shows the resolution of the CMS particle flow $E_{T}$ projections along the $x$ and $y$ axes as a function of the scalar sum of the transverse energy, $\Sigma E_{T}$, of all the reconstructed particles in the event, except for the $\gamma$ in the $\gamma+$ jets sample or the leptons from the decay of the $Z$-boson candidate in the $Z+$ jets sample 62 . $E_{T}$ resolutions in $Z+$ jets events for the $e^{+} e^{-}$and $\mu^{+} \mu^{-}$ decay channels are described in the simulation within a $10 \%$ accuracy, well within the statistical and systematic uncertainties of the measurements. A good modeling of the energy resolutions of measured particles and event quantities such as $E_{T}$ is important because small data-to-MC discrepancies would cause a different amount of distribution "smearing" and therefore bin-to-bin migration of events. A poor modeling of the resolution smearing effect would render the MC of limited use in physics analysis. Migration effects may be large and are challenging to simulate, particularly in the case of the jet $p_{T}$ spectrum, 
which reflects the rapidly falling $p_{T}$ dependence of the QCD cross sections for jet production. In this case, the event $E_{T}$ distribution would be significantly affected in a detector with poor energy resolution because low $p_{T}$ jets produced with large cross sections would populate the high $E_{T}$ tail, reducing the purity in the highest $E_{T}$ bins of the distribution.

For the ATLAS experiment, Fig. 48 shows a plot of the RMS obtained from the combined distribution of the $x$ and $y$ components of $E_{T}$ versus the scalar sum of the $E_{T}$ of the physics objects in a $Z+$ jets sample [64. The data is presented in full markers and the MC in open markers for different alternative $\mathscr{E}_{T}$ calculations. The $E_{T}$ is reconstructed as the negative vector sum of calibrated physics objects ( $e$ 's, $\gamma^{\prime}$ 's, $\tau$ 's, jets, $\mu$ 's) and a soft term that comprises all the detector signals not matched to physics objects. CST, TST, STVF, EJAF, and Track refers to different algorithms to reconstruct and calibrate the soft term, based on different combinations of tracker and calorimeter information, and pile-up subtraction techniques. Over the whole $\Sigma E_{T}$ domain, the agreement of MC with data is always better than $5 \%$.

\subsection{Simulation and Jet Cross Sections}

The example of jet cross sections and QCD jet measurements in general is particularly useful to illustrate the impact of simulation in data measurements because of its dependence on a single dominant source of systematic uncertainty, the jet energy correction, which in turn relies to a large extent on how well the hadronic response and energy resolutions are modeled in the simulation. In particular, the energy response to low-energy hadrons $(E=1-10 \mathrm{GeV})$ is the most difficult to model and affects even high-energy jets given that the energy of the jet constituents grows slowly, approximately as the square root of the jet energy.

Figs. 50, 49, 51, 52 do not show the latest and most precise measurements of the ATLAS [65], CMS [66], CDF [67, and D0 [68] inclusive jet cross sections. Instead, they show the results in the first publications of each experiment based on the full 2010 Run 1 ATLAS and CMS samples, the full 1992-1993 Run 1a CDF sample and the full 1993-1995 Run 1b D0 sample. The goal here is to analyze the impact of simulation in the publication process timeline and the precision of the result. There is an important difference between the comparisons for jet measurements and the rest of the data-to-MC comparisons in this article. While the latter are comparisons between detector-level measured quantities and predicted quantities based on events generated and passed through detector simulation software, the former are comparisons between measured quantities corrected to the particle level and NLO-QCD parton level theoretical predictions which, sometimes, contain non-perturbative hadronization corrections. In the inclusive jet measurements, all detector effects such as jet energy response and resolution smearing have been removed, in average, as part of the analysis procedure. Therefore, theoretical predictions do not need to be passed through detector modeling software in order to be on the same footing for comparison with data. While comparisons in previous sections give information about the quality of the event generators and detector simulation software 

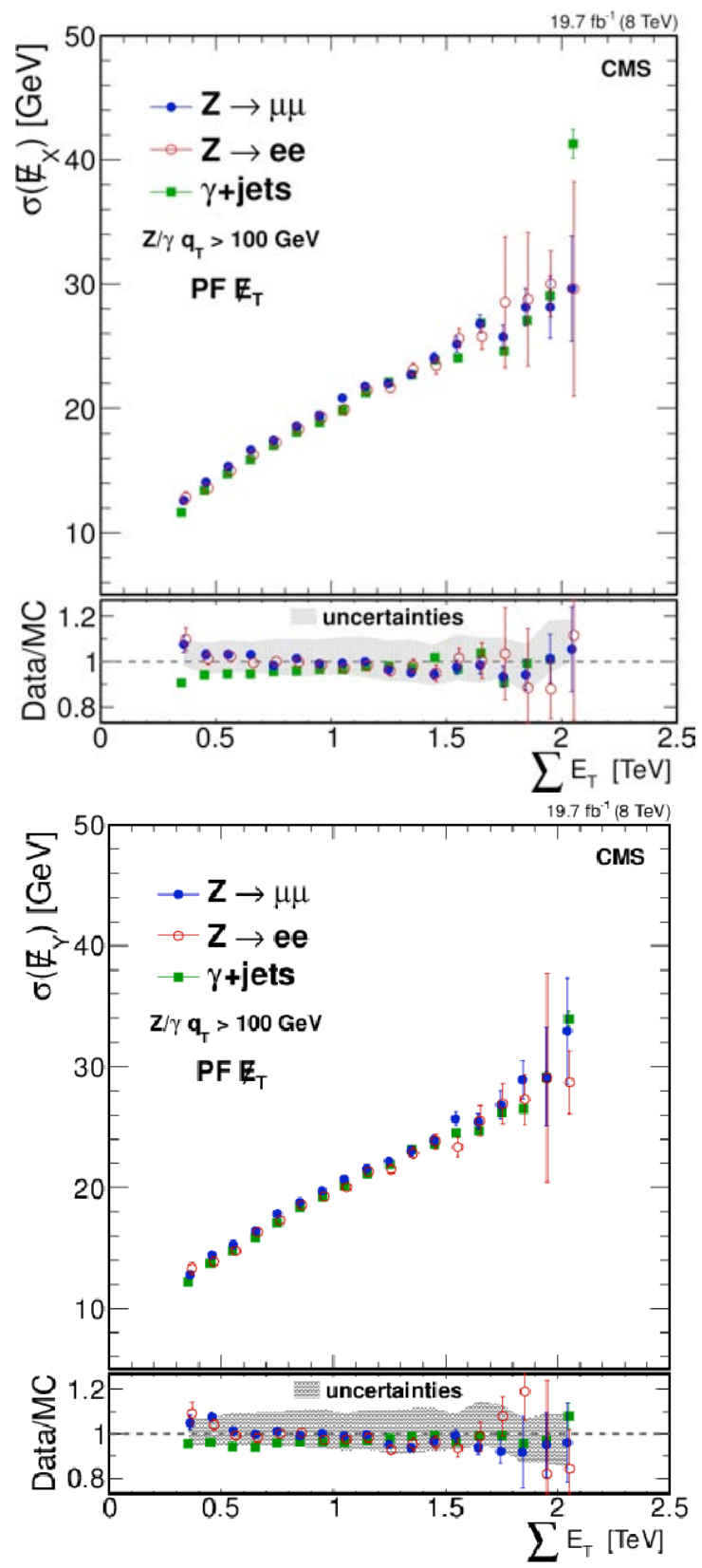

Figure 47: Resolutions for the CMS $E_{T}^{\prime}$ projections along the $x$ and $y$ axes as a function of the scalar sum of the transverse energy, $\Sigma E_{T}$, of all the reconstructed particles, except for the $\gamma$ in the $\gamma+$ jets sample or the leptons from the decay of the $Z$ boson candidate in the $Z+$ jets sample 62. The error bars are statistical and the shaded band represents the systematic uncertainties. 


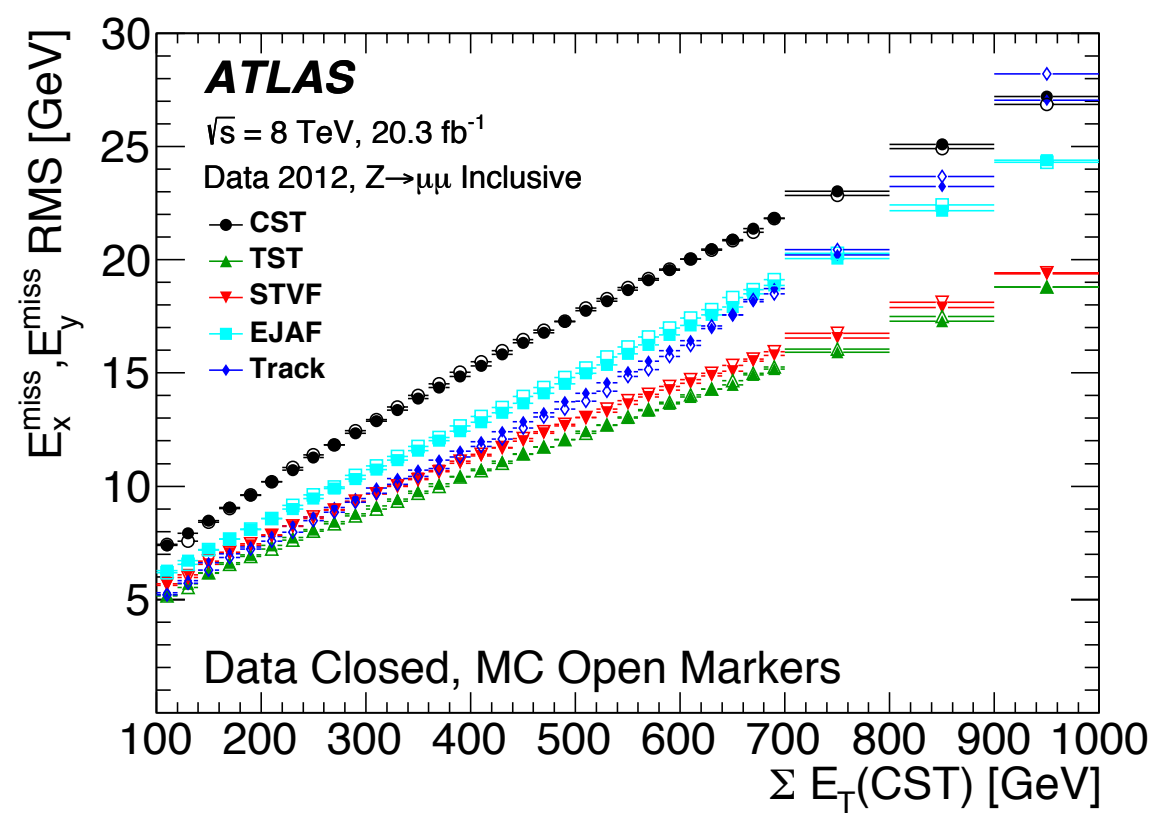

Figure 48: RMS of the ATLAS distribution obtained from the combination of the $x$ and $y$ components of $E_{T}$ as a function of the scalar sum of the transverse energy, $\Sigma E_{T}$, of all reconstructed physics objects in an ATLAS $Z \rightarrow \mu^{+} \mu^{-}+$jets sample 64]. 
tools, the jet cross section comparisons evaluate the accuracy of the QCD theoretical predictions, which depend on the order of the calculation, the choice of factorization and renormalization parameters, and the parton distribution functions (PDFs). The aspect of the jet cross section measurements to highlight in this section is the relationship between the size of the systematic uncertainty (measurement precision), the role of simulation, and the publication timeline (publication turnaround). The capabilities of the detectors as well as the quality of the FullSim and ParSim tools, utilized to either design the data-driven jet correction derivation methods or directly extract the corrections after thorough tuning and validation, dominate the accuracy of the measurements and the publication timeline.

Fig. 49 shows the CMS inclusive jet cross section measurements based on the full $34 \mathrm{pb}^{-1}$ data collected in 2010. The data taking period started in March 2010 and the results were published in June 2011, only seven months after the end of the run. The jet cross sections shown in Fig. 50 are based on the full 2010 data set and were published by the ATLAS experiment in April 2012. ATLAS also published an intermediate result [69], based on half the data-set, in October 2010, before the end of the run. The CMS measurement extends up to rapidities of $|y|=3$ with uncertainties in the range of $10-20 \%$ in the most central region and $15-30 \%$ in the most forward bin. ATLAS measured the jet cross sections up to rapidities of $|y|=4.4$ with uncertainties similar to those in the CMS measurement for the central bin and in the $12-40 \%$ range for the most forward bin. Rapidity is defined as $y=\frac{1}{2} \ln \left[\left(E+p_{z}\right) /\left(E-p_{z}\right)\right]$, where $E$ is the jet energy and $p_{z}$ its momentum component along the beam axis.

The CDF experiment published the $19.5 \mathrm{pb}^{-1}$ Run 1a data-set in January 1996, almost five years after the start of the run at the Tevatron. The measurement, shown in Fig. 51, covers only the central pseudorapidity region, $0.1<|\eta|<0.7$, with uncertainties in the $20-35 \%$ range. D0's first inclusive jet cross section measurement, shown in Fig. 52, was published in 1999, eight years after the start of Run 1. It was based on the full $92 \mathrm{pb}^{-1}$ Run $1 \mathrm{~b}$ data-set, restricted to the $|\eta|<0.5$ range, and reported uncertainties in the $10-30 \%$ range. A few years later, in 2001, D0 extended the Run 1 measurement to the forward pseudorapidity region, up to $|\eta|=3$ [70]. Both experiments published the Run 2 inclusive jets cross sections, CDF in $|\eta|<2.1$ (2008) [71], and D0 in a slightly larger, $|\eta|<2.4$, region (2011) 72 . The reason for the CDF delay in extending the $\eta$ coverage is that the experiment initially tuned the ParSim only for the central calorimeter. The End Plug Calorimeter was not incorporated until a GFLASH based approach was undertaken in 2002-2003. Jet energy calibration in forward regions relies on di-jet balance techniques, which are significantly affected by resolution biases and can be understood in detail only with large and accurate MC samples. In the case of D0, a ParSim approach was not viable due to the absence of a solenoidal magnetic field in the tracker and scarce test beam data. The in situ calibration approach based on data-driven methods applied to collider data had to be developed from scratch and without the aid of large and accurate FullSim samples for studies and closure tests. Consequently, D0 could not deliver a result with competitive systematic uncertainties until 1996, 

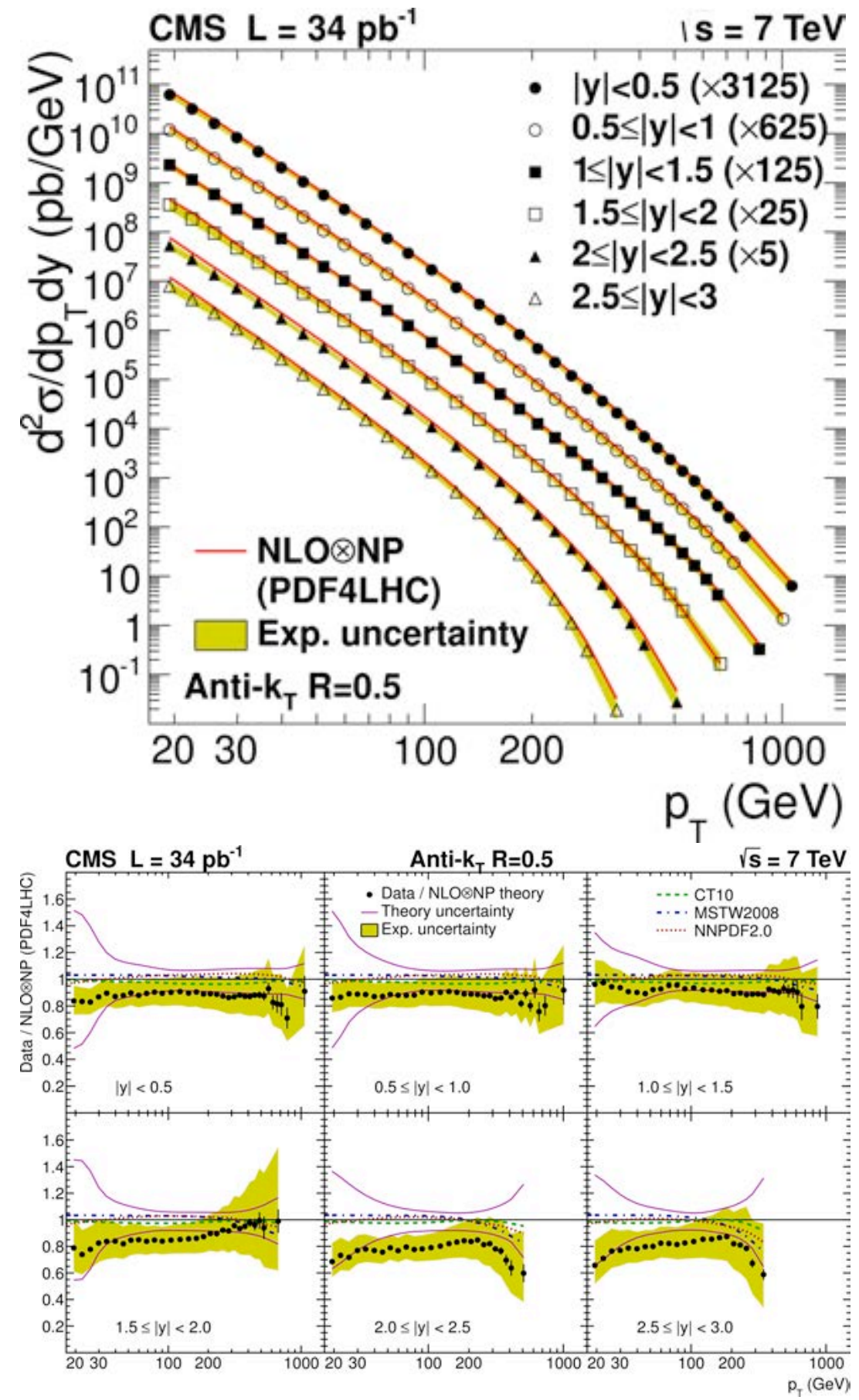

Figure 49: First inclusive jet cross sections published by the CMS experiment, based on the full 2010 (Run 1) sample 66. 

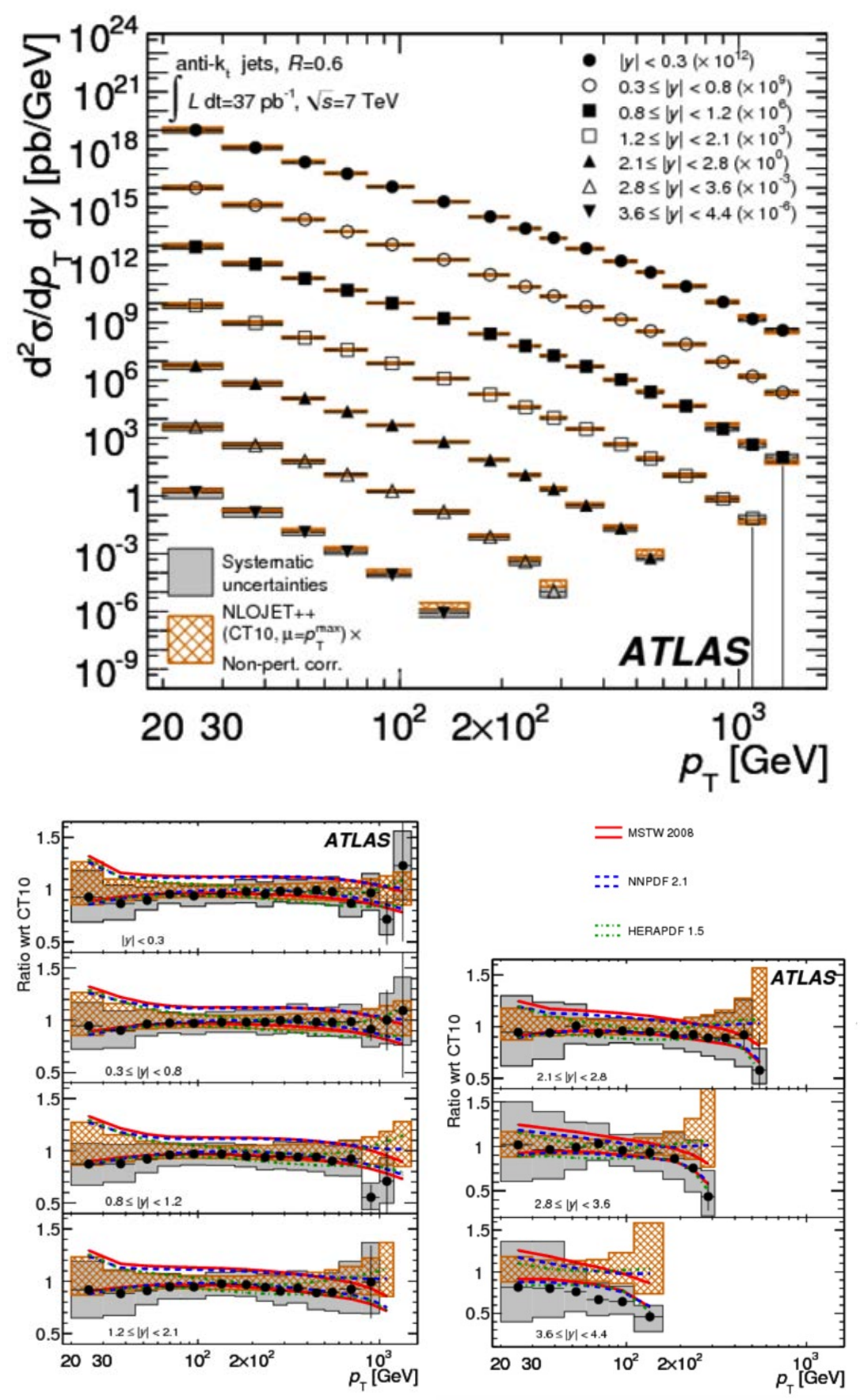

Figure 50: First inclusive jet cross sections published by the ATLAS experiment, based on the full 2010 (Run 1) sample 65. 
while CDF published jet cross sections with large uncertainties in 1989 (Run 0 ) [73 and 1992. The latter was an intermediate Run 1 result based on early data [74.

The Tevatron inclusive jet cross section story is one of limited test beam programs, complex tuning of parametrized simulations, and a lengthy process of developing data-driven techniques with little aid from full simulation. The LHC experiments benefited from new generation detectors with excellent capabilities, mature data-driven techniques and expertise, simulation of unprecedented quality, and a computing infrastructure with the capacity to generate not hundreds of thousands but billions of MC events. While it took months to CMS and ATLAS to publish jet cross section results with uncertainties on the order of $10-40 \%$ (10-20\% in the most central region), it took years to D0 and CDF to achieve a level of precision that was a factor of two inferior.

\section{Simulation and Publication Turnaround}

The process of publication of physics measurements from start-up to paper submission has accelerated significantly in modern particle physics experiments. Although many technological and human factors account for this trend, including the fact that the LHC experiments have thousands of members and the Tevatron experiments hundreds at their peak, simulation has played a significant role. Figs. 53, 54 show the number of publications per year between 1998 (1992) and 2014 (2016) for the CDF 75] (D0 [76]) experiment at the Tevatron, and the integrated number of publications as a function of time for the CMS experiment at LHC 77. For the Tevatron experiments, Run 1a started in June of 1992 and finished by the end of the spring of 1993. Unlike D0, CDF had a Run 0 in 1988-1990. As illustrated in Fig. 53, while the first D0 physics paper was published in early 1994, the publications distribution for Run 1a peaked in 1995, three years after the start of the run. Run 1b started in 1994, the publications distribution peaked in 1998 and began to slow down in 2001. The absence of a Run 0 explains D0's delay with respect to CDF in submitting the first Run 1a publications, since the experiment had to commission the detector, optimize the software algorithms and develop analysis techniques using the early data.

In the absence of fast enough simulation, GEANT3 was available but was computationally costly given the speed of the machines at the time, the process of developing data-driven techniques from the scratch was a challenging and lengthy process for both Tevatron experiments. As discussed in Sec. 2, CDF used Run 0 data to measure the single-track energy response from minimum bias and track triggers to tune a fast MC. This parametrized simulation approach was preferred during Run 0 and Run 1 over the full GEANT3-based option because the latter was prohibitively slow. In Run 1, D0 did not have a solenoid magnet wrapped around the tracker to measure the momentum of single-charged-particles and tune the simulation. Consequently, the experiment relied purely on in situ measurements of calibration factors and efficiencies using data-driven methods applied to collider events. The process of developing these methods and, eventually, improving and tuning their simulation software 

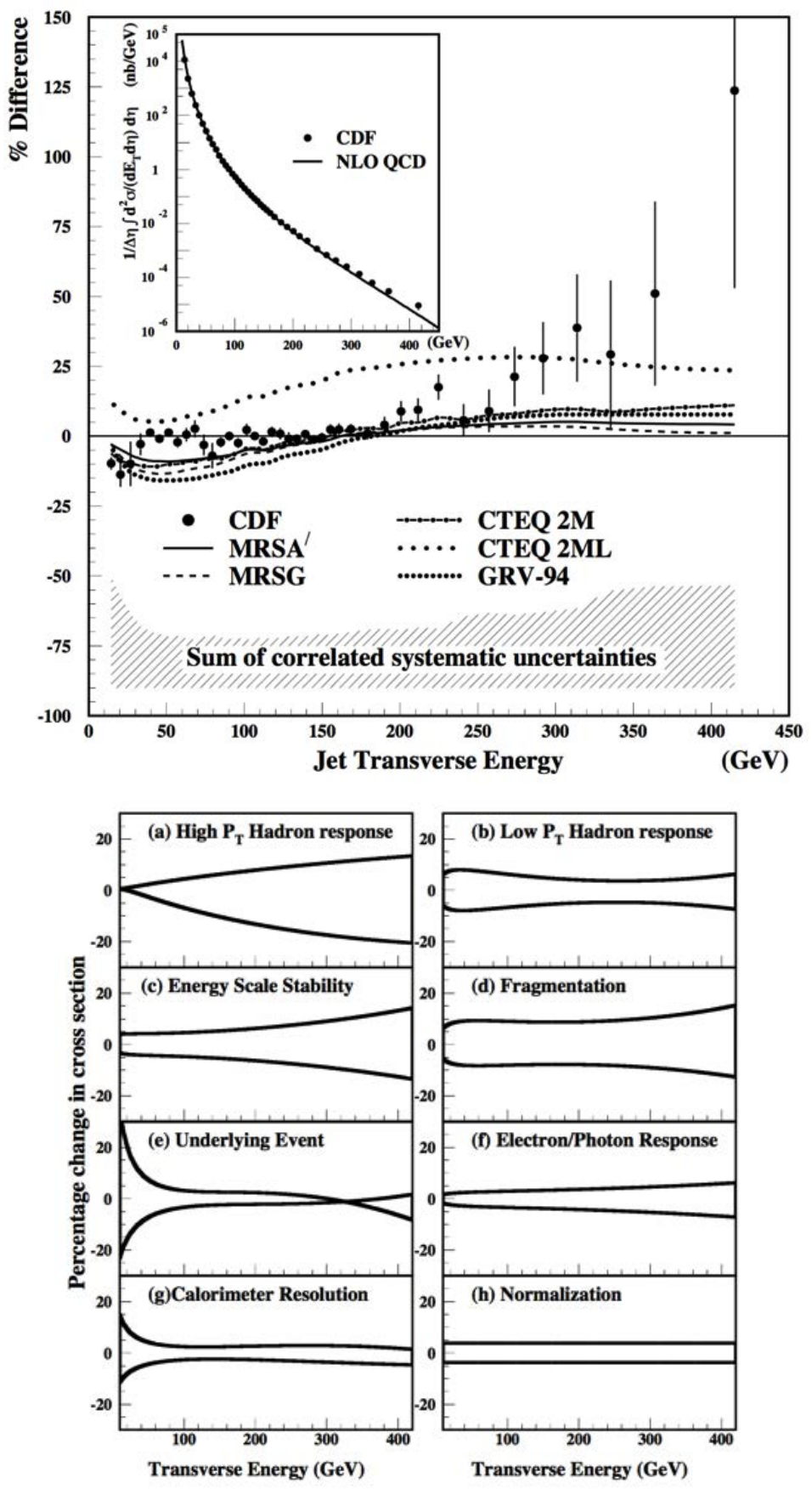

Figure 51: First inclusive jet cross sections published by the CDF experiment, based on the full 1992-1993 (Run 1a) sample 67. 

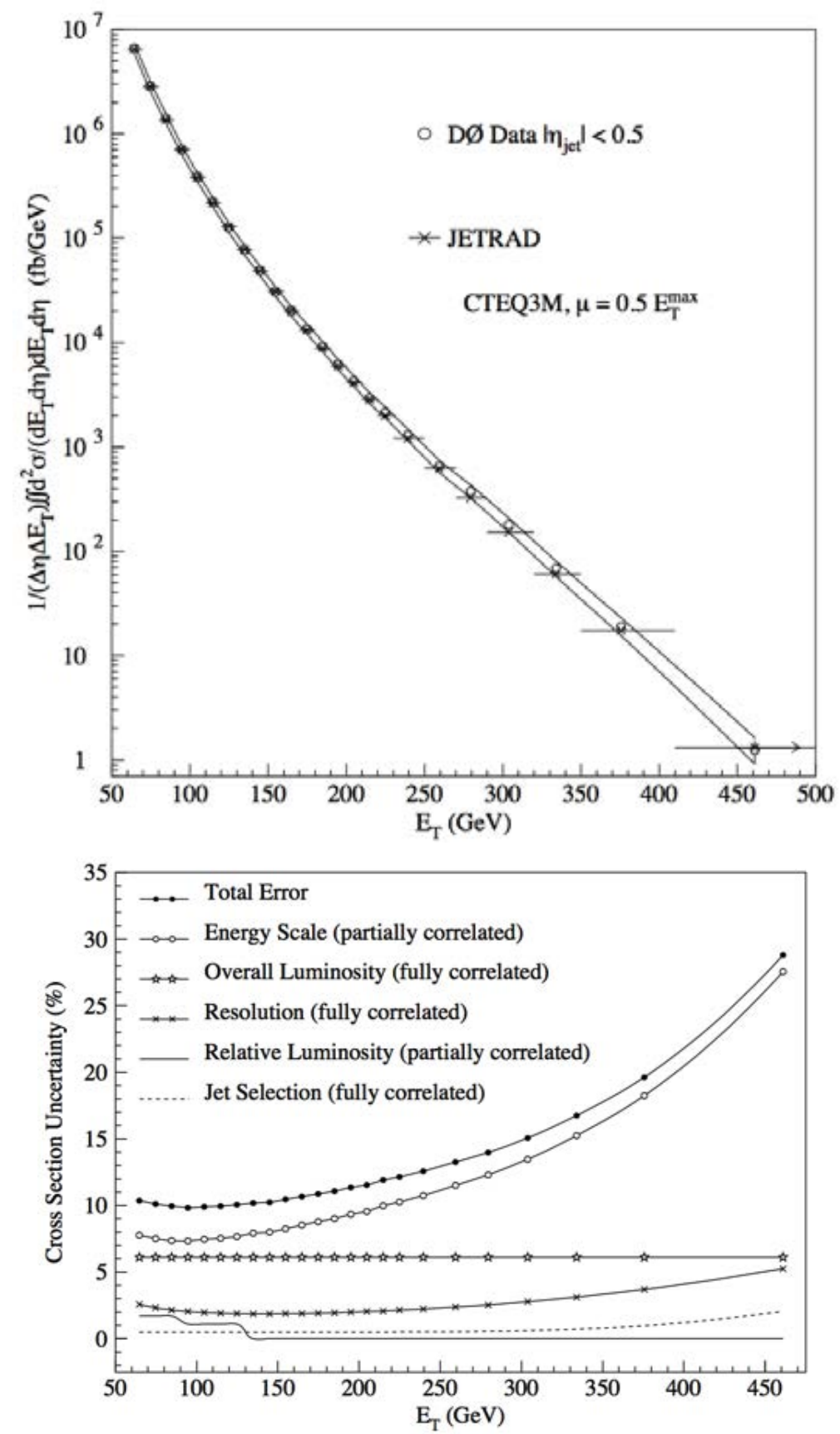

Figure 52: First inclusive jet cross sections published by the D0 experiment, based on the full 1993-1995 (Run 1b) sample 68. 
was very lengthy for the experiments because they had to rely on small MC samples, of the order of a few ten to a few hundred thousand events, to develop the techniques and demonstrate their correctness via closure tests. Even with the aid of GEANT3-based simulation software, accuracy was often sacrificed for speed by introducing approximations to the sub-detector shapes, material, or particle shower modeling. The resulting MC samples were only partially useful to develop data-driven techniques, investigate their associated biases, and establish closure. At the LHC, hundreds of millions of fully simulated events were generated using Geant4-based applications even before the start of the first run. Reconstruction algorithms and data-driven methods to derive efficiencies and calibration factors were developed using these MC samples, and performed, basically, as in design specifications on real collider data at start-up. MC truth predictions of calibration curves and physics observables were in such good agreement with data, that they could be used "out-of-the-box" almost immediately after start-up, requiring only small corrections and even smaller uncertainties derived from comparisons with results from data-driven methods applied on real collider data.

The highly accurate simulation software of the LHC experiments, fast computing and precise data-driven techniques, which leveraged the Tevatron experience, contributed to a large extent to the much faster publication turnaround at the LHC.

\section{Economic Impact and Cost of Simulation in HEP Experiments}

Simulation, including physics generation, interaction with matter (Geant4 or ParSim), readout modeling, reconstruction and analysis takes a large fraction of the computing resources consumed in HEP experiments. The estimate of this number for the CMS experiment presented in this article has a large uncertainty and it varies significantly year-to-year. Since the software commissioning period in preparation for Run 1, the Geant4 part of the CMS simulation software chain has taken the largest fraction of the CPU time, while the physics generation contribution has been small, except in the case of the generation of BSM signal samples in a large model parameter space. Readout modeling takes a relatively small fraction and reconstruction of the same order as the Geant4 module.

From start-up in 2009 through May 2016, CMS simulation as defined in the first sentence took approximately $85 \%$ of the total CPU time utilized by CMS, while the Geant4 module took about $40 \%$. (This information was obtained from the CMS Dashboard, which is a computing information monitoring source available to CMS members.) ATLAS's Geant4 module takes approximately seven times more CPU time than CMS's due to the more complex geometry and other factors. In CMS, the rest of the CPU cycles were primarily used to reconstruct and analyze real collider data. The assumption for the $85 \%$ figure is that the analysis of simulated data consumes $75 \%$ of the CPU time spent in analysis, including both simulated and real data, and excludes the generation of signal samples for BSM searches. The reason why the analysis of simulated data takes a larger fraction of the total analysis CPU time than the analysis of 

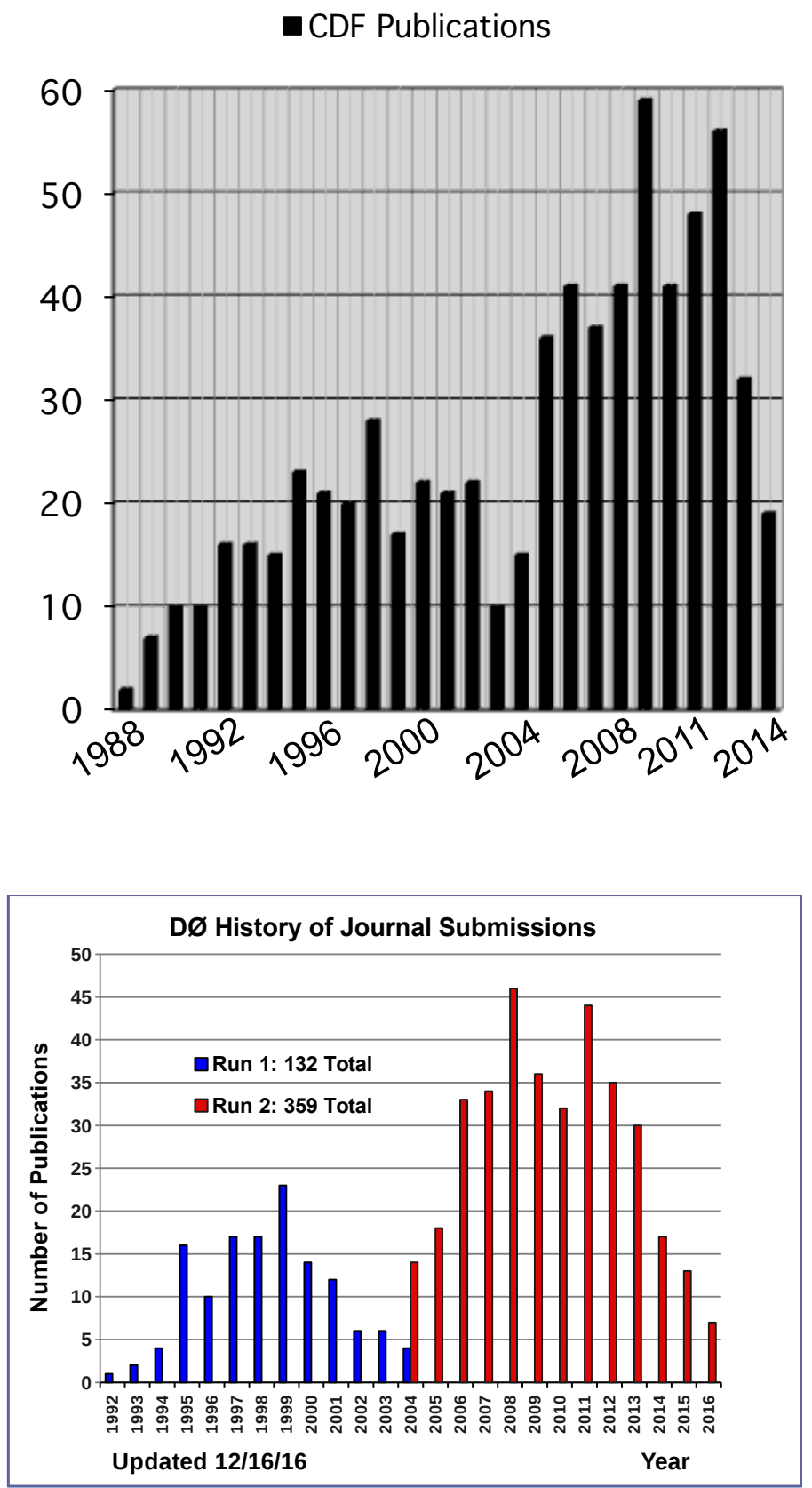

Figure 53: Number of publications per year for the CDF [75] and D0 [76] experiments at the Tevatron. 
567 collider data papers submitted as of 2016-12-16

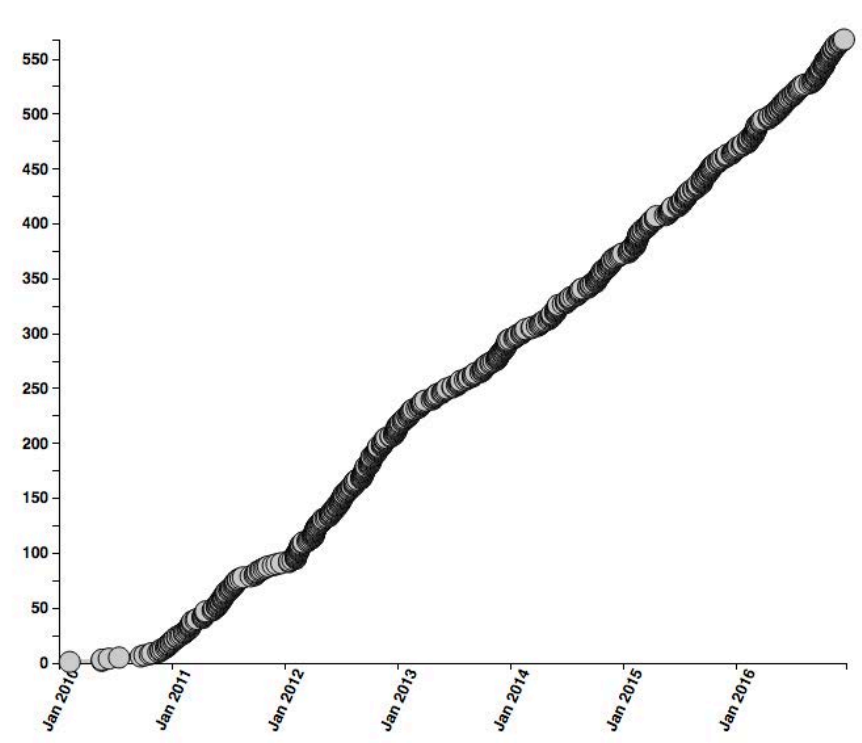

Figure 54: Integrated number of publications as a function of time for the CMS 77 experiment at the LHC.

real collider data is that the design and optimization of the measurements, as well as the development and validation of data-driven methods, are all based on MC samples.

In more detail, CMS spent on simulation 540 thousand core months during 2012 (860 thousand core months in the May 2015-May 2016 period), corresponding to more than $45,000(70,000)$ CPU cores at full capacity that cost on the order of 5 (8) million US dollars. (This information was obtained from the CMS Dashboard and from private communication with Oliver Gutsche.) These numbers account only for purchasing cost though, and a more realistic estimation may be based on a value of 0.9 US dollar cents per core hour, which is what Fermilab spends on physical hardware including life-cycle, operation and maintenance. (The information was obtained from private communication with Oliver Gutsche.) An alternative estimate is based on the cost of renting the CPU time from industry, at a rate of 1.4 US dollar cents per core hour. (The information was obtained from private communication with Oliver Gutsche.) The 0.9 (1.4) US dollar cents assumption puts the annual cost of simulation for CMS in the range of 3.5-6.2 (5.5-10) million US dollars, half of it spent on executing the Geant4 module. A corollary to this discussion is that improvements of $1 \%, 10 \%$, and $35 \%$ in the time performance of the Geant 4 toolkit would render $50-80 \mathrm{k}, 500-800 \mathrm{k}, 1.8-2.8 \mathrm{M}$ US dollars per year of savings to CMS. Improvements on the order of a 2-5 speed-up factor, as targeted by current R\&D efforts (GeantV [78]), would yield savings on the order of 2-4 (3-6) million US 
dollars per year. An important question, rarely addressed even by modern experiments at the time of detector design and technology selection, is related to the added costs to the detector construction, commissioning, and operations that comes from detector choices that maximize physics output in exchange for expensive and time-consuming simulation and reconstruction operations.

It is important to mention that the LHC experiments expect their computing needs to increase by a factor of 10 to 100 in the High-Luminosity LHC (HL-LHC) era, depending on the solutions developed to face simulation, pile-up, and reconstruction challenges arising from the high-luminosity environment. In principle, reconstruction would take a larger fraction of the computing resources during the HL-LHC era, since the CPU time consumption is predicted to increase exponentially with the number of pile-up events. However, while simulation code is highly optimized and offers few non-revolutionary time performance improvement opportunities, the reconstruction code under development for the upgraded or new HL-LHC sub-detector systems still offers low hanging fruit to exploit, at least in the case of CMS. Consequently, a significant improvement in simulation computing performance is a need in present times of flat budgets, and so are the research efforts with that goal in mind.

The Geant4 Collaboration has gone to great lengths to improve the toolkit computing performance during the last few years, as code was reviewed and optimized. In 2013, the introduction of event-level multithreading capabilities in Geant4 brought significant memory savings, as illustrated in Fig. 55, which shows the CPU time (top) and memory (bottom) consumption ratios of a CMS standalone simulation application (outside of the CMS software framework), based on Geant4 version 10.1.p02, executed for $5 \mathrm{GeV}$ electrons in multithreaded and sequential modes. While time performance does not improve, deviating from perfect scaling by approximately $10 \%$ when executed on 30 cores, memory consumption improves significantly with $170 \mathrm{MB}$ used in the first event, and only $30 \mathrm{MB}$ per event used by each additional thread 79 .

Figs. 56 57 show the percentage change in CPU time performance taking Geant4 version 10.0 as a reference, starting with Geant4 version 9.4.p02 (2010) and ending with Geant4 version 10.2 (2015) for the standalone CMS application and a simple calorimeter configuration made of $\mathrm{Cu}$-Scintillator in a 4 Tesla magnetic field [79]. The study is performed for $50 \mathrm{GeV} e^{-}, \pi^{-}$, and protons, as well as for $H \rightarrow Z Z$ events. In average, the time performance improvement through the life of the LHC experiments (2010-2015) is of the order of $35 \%$. All tests were performed on the same hardware (AMC Opteron $6128 \mathrm{HE} @ 2 \mathrm{GHz}$ ) using the same operating system. Remarkably, the percentage time performance improvement during the period of time shown in the plots is in the double digits, even as the physics models were improved significantly for accuracy, something that typically comes associated with a time performance penalty.

The cost of simulation presented before refers only to the purchase, life-cycle, operations, and maintenance of the computing resources allocated to the task. The values do not include the design, development, validation, operation, and support of the simulation tool-kits, such as Geant4, or of the simulation software in the experiments. As a reference, during its 22 years of existence, the person- 

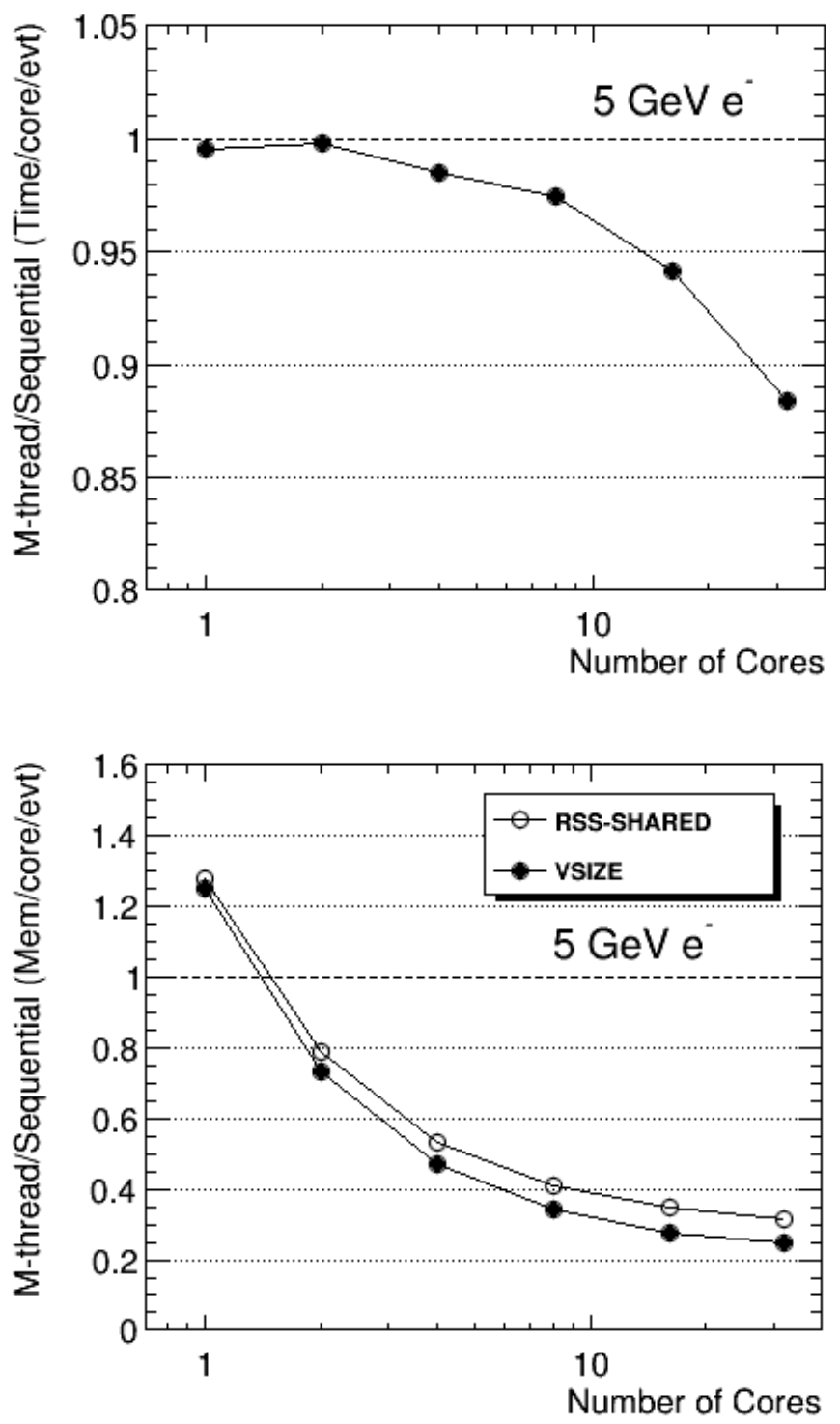

Figure 55: CPU time (top) and memory (bottom) ratios for a CMS standalone simulation application (outside of the CMS software framework) based on Geant4 version 10.1.p02 executed for $5 \mathrm{GeV}$ electrons in multithreaded and sequential modes 79 . 


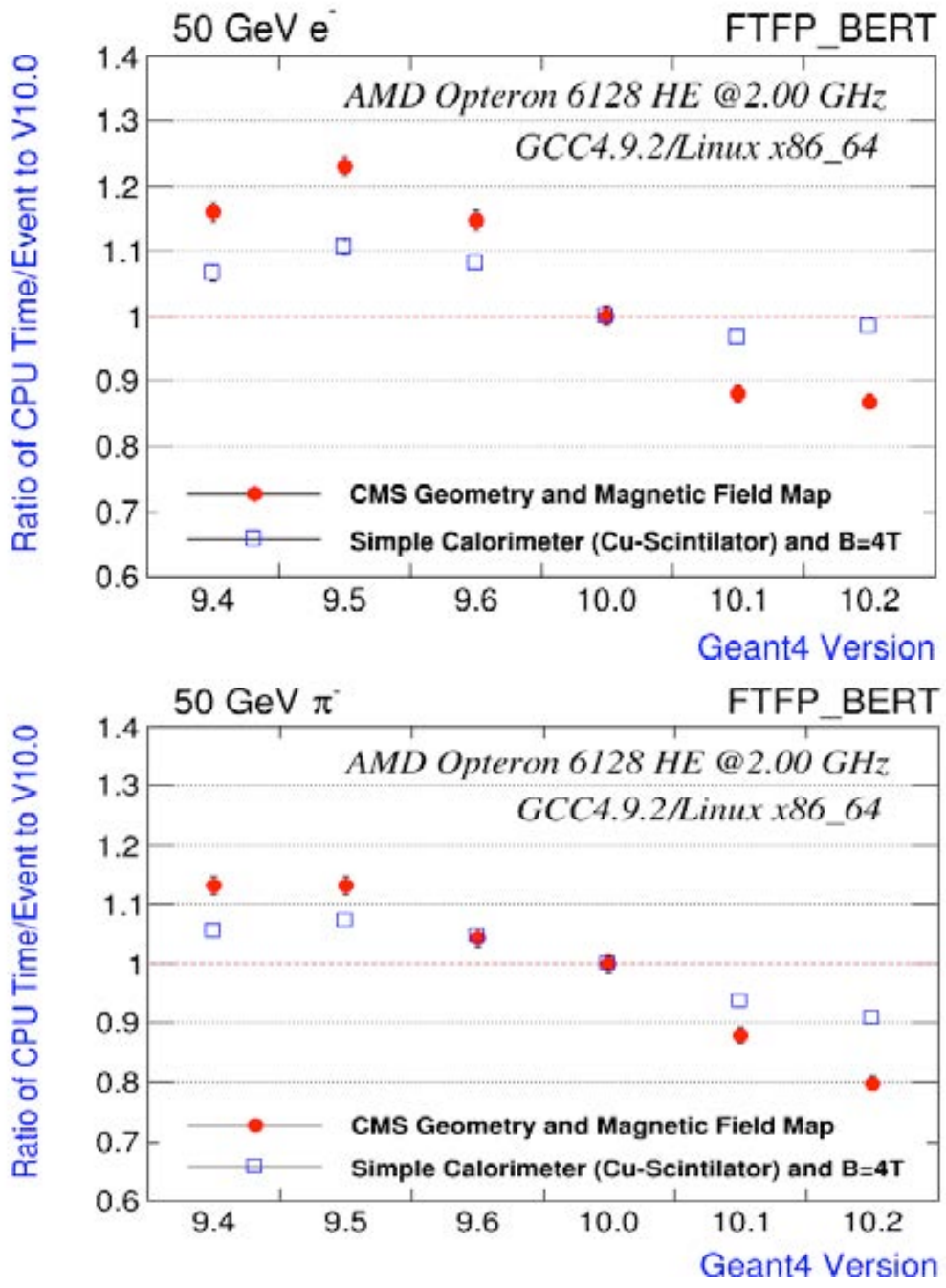

Figure 56: Percentage change in CPU time performance with respect to Geant4 version 10.0, starting with Geant4 version 9.4.p02 (2010) and ending with Geant4 version 10.2 (2015), for a standalone CMS application (outside of the CMS software framework) and a simple calorimeter configuration made of $\mathrm{Cu}-\mathrm{Scintillator}$ in a 4 Tesla magnetic field. The study is performed for $50 \mathrm{GeV} e^{-}$and $\pi^{-} 79$. 

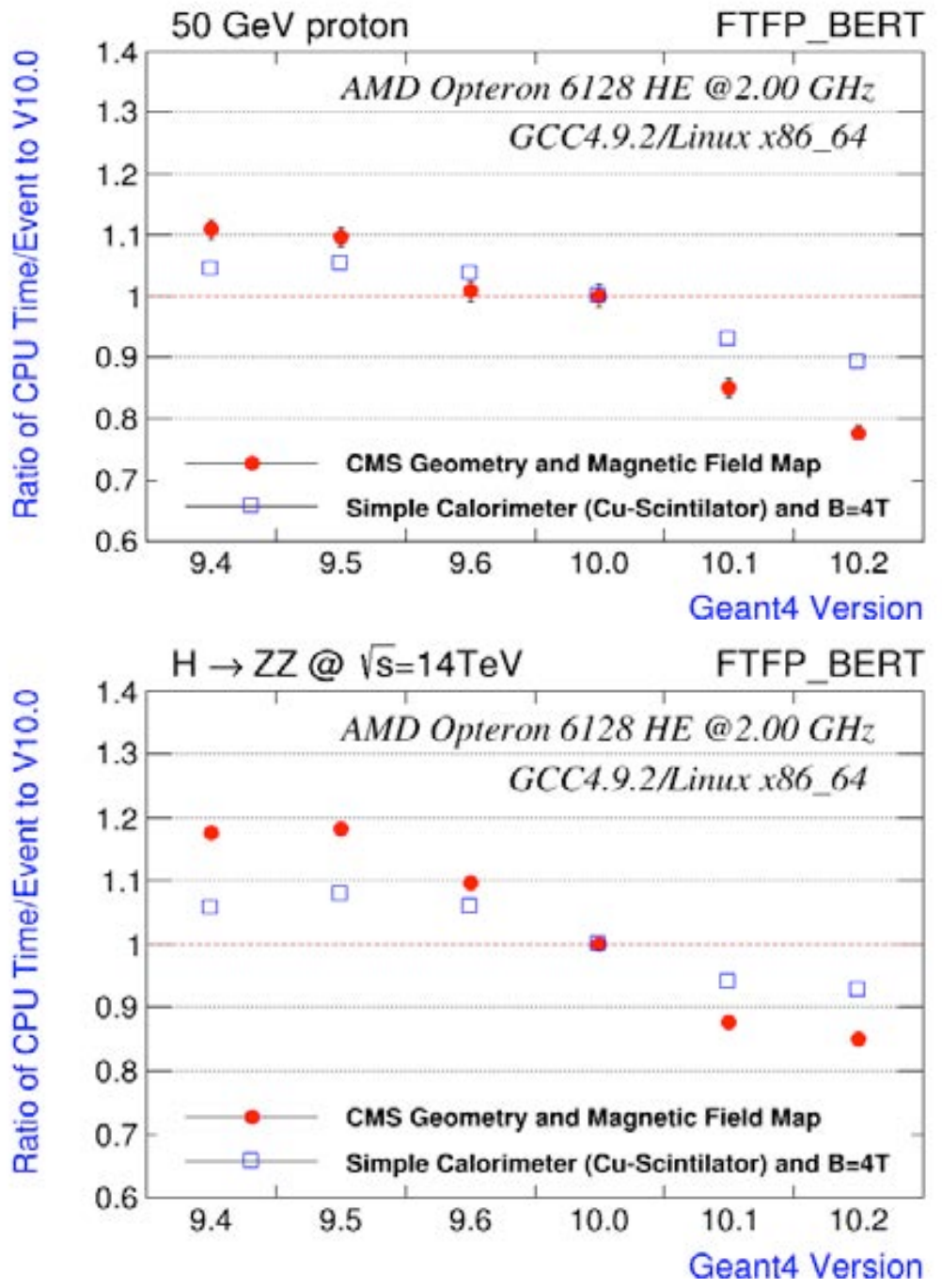

Figure 57: Percentage change in CPU time performance with respect to Geant4 version 10.0, starting with Geant4 version 9.4.p02 (2010) and ending with Geant4 version 10.2 (2015), for a standalone CMS application (outside of the CMS software framework) and a simple calorimeter configuration made of $\mathrm{Cu}-\mathrm{Scintillator}$ in a 4 Tesla magnetic field. The study is performed for protons and $H \rightarrow Z Z$ events [79]. 
power investment in Geant 4 has totaled more than 500 person-years, equivalent to about $100 \mathrm{M}$ US dollars, including fringe benefits and overhead. Additional investments of the same order have been made by the major $21^{\text {st }}$ century HEP experiments on detector specific simulation and reconstruction software. An interesting corollary is that the cost of the physics software amounts to a significant fraction of the cost of the detectors.

It would be an interesting exercise to estimate the cost of running a modern HEP experiment with and without efficient simulation tool-kits and full simulation software. The truth is that the experiments as we know them today would simply not exist without these tools. How much physics would be lost to a deficient detector design or poor optimization? How would the design and operation of systems such as data acquisition, distribution, storage, and analysis workflows be affected? How accurate, efficient, and fast would reconstruction algorithms, calibration and analysis methods be? How much person-power and how many years of delay in delivering scientific publications would it cost to reproduce, without good quality simulation, the level of accuracy in physics measurements achieved by modern experiments? Is it possible at all to deliver physics of the quality and accuracy we produce today without simulation?

\section{The Future}

The accuracy of the simulation software developed for the current generation of high-energy physics detectors, coupled with the speed of contemporary computers, has enabled the experiments to perform tasks that scientists could only have dreamed of before. Simulation helps physicists to design and optimize detectors for best physics performance, stress-test the computing infrastructure, program data reconstruction algorithms that perform almost as in design specifications at the begining of the experiment run, develop data-driven techniques for calibration and physics analysis, and produce data samples with the properties predicted by many candidate theories to describe currently unexplained physical phenomena.

Modern HEP experiments generate and handle an enormous amount of real and simulated data. For its size and complexity, these data has earned a place in the world of what is known as Big Data. The experiments at the CERN Large Hadron Collider (LHC) have produced, reconstructed, stored, transferred, and analyzed tens of billion of simulated events during the first two runs. According to Ref. [80, the amount of data collected and stored by the LHC experiments through the end of 2013 was of the order of $15 \mathrm{~PB} /$ year, not so far from the $180 \mathrm{~PB} /$ year uploaded to Facebook, the $98 \mathrm{~PB}$ of data in the Google search index, or the $15 \mathrm{~PB} /$ year in videos uploaded to YouTube. Integrated on time over the last two decades, the cost of simulation and reconstruction in large modern HEP experiments exceeded the one hundred million dollars mark.

The high instantaneous luminosity required at the LHC experiments, needed to reach the $3000 \mathrm{fb}^{-1}$ integrated luminosity milestone associated with the highluminosity LHC physics program, will tax heavily the performance of the reconstruction algorithms. Through the end of the 2030's, the experiments expect 
to collect 150 times more data than in Run 1 . The $50 \mathrm{~PB}$ of raw data produced in 2016 will grow to approximately 600 PB in 2026 while the CPU needs will increase by a factor of about 60 . The exact numbers will depend on the approximations and the loss of information that the experiments are willing to tolerate to keep computing performance within the limits established by the available resources. Thus, the effort to improve the computing performance of the simulation and reconstruction software requires immediate attention, in order to restrain the increasing demand of computing power within the limits of flat budgets. Although transistor density growth is more or less keeping up with Moore's law, doubling every couple of years, clock speed has been flat since approximately 2003. Consequently, solutions must be found elsewhere, leveraging the core count growth in multicore machines, using new generation coprocessors, and re-engineering code under new programming paradigms based on concurrency and parallel programming. Coprocessors, or accelerators, specialize in operations such as floating point math or graphics. A hybrid computing model would allow to share work across a mixture of computers with different architectures. Each processor type could be used to perform different tasks depending on its nature.

In parallel, experiments are transforming their software frameworks to support event multithreading and task-level parallelization. In the specific case of Geant4, the release of the first version with multithreading capability in 2013 allowed significant savings in memory, although not in time performance. For the latter, expert teams are invested in $\mathrm{R} \& \mathrm{D}$ programs to explore the potential of multithread track-level (particle-level) parallelization, improved instruction pipelining, data locality, and vectorization for single instruction multiple data. One example is the GeantV [78] project to develop the next generation detector simulation toolkit, with a goal set to achieve a speedup factor of 2 to 5 with respect to Geant4, while enhancing the physics accuracy of the code and offering fast simulation options that include machine learning techniques for fast and precise tuning.

Breakthroughs in the design of simulation and reconstruction code, exploiting the benefits of fine granularity parallelism in applications running in modern computer architectures, will be essential to address the software and computing challenges faced by the HEP experiments of the 21st century.

\section{Acknowledgements}

Funding: Fermilab is operated by Fermi Research Alliance, LLC under Contract No. DE-AC02-07CH11359 with the U.S. Department of Energy.

I would like to express my gratitude to the many people I have consulted on the material to include in this review. First and foremost, I am eternally grateful to John Harvey, former leader of the software group at CERN, who initially asked me a few questions about the impact of simulation in experimental particle physics, eventually encouraged me to write this article, and was the first person to read and comment on a draft. I am also grateful to my Geant4 co-collaborators who built on previous Geant experience and developed 
the most widespread and successful detector simulation toolkit the HEP field has ever known. Their work has yet to receive the recognition and support it deserves. I want to thank Federico Carminati, Guenther Dissertori, and Paris Sphicas for reading and commenting on a draft. Thanks to my ATLAS colleagues John Chapman, Andrea Dotti, Zach Marshall, and Ariel Schwartzman for pointing me to outstanding ATLAS material on simulation, test beams, jets, and missing transverse energy. In order of topic appearance in the paper, I would like to thank Julia Yarba and Alberto Ribon for pointing me to specific Geant4 physics validation plots and associated information, Sunanda Banerjee for useful discussions and the figures with the CMS test beam and single particle response comparisons, Mike Tartaglia and Harrison Prosper for trying hard to recall details of the D0 test beam experiments, my many D0 colleagues with whom we navigated the difficult waters of producing high quality physics measurements with neither a solenoidal field nor fast-enough high-quality simulation software during Run 1, Liz Sexton-Kennedy and Robert Harris for discussions on the CDF simulation software and first inclusive jet cross section results, Soon Young Jun for providing precise information and documentation on the CDF Monte Carlo tuning effort and for the Geant4 computing performance plots, Marjorie Shapiro for private communication on the CDF QFL fast simulation software, my CMS colleagues with whom we experienced the thrill of developing outstanding simulation code and producing the most precise physics measurements ever in a hadron collider, Kevin Burkett for pointing me to CDF publications data statistics, Oliver Gutsche for providing most of the information for the cost evaluation of the simulation operation in CMS. Last but not least, I want to thank Krzysztof Genser and the rest of the members of my Fermilab Physics and Detector Simulation group (PDS) for their hard work in the area of simulation software research, development and support, as well as the Fermilab Scientific Computing Division and the US Department of Energy for their continued support of the Fermilab Geant operations and research programs.

\section{References}

[1] S. Agostineli et al. (Geant4 Collaboration), Gean4-a simulation toolkit, Nucl. Instrum. Meth. A 506 (2003) 250-303. doi:10.1016/ S0168-9002(03) 01368-8.

[2] J. Allison et al. (Geant4 Collaboration), Recent developments in Geant4, Nucl. Instrum. Meth. A 835 (2016) 186-225. doi:10.1016/j.nima.2016. 06.125

[3] R. Ford, W. Nelson, The EGS Code System - Version 3, Stanford Linear Accelerator Center Report SLAC-210.

[4] R. Brun, F. Bruyant, M. Maire, A. McPherson, P. Zanarini, Geant3 CERNDD-EE-84-1. 
[5] The ATLAS Collaboration, Observation of a new particle in the search for the Standard Model Higgs boson with the ATLAS detector at the LHC, Phys. Lett. B 716 (2012) 1-29. doi:10.1016/j.physletb.2012.08.020.

[6] The CMS Collaboration, Observation of a new boson at a mass of $125 \mathrm{GeV}$ with the CMS experiment at the LHC, Phys. Lett. B 716 (2012) 30-61. doi:10.1016/j.physletb.2012.08.021.

[7] D.J. Lange et al. for the CMS Collaboration, Upgrades for the CMS full simulation, J. Phys. Conf. Ser. 608 (2015) 012056.

[8] S. Banerjee et al. for the CMS Collaboration, Validation and tuning of the CMS simulation, J. Phys. Conf. Ser. 331 (2011) 032015.

[9] V. Daniel Elvira for the CMS Collaboration, Readiness of the CMS detector simulation, NSS/MIC 2007 C07-10-28 (2007) 2081-2085. doi:10.1109/ NSSMIC.2007.4436563.

[10] P. J. Clark for the ATLAS Collaboration, The ATLAS Detector Simulation, Nucl. Phys. B - Proceedings Supplements 215 (2011) 85-88. doi:10.1016/ j.nuclphysbps.2011.03.142.

[11] Z. Marshall for the ATLAS Collaboration, Validation and performance studies for the ATLAS simulation, J. Phys. Conf. Ser. 219 (2010) 032016.

[12] A. Rimoldi for the ATLAS Collaboration, The ATLAS detector simulation application, Nucl. Phys. B - Proceedings Supplements 172 (2007) 49-52. doi:10.1016/j.nuclphysbps.2007.07.005

[13] G. Grindhammer et al., The Fast Simulation of Electromagnetic and Hadronic Showers, Nucl. Instrum. Meth. A 290 (1990) 469.

[14] A. Ferrari, P. Sala, A. Fasso, , J. Ranft, FLUKA: a multi-particle transport code, CERN-2005-10 (2005), INFN/TC_05/11, SLAC-R-773.

[15] The MARS Code System http://mars.fnal.gov.

[16] C. Adloff et al., Study of the interactions of pions in the CALICE silicontungsten calorimeter prototype, JINST 5 (2010) 05007. doi:10.1088/ 1748-0221/5/05/P05007.

[17] M. Apollonio et al. (HARP Collaboration), Forward production of charged pions with incident $\pi^{+} /-$on nuclear targets measured at the CERN PS, Nucl. Phys. A 821 (2009) 118-192. doi:10.1016/j.nuclphysa.2009.01. 080.

[18] M. Apollonio et al. (HARP Collaboration), Large-angle production of charged pions with incident pion beams on nuclear targets, Phys. Rev. C 80 (2009) 065207. doi:10.1103/PhysRevC.80.065207. 
[19] M. G. Catanesi et al. (HARP Collaboration), Large-angle production of charged pions with 3-12 GeV/c incident protons on nuclear targets, Phys. Rev. C 77 (2008) 055207. doi:10.1103/PhysRevC.77.055207.

[20] C. Alt et al. (NA49 Collaboration), Inclusive production of charged pions in p+C collisions at $158 \mathrm{GeV} / \mathrm{c}$ beam momentum, Eur. Phys. J. C 49 (2007) 897-917. doi:10.1140/epjc/s10052-006-0165-7.

[21] C. Alt et al. (NA49 Collaboration), Inclusive production of charged pions in $\mathrm{p}+\mathrm{p}$ collisions at $158 \mathrm{GeV} / \mathrm{c}$ beam momentum, Eur. Phys. J. C 45 (2006) 343-381. doi:10.1140/epjc/s2005-02391-9.

[22] T. Anticic et al. (NA49 Collaboration), Inclusive production of protons, anti-protons and neutrons in $\mathrm{p}+\mathrm{p}$ collisions at $158 \mathrm{GeV} / \mathrm{c}$ beam momentum, Eur. Phys. J. C 65 (2010) 9-63. doi:10.1140/epjc/s10052-009-1172-2

[23] N. Abgrall et al. (NA61/SHINE Collaboration), Measurement of production properties of positively charged kaons in proton-carbon interactions at $31 \mathrm{GeV} / \mathrm{c}$, Phys. Rev. C 85 (2012) 035210. doi:10.1103/PhysRevC.85. 035210

[24] N. Abgrall et al. (NA61/SHINE Collaboration), Measurements of cross sections and charged pion spectra in proton-carbon interactions at $31 \mathrm{GeV} / \mathrm{c}$, Phys. Rev. C 84 (2011) 034604. doi:10.1103/PhysRevC.84.034604

[25] V.V. Uzhinsky for the Geant4 Hadronic Working Group, The Fritiof (FTF) Model in Geant4, International Conference on Calorimetry for the High Energy Frontier (CHEF 2013) C13-04-22.4 (2013) 260-264.

[26] D. Wright, M. Kelsey, The Geant4 Bertini Cascade, Nucl. Instrum. Meth. A 804 (2015) 175-188. doi:10.1016/j.nima.2015.09.058.

[27] Yu. D. Bayukov et al., Angular Dependences Of Inclusive Nucleon Production In Nuclear Reactions At High-energies And Separation Of Contributions From Quasifree And Deep Inelastic Nuclear Processes, Sov. J. Nucl. Phys. 42 (1985) 116.

[28] S. Banerjee for the CMS Collaboration, Validation of Physics Models of Geant4 using data from CMS experiment, To be published in Journal of Physics Conference Series (JPCS). Proceedings of the CHEP 2016 Conference, San Francisco https://twiki.cern.ch/twiki/bin/viewauth/CMS/Geant4Validation2016.

[29] The ATLAS Collaboration, A measurement of the calorimeter response to single hadrons and determination of the jet energy scale uncertainty using LHC Run-1 pp-collision data with the ATLAS detector, Eur. Phys. J. C (2017) 77:26. doi:10.1140/epjc/s10052-016-4580-0.

[30] ATLAS Public Results https://twiki.cern.ch/twiki/bin/view/AtlasPublic/ ApprovedPlotsTileTestBeamResults. 
[31] The D0 Collaboration, Beam tests of the D0 uranium liquid argon end calorimeters, Nucl. Instrum. Meth. A 324 (1993) 53-76. doi:10.1016/ 0168-9002(93) 90965-K.

[32] The D0 Collaboration, Jet energy scale determination in the D0 experiment, Nucl. Instrum. Meth. A 763 (2014) 442-475. doi:10.1016/j.nima. 2014.05 .044

[33] The D0 Collaboration, Determination of the Absolute Jet Energy Scale in the DZERO Calorimeters, Nucl. Instrum. Meth. A 424 (1999) 352-394. doi:10.1016/S0168-9002(98)01368-0

[34] A. Bhatti et al., Determination of the jet energy scale at the collider detector at Fermilab, Nucl. Instum. Meth. A 566 (2006) 375-412. doi: 10.1016/j.nima.2006.05.269.

[35] The CMS Collaboration, Determination of Jet Energy Calibration and Transverse Momentum Resolution in CMS, JINST 6 (2011) 11002. doi: 10.1088/1748-0221/6/11/P11002.

[36] The ATLAS Collaboration, Jet energy measurement with the ATLAS detector in proton-proton collisions at $\sqrt{s}=7 \mathrm{TeV}$, Eur. Phys. J. C 73 (2013) 2304. doi:10.1140/epjc/s10052-013-2304-2.

[37] Florian Beaudette for the CMS Collaboration, The CMS Particle Flow Algorithm, International Conference on Calorimetry for the High Energy Frontier (CHEF 2013) C13-04-22.4 (2013) 295-304.

[38] The ATLAS Collaboration, Jet energy measurement and its systematic uncertainty in proton-proton collisions at $\sqrt{s}=7 \mathrm{TeV}$ with the ATLAS detector, Eur. Phys. J. C 75 (2015) 17. doi:10.1140/epjc/ s10052-014-3190-y.

[39] The ATLAS Collaboration, Jet energy resolution in proton-proton collisions at $\sqrt{s}=7 \mathrm{TeV}$ recorded in 2010 with the ATLAS detector, Eur. Phys. J. C 73 (2013) 2306. doi:10.1140/epjc/s10052-013-2306-0.

[40] S. P. Martin, A Supersymmetry Primer arXiv:hep-ph/9709356.

[41] The CMS Collaboration, Search for New Physics with Jets and Missing Transverse Momentum in pp collisions at $\sqrt{s}=7 \mathrm{TeV}$, JHEP 8 (2011) 155. doi:10.1007/JHEP08(2011)155.

[42] T. Schum, QCD Processes and Search for Supersymmetry at the LHC, DESY-THESIS-2012-029.

[43] The CMS Collaboration, Performance of CMS muon reconstruction in pp collision events at $\sqrt{s}=7 \mathrm{TeV}$, JINST 7 (2012) 10002. doi:10.1088/ 1748-0221/7/10/P10002. 
[44] The CMS Collaboration, Performance of electron reconstruction and selection with the CMS detector in proton-proton collisions at $\sqrt{s}=8 \mathrm{TeV}$, JINST 10 (2015) 06005. doi:10.1088/1748-0221/10/06/P06005.

[45] The ATLAS Collaboration, Measurement of the muon reconstruction performance of the ATLAS detector using 2011 and 2012 LHC proton-proton collision data, Eur. Phys. J. C 74 (2014) 3130. doi:10.1140/epjc/ s10052-014-3130-x.

[46] The ATLAS Collaboration, Electron efficiency measurements with the ATLAS detector using 2012 LHC proton-proton collision data, Submitted to Eur. Phys. J. C arXiv:1612.01456 [hep-ex].

[47] The CMS Collaboration, Search for new physics in the multijet and missing transverse momentum final state in proton-proton collisions at $\sqrt{s}=8 \mathrm{TeV}$, JHEP 6 (2014) 055. doi:10.1007/JHEP06 (2014) 055

[48] The CMS Collaboration, CMS Phase II Upgrade Scope Document, CERNLHCC-2015-019 https://cds.cern.ch/record/2055167.

[49] J.de Favereau et al., DELPHES 3: a modular framework for fast simulation of a generic collider experiment, JHEP 02 (2014) 057. doi: 10.1007/JHEP02(2014)057.

[50] The ATLAS Collaboration, ATLAS Phase II Upgrade Scope Document, CERN-LHCC-2015-020 https://cds.cern.ch/record/2055248.

[51] WLCG: Worldwide LHC Computing Grid, http://wlcgpublic.web.cern.ch/tier-centres.

[52] D. Futyan, R. Mankel, Christoph Paus for the CMS Collaboration, The CMS Computing, Software and Analysis Challenge, Journal of Physics: Conference Series 219 (2010) 032008. doi:10.1088/1742-6596/219/3/ 032008

[53] The CMS Collaboration, Description and performance of track and primary-vertex reconstruction with the CMS tracker, JINST 9 (2014) 10009. doi:10.1088/1748-0221/9/10/P10009.

[54] The CMS Collaboration, Studies of Tracker Material, CMS Public Note, CMS-PAS-TRK-10-003 https://cds.cern.ch/record/1279138.

[55] The ATLAS Collaboration, Photon Conversions at $\sqrt{s}=900 \mathrm{GeV}$ measured with the ATLAS Detector, ATLAS Conference Note, ATLAS-CONF2010-007 https://cds.cern.ch/record/1274001.

[56] The ATLAS Collaboration, Study of the Material Budget in the ATLAS Inner Detector with $K_{S}^{0}$ decays in collision data at $\sqrt{s}=900 \mathrm{GeV}$, ATLAS Conference Note, ATLAS-CONF-2010-019 https://cds.cern.ch/record/1277651. 
[57] The CMS Collaboration, Identification of b-quark jets with the CMS experiment, JINST 8 (2013) 04013. doi:10.1088/1748-0221/8/04/P04013

[58] The ATLAS Collaboration, Performance of b-jet identification in the ATLAS experiment, JINST 11 (2016) 04008. doi:10.1088/1748-0221/11/ 04/P04008.

[59] Torbjorn Sjostrand et al., An Introduction to PYTHIA 8.2, arXiv:1410.3012 [hep-ph doi:10.1016/j.cpc.2015.01.024.

[60] T. Sjostrand, S. Mrenna, P. Skands, PYTHIA 6.4 Physics and Manual, JHEP05 (2006) 026doi:10.1088/1126-6708/2006/05/026.

[61] J. Alwall, R. Frederix, S. Frixione, V. Hirschi, F. Maltoni, O. Mattelaer, H.-S. Shao, T. Stelzer, P. Torrielli, M. Zaro, The automated computation of tree-level and next-to-leading order differential cross sections, and their matching to parton shower simulations, JHEP07 (2014) 079 doi:10.1007/ JHEP07 (2014)079.

[62] The CMS Collaboration, Performance of the CMS missing transverse energy reconstruction in $p p$ data at $\sqrt{s}=8 \mathrm{TeV}$, JINST 10 (2015) 2006. doi:10.1088/1748-0221/10/02/P02006.

[63] The ATLAS Collaboration, Measurement of $W^{ \pm}$and $Z$-boson production cross sections in pp collisions at $\sqrt{s}=13 \mathrm{TeV}$ with the ATLAS detector, Phys. Lett. B 759 (2016) 601. doi:10.1016/j.physletb.2016.06.023.

[64] The ATLAS Collaboration, Performance of algorithms that reconstruct missing transverse momentum in $\sqrt{s}=8 \mathrm{TeV}$ protonproton collisions in the ATLAS detector, Submitted to Eur. Phys. J. C arXiv:1609.09324 [hep$\mathrm{ex}]$.

[65] The ATLAS Collaboration, Measurement of inclusive jet and dijet production in $p p$ collisions at $\sqrt{s}=7 \mathrm{TeV}$ using the ATLAS detector, Phys. Rev. D 86 (2012) 014022. doi:10.1103/PhysRevD.86.014022.

[66] The CMS Collaboration, Measurement of the Inclusive Jet Cross Section in $p p$ Collisions at $\sqrt{s}=7 \mathrm{TeV}$, Phys. Rev. Lett. 107 (2011) 132001. doi: 10.1103/PhysRevLett.107.132001

[67] The CDF Collaboration, Inclusive Jet Cross Section in $\bar{p} p$ Collisions at $\sqrt{s}=1.8 \mathrm{TeV}$, Phys. Rev. Lett. 77 (1996) 438. doi:10.1103/ PhysRevLett.77.438

[68] The D0 Collaboration, Inclusive Jet Cross Section in $\bar{p} p$ Collisions at $\sqrt{s}=$ 1.8 TeV, Phys. Rev. Lett. 82 (1999) 2451. doi:10.1103/PhysRevLett. 82. 2451 . 
[69] The ATLAS Collaboration, Measurement of inclusive jet and dijet cross sections in proton-proton collisions at $7 \mathrm{TeV}$ centre-of-mass energy with the ATLAS detector, Eur. Phys. J. C 71 (2011) 1512. doi:10.1140/epjc/ s10052-010-1512-2.

[70] The D0 Collaboration, Inclusive Jet Production in $\bar{p} p$ Collisions, Phys. Rev. Lett. 86 (2001) 1707-1712. doi:10.1103/PhysRevLett.86.1707.

[71] The CDF Collaboration, Measurement of the inclusive jet cross section at the Fermilab Tevatron $p \bar{p}$ collider using a cone-based jet algorithm, Phys. Rev. D 78 (2008) 052006. doi:10.1103/PhysRevD.78.052006.

[72] The D0 Collaboration, Measurement of the inclusive jet cross section in $p \bar{p}$ collisions at $\sqrt{s}=1.96 \mathrm{TeV}$, Phys. Rev. D 85 (2012) 052006. doi: 10.1103/PhysRevD.85.052006.

[73] The CDF Collaboration, Measurement of the Inclusive Jet Cross Section in $\bar{p} p$ Collisions at $\sqrt{s}=1.8 \mathrm{TeV}$, Phys. Rev. Lett. 62 (1989) 613. doi: 10.1103/PhysRevLett.62.613.

[74] The CDF Collaboration, Inclusive jet cross section in $\bar{p} p$ collisions at $\sqrt{s}=$ 1.8 TeV, Phys. Rev. Lett. 68 (1992) 1104. doi:10.1103/PhysRevLett. 68. 1104 .

[75] Summary Table https://www-cdf.fnal.gov/physics/physics.html.

[76] Year-to-year history of paper submissions https://wwwd0.fnal.gov/Run2Physics/WWW/results.htm.

[77] CMS Publications versus Time http://cms-results.web.cern.ch/cmsresults/public-results/publications-vs-time/.

[78] J. Apostolakis et al. (GeantV Development Group), Adaptive track scheduling to optimize concurrency and vectorization in GeantV, J. Phys.: Conf. Ser. 608 (2015) 012003. doi:10.1088/1742-6596/608/1/012003.

[79] Source: Geant4 Testing and Quality Assurance Working Group https://g4cpt.fnal.gov/g4p/prplots/cpu_by_version.html.

[80] J. Pearlstein, Information Revolution: Big Data Has Arrived at an Almost Unimaginable Scale, Wired Magazine https://www.wired.com/2013/04/bigdata. 